\title{
Multi-pulse SCR Rectifiers
}

by

\section{Mei Li}

\author{
A project \\ presented to Ryerson University \\ in partial fulfillment of the \\ requirements for the degree of \\ Master of Engineering \\ in the Program of
}

Electrical and Computer Engineering

Toronto, Ontario, Canada, 2005

(C) Mei Li 2005 


\section{UMI Number: EC53041}

\section{All rights reserved \\ INFORMATION TO USERS}

The quality of this reproduction is dependent upon the quality of the copy submitted. Broken or indistinct print, colored or poor quality illustrations and photographs, print bleed-through, substandard margins, and improper alignment can adversely affect reproduction.

In the unlikely event that the author did not send a complete manuscript and there are missing pages, these will be noted. Also, if unauthorized copyright material had to be removed, a note will indicate the deletion.

\section{$\mathrm{UMI}^{\circ}$}

UMI Microform EC53041

Copyright 2008 by ProQuest LLC

All rights reserved. This microform edition is protected against unauthorized copying under Title 17, United States Code.

ProQuest LLC

789 East Eisenhower Parkway

P.O. Box 1346

Ann Arbor, MI 48106-1346 



\section{Instructions on Borrower's}

Ryerson Universiry requires the signatures of all persons using or photocopying this sis. Please sign below, and give address and date. 


\section{Abstract}

This thesis presents the introduction, analysis and experimental verification of the sixpulse SCR rectifier and multi-pulse SCR rectifiers.

As a fundamental three-phase controllable ac-dc converter, the six-pulse SCR rectifier is widely used in industry. However, it generates high Total Harmonic Distortion (THD) in the line current. One of the solutions is to use multi-pulse rectifiers. Multi-pulse rectifiers could be classified into the 12-, 18- and 24-pulse configurations. Application examples include high voltage direct current transmission systems, high power battery chargers and load commutated current source inverter powered motor drives.

In this thesis, the six-, 12-, 18- and 24-pulse SCR rectifiers with inductive and capacitive loads are introduced. The line current THD and the input PF of various rectifiers are investigated. The principle of the harmonic elimination through phaseshifting transforms is analyzed by Fourier analysis and positive/negative sequence analysis. The experimental verification is accomplished on a prototype of the 12-pulse $\mathrm{SCR}$ rectifier.

Key words: multi-pulse SCR rectifier, harmonic elimination, line current THD. 


\section{Acknowledgements}

A sincere appreciation is expressing to my supervisor, Professor $\mathrm{Bin} \mathrm{Wu}$, for his found guidance in my graduate study.

Thanks also go to all professors in the power field and research personnel in the soratory for Electric Drive Research and Applications (LEDAR) at Ryerson University, who offered valuable help on my study.

Encouragement from my family is important as well. 


\section{Table of Contents}

Author's Declaration ii

Instructions on Borrower's iii

Abstract iv

Acknowledgements $\quad$ v

Table of Contents vi

List of Figures ix

List of Acronyms _ xi

Chapter 1 Introduction $\quad 1$

1.1 Introduction of the Six-pulse SCR Rectifier ............................. 3

1.2 Introduction of Multi-pulse SCR Rectifiers ............................... 4

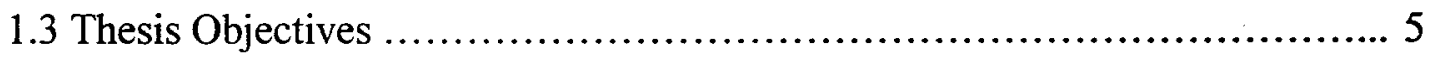

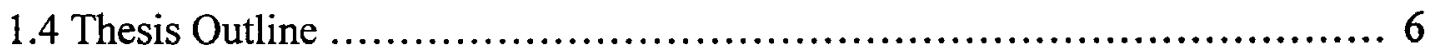

$\begin{array}{ll}\text { Chapter } 2 \text { Six-pulse SCR Rectifier } & 7\end{array}$

2.1 Six-Pulse SCR Rectifier with Inductive Loads ........................ 7

2.1.1 Circuit Configuration.............................................. 7

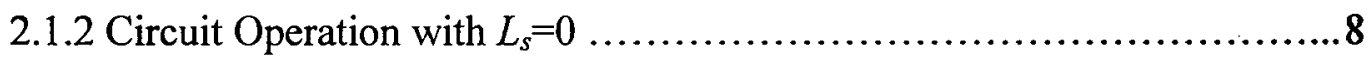

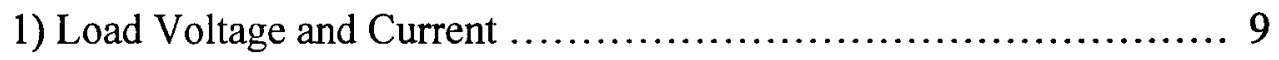

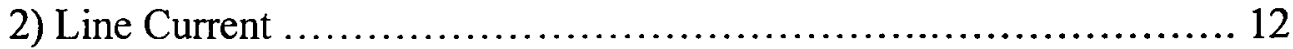

3) Input PF of the SCR Rectifier ................................ 13

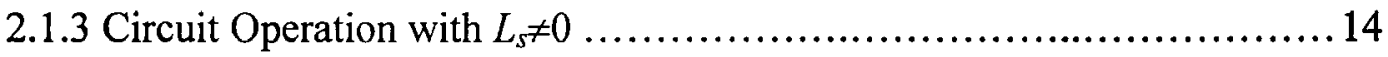

1) Commutation Interval and Corrected Load Voltage and Current ....... 14

2) The Line Current THD and the Input PF ..................... 16

2.2 Six-Pulse SCR Rectifier with Capacitive Loads ......................... 17

2.2.1 Circuit Configuration ............................................ 17

2.2.2 Current Waveforms .............................................. 18 
2.2.3 The Line Current THD and the Input PF

Summary

apter 3 12-Pulse SCR Rectifier $\quad 21$

12-Pulse SCR Rectifier with Inductive Loads .......................... 21

3.1.1 Circuit Configuration ...........................................21

3.1.2 Voltage and Current ............................................... 23

1) Voltage and Current Waveforms ................................ 23

2) Average Load Voltage ........................................... 24

3) RMS of the Transformer Primary Side Line Current ....................25

3.1.3 Analysis of Harmonic Elimination ................................ 25

1) Fourier Analysis ............................................... 25

2) Positive/Negative Sequence Analysis ............................ 28

3.1.4 The Line Current THD and the Input PF .......................... 31

3.2 12-Pulse SCR Rectifier with the Capacitive Load .......................... 32

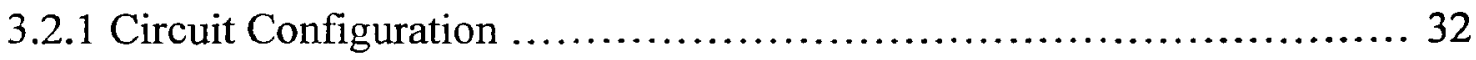

3.2.2 Current Waveforms .............................................. 33

3.2.3 The Line Current THD and the Input PF ......................... 34

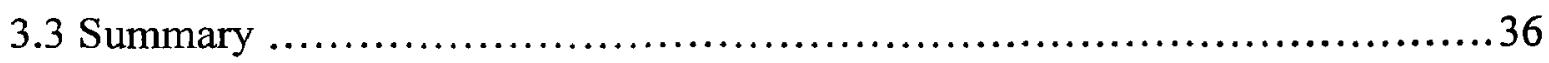

Chapter 4 18-Pulse SCR Rectifier $\quad 37$

4.1 18-Pulse SCR Rectifier with Inductive Loads .......................... 37

4.1.1 Circuit Configuration ........................................... 37

4.1.2 Voltage and Current Waveforms ................................... 38

4.1.3 Analysis of Harmonic Elimination .................................. 40

4.1.4 The Line Current THD and the Input PF ..........................40

4.2 18-Pulse SCR Rectifier with the Capacitive Load ..............................41

4.2.1 Circuit Configuration ............................................. 41

4.2.2 Current Waveforms ............................................41

4.2.3 The Line Current THD and the Input PF ............................43

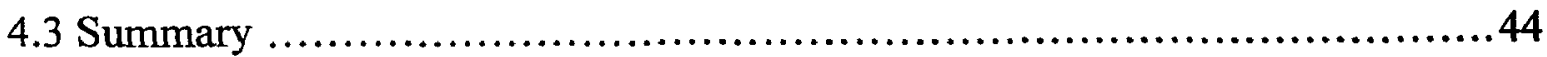


Chapter 5 24-Pulse SCR Rectifier 45

5.1 24-Pulse SCR Rectifier with Inductive Loads ............................. 45

5.1.1 Circuit Configuration .............................................. 45

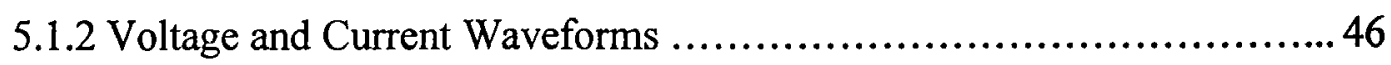

5.1.3 Analysis of Harmonic Elimination ................................. 48

5.1.4 The Line Current THD and the Input PF.............................48

5.2 24-Pulse SCR Rectifier with Capacitive Loads .................................49

5.2.1 Circuit Configuration .............................................49

5.2.2 Current Waveforms ...............................................49

5.2.3 The Line Current THD and the Input PF............................ 51

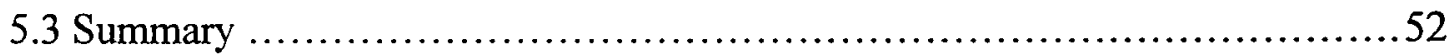

Chapter 6 Experimental Verification of the 12-Pulse SCR Rectifier 53

6.1 Hardware Configuration of the Experimental System .........................53

6.2 Parameter Design of the Experimental System ................................. 56

6.3 Software Programming and Hardware Implementation of the Experimental

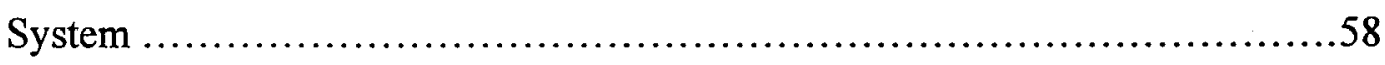

6.3.1 Software Programming ............................................... 58

6.3.2 Hardware Implementation .................................... 58

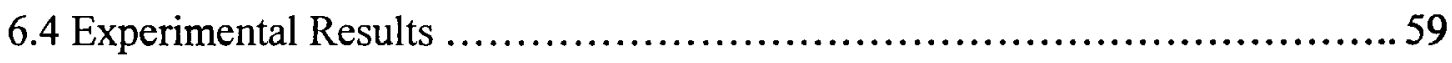

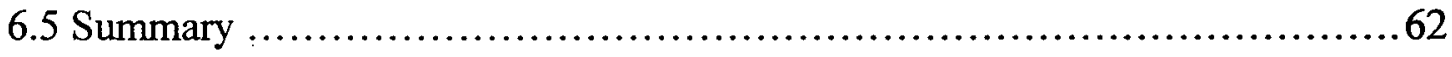

Chapter 7 Conclusions $\quad 65$

Appendix A MC68HC11 Programming $\quad 67$

Appendix B THD and PF Curves of the Six- and Multi-pulse SCR Rectifiers 77

References $\quad 79$ 


\section{List of Figures}

Configuration of the six-pulse SCR rectifier with two devices connected in series.

Simplified diagram of the 12-Pulse SCR rectifier. 4

Circuit configuration of the six-pulse SCR rectifier with an inductive load. 7

Load voltage waveforms of the six-pulse SCR rectifier with inductive loads at $L_{\mathrm{s}}=0$.

2-3 Calculate the average load voltage $V_{L, a v g}$ at $L_{s}=0$.

2-4 Current waveforms of the six-pulse SCR rectifier with inductive loads at $L_{s}=0.11$

2-5 Commutation between $S C R_{l}$ and $S C R_{3}$.

2-6 Voltage and current waveforms with commutation intervals.

2-7 THD and PF curves of the six-pulse SCR rectifier with inductive loads.

2-8 Circuit configuration of the six-pulse SCR rectifier with a capacitive load.

2-9 Current waveforms corresponding to voltage waveforms for the capacitive load condition.

2-10 Waveform and harmonic spectrum of $I_{a l}=1 \mathrm{pu}$ and $I_{a l}=0.2 \mathrm{pu}$ (six-pulse). $\quad 19$

2-11 THD and PF curves of the six-pulse SCR rectifier with capacitive loads. $\quad 20$

3-1 12-pulse SCR rectifier with an inductive load.

3-2 Voltage and current waveforms of the 12-pulse SCR rectifier with inductive loads.

3-3 Calculate the average load voltage of the 12-pulse SCR rectifier with inductive loads.

3-4 Find the phase displacements of harmonics in the delta-connected winding line current when the transformer is $-30^{\circ}$ phase shifted.

3-5 Positive and negative sequence three-phase voltage phasors. 28

3-6 Phase shift of positive sequence and negative sequence currents.

3-7 Phasor relation of the reflected harmonics being cancelled in the primary winding of the 12-pulse SCR rectifier. 
3-8 Compare harmonic spectrum of $i_{a s l}, i_{a s 2}$ and $i_{A}$.

3-9 THD and PF curves of the 12-pulse SCR rectifier with inductive loads. $\quad 32$

3-10 12-pulse SCR rectifier with a capacitive load. 32

3-11 Current waveforms of the 12-pulse SCR rectifier with capacitive loads. 33

3-12 Waveform and harmonic spectrum of $I_{a l}=1 \mathrm{pu}$ and $I_{a l}=0.2 \mathrm{pu}$ (12-pulse). $\quad 34$

3-13 THD and PF curves of the 12-pulse SCR rectifier with capacitive loads. $\quad 35$

4-1 18-pulse SCR rectifier with an inductive load. $\quad 38$

4-2 Waveforms of the 18-pulse SCR rectifier with inductive loads. 39

4-3 Phasor relation of the reflected harmonics being cancelled in the primary winding of the 18-pulse SCR rectifier. $\quad 40$

4-4 THD and PF curves of the 18-pulse SCR rectifier with inductive loads. $\quad 41$

4-5 18-pulse SCR rectifier with a capacitive load. $\quad 41$

4-6 Waveforms of the 18-pulse SCR rectifier with capacitive loads. 42

4-7 Waveform and harmonic spectrum of $I_{a l}=1 \mathrm{pu}$ and $I_{a l}=0.2 \mathrm{pu}$ (18-pulse). $\quad 43$

4-8 THD and PF curves of the 18-pulse SCR rectifier with capacitive loads. $\quad 44$

5-1 24-Pulse SCR rectifier with an inductive load. $\quad 46$

5-2 Waveforms of the 24-pulse SCR rectifier with inductive loads. 47

5-3 Phasor relation of the reflected harmonics being cancelled in the primary winding of the 24-pulse SCR rectifier. 48

5-4 THD and PF curves of the 24-pulse SCR rectifier with inductive loads. 49

5-5 24-pulse SCR rectifier with a capacitive load. 49

5-6 Waveforms of the 24-pulse SCR rectifier with capacitive loads. 50

5-7 Waveform and harmonic spectrum of $I_{a l}=1 \mathrm{pu}$ and $I_{a l}=0.2 \mathrm{pu}$ (24-pulse). $\quad 51$

5-8 THD and PF curves of the 24-pulse SCR rectifier with capacitive loads. 52

6-1 Hardware configuration of the experimental system. 53

6-2 Configuration of the phase-shifting transformer. 55

6-3 Measured source voltage waveforms. 59

6-4 Measured $v_{a c}$ and $v_{Z C}$. $\quad 60$

6-5 Gating pulses generated by the MC68HC11 control board. 60

6-6 Experimental results versus simulation results. 63 


\section{List of Acronyms}

- distortion factor

F - displacement power factor

$\mathrm{O}$ - gate turn-off thyristor

BT - insulated gate bipolar transistor

_. .'L - Kirchhoff's voltage law

$\mathrm{PF}$ - power factor

RMS - root mean square

SCR - silicon controlled rectifier

TDD - total demand distortion

THD - total harmonic distortion 


\section{Chapter 1 Introduction}

Silicon Controlled Rectifier (SCR) is a controllable semiconductor device widely used in high power conversion and control in industry. Modern SCRs are available with high voltage (up to $12 \mathrm{KV}$ ) and current (up to $5 \mathrm{KA}$ ) ratings, which have made the SCR a dominant device in high power converters. High power ac-dc rectifiers, high power battery chargers, load commutated current source inverter powered motor drives and high-voltage direct-current transmission systems are typical applications of SCRs in industry $[3,4]$.

The switching operation of SCRs in a rectifier produces a series of current harmonics, which could be transmitted into the power supply grid. The harmonics in the grid increase wire and transformer losses, cause metering conflicts, and interfere with other equipment connected to the grid [5-7]. Therefore, they have to be restricted within a critical limit.

IEEE Standard 519-1992 defines harmonic limits for electrical power systems. Table 1-1 is extracted from the standard, which is applicable to six-pulse rectifier loads. For multi-pulse rectifier loads, the limits are increased by a factor of $\sqrt{q / 6}$ under certain conditions [1], in which $q$ is the pulse number of the rectifier. However, since only ac voltages are available from the utility supply, rectifier equipment could be considered as dc power generator, converting ac to dc; hence based on Table 1-1, the Total Demand Distortion (TDD) generated by them should be limited within $5 \%$.

Since TDD is expressed as a percentage of the maximum fundamental demand load current (15 or 30 minutes demand) measured after installation, it's not available at the design stage. The Total Harmonic Distortion (THD) based on the percentage of the fundamental rated load current then is calculated in the thesis to provide a reference for evaluating the rectifiers' side effect to the power grid in theory. 
Table 1-1 Harmonic Current Requirement Defined by IEEE Standard 519-1992

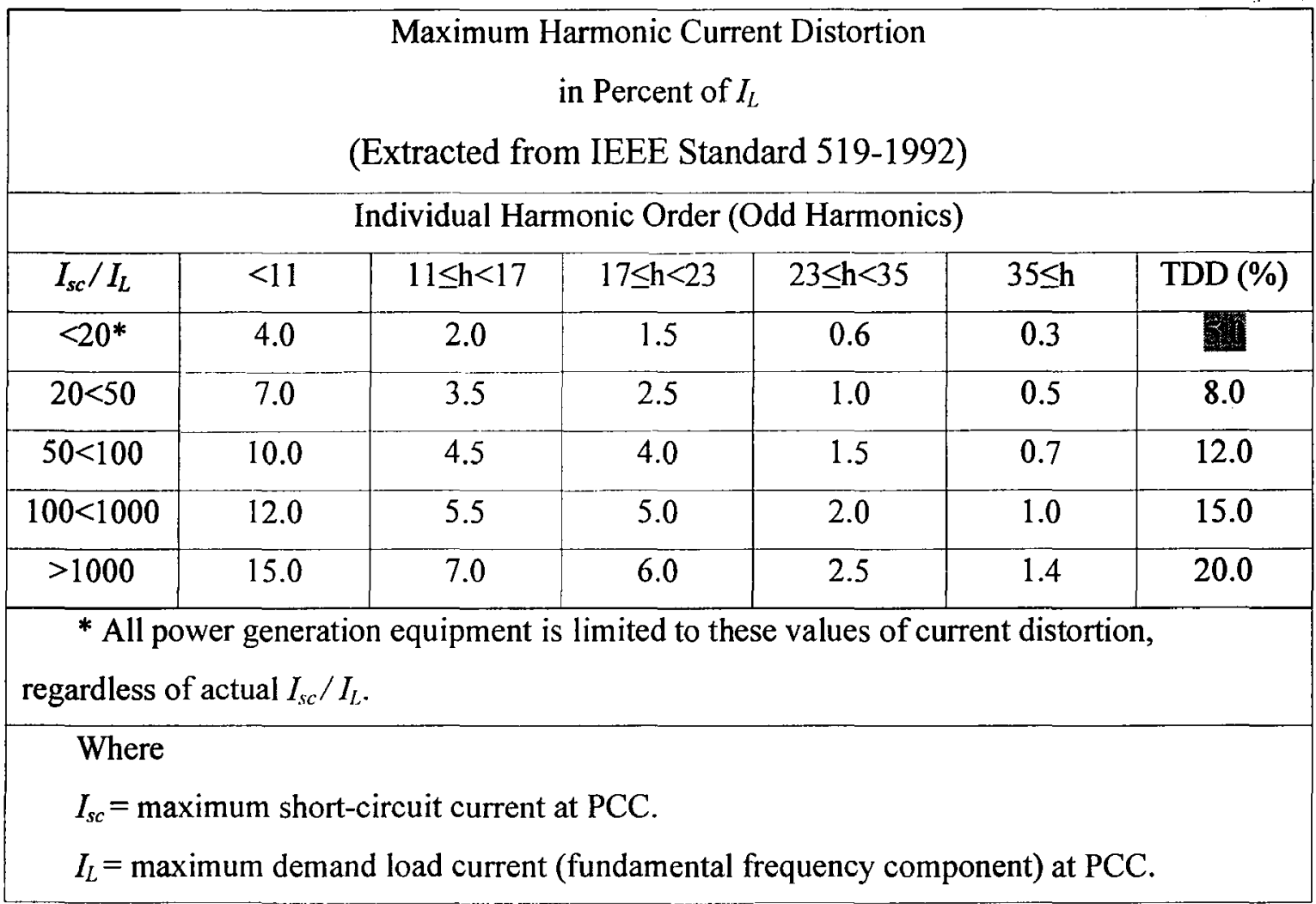

To meet the restriction of IEEE Standard 519-1992, many methods could be applied to eliminate or reduce harmonic currents generated by rectifiers. For example,

(i) Using passive filters. Line reactors (inductors) are often used in conjunction with capacitors in a rectifier to filter specific harmonic currents. However, the connection of capacitors could cause resonance conditions that can magnify harmonic current at certain frequency to a harmful level [6-7].

(ii) Using certain switching techniques. Switching techniques, for instance the pulse width modulation technique, could be used to eliminate harmonic currents. In high power applications, the premise for using these techniques is that the switching devices must be the gate-turn-off type, such as GTOs or IGBTs [8-9].

(iii)Using multi-pulse rectifiers. Multi-pulse rectifiers are designed based on phaseshifting transformers, which enable certain harmonics to be cancelled from the rectifiers' line currents. This method is especially practical for harmonic elimination in high power applications $[1-3,9,10]$. 
This thesis provides full analysis of multi-pulse SCR rectifiers for engineering reference, because they are widely used in industry and play an important role in harmonic eliminations.

This chapter begins with the introduction of the six-pulse SCR rectifier, and then steps into multi-pulse SCR rectifiers, which overcome shortcomings of the six-pulse SCR rectifier. The objective of the thesis is also presented. At the end of the chapter, the outline of the thesis is provided.

\subsection{Introduction of the Six-pulse SCR Rectifier}

It is known that the harmonic currents generated by the six-pulse SCR rectifier in steady state are non triplen odd harmonics - the $5^{\text {th }}, 7^{\text {th }}, 11^{\text {th }}$ and $13^{\text {th }}$, for example. From the spectrum analysis, the dominant low order harmonic currents have higher amplitudes and produce a high $(>20 \%)$ line current THD, which does not meet the requirement of IEEE Standard 519-1992. Therefore they have to be eliminated or decreased.

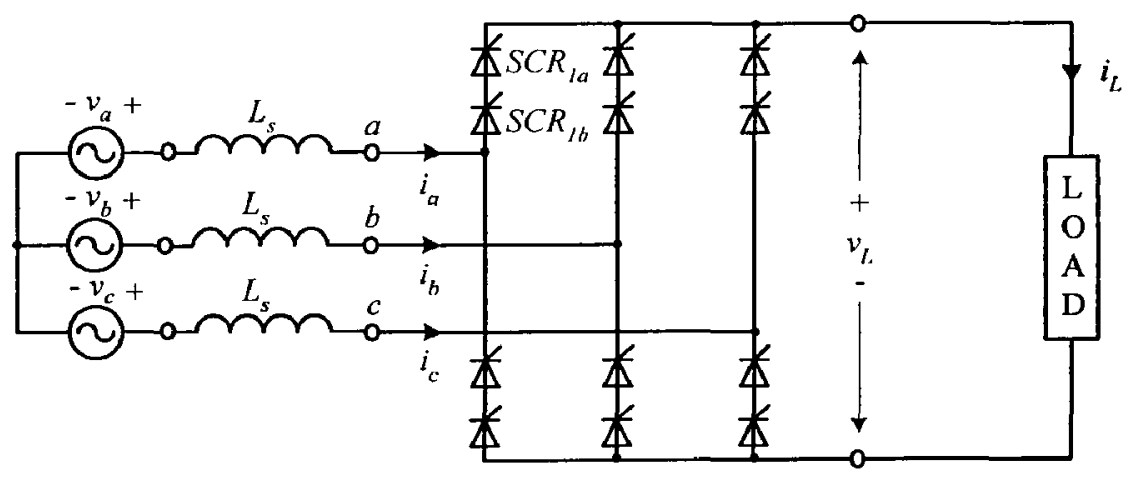

Fig. 1-1 Configuration of the six-pulse SCR rectifier with two devices connected in series.

In the six-pulse SCR rectifier, it might be required to connect SCRs in series in large horsepower applications to block high voltages, under which a single SCR cannot handle. Voltage sharing is the main problem of the component in-series connection. Fig. 1-1 shows the configuration of the six-pulse $S C R$ rectifier with two devices connected in series. The two SCRs, for instance $S C R_{l a}$ and $S C R_{I b}$, should be turned on (or turned off) simultaneously. If not, the one turned on later (or turned off earlier) will undertake a 
higher voltage and thus may be damaged. If more SCRs are required to be in series in high-voltage applications, the voltage-sharing problem becomes more critical, because the synchronized switching is more difficult to realize.

\subsection{Introduction of Multi-pulse SCR Rectifiers}

To eliminate harmonics from the line current of the six-pulse SCR rectifier, and to avoid in-series SCRs in high power applications, multi-pulse SCR rectifiers using phaseshifting transformers are developed. The 12-, 18- and 24-pulse SCR rectifiers are commonly applied in industry.

Fig. 1-2 shows the simplified diagram of the 12-pulse SCR rectifier, which consists of a phase-shifting transformer and two six-pulse SCR bridges that are connected in series on the dc side. Fig. 1-2(a) shows the inductive load condition, for example load commutated current source inverter powered motor drives or high-voltage direct-current transmission systems. Fig. 1-2(b) shows the capacitive load condition, such as high power battery chargers or de motor drives.

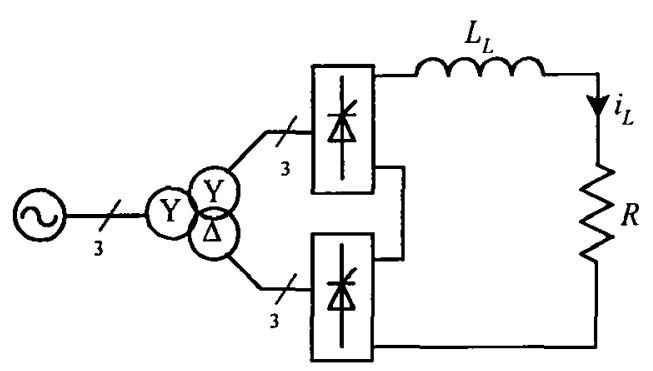

(a) with inductive loads

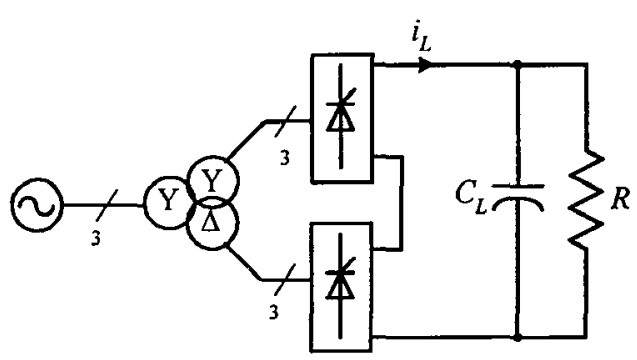

(b) with capacitive loads

Fig. 1-2 Simplified diagram of the 12-Pulse SCR rectifier.

The main feature of the 12-pulse SCR rectifier is that two SCR bridges are connected in series rather than two SCRs, thus the voltage sharing is avoided. As a result, the system becomes more reliable. Another advantage of the 12-pulse SCR rectifier is that the line 
current THD is reduced substantially owing to the use of the phase-shifting transformer. The details of the harmonic elimination will be analyzed in Chapter 3.

For the 18-pulse SCR rectifier, a phase-shifting transformer with three secondary windings is connected to three six-pulse SCR bridges. Similarly, connecting a phaseshifting transformer with four secondary windings to four six-pulse bridges introduces the 24-pulse rectifier. Since more SCR bridges are connected in series to tolerate high voltages, and more low-order harmonics are eliminated from the line current, the 18-and 24-pulse SCR rectifiers are widely used in high power applications. For example, they could be applied as front ends of high power motor drive systems.

\subsection{Thesis Objectives}

As multi-pulse SCR rectifiers overcome shortcomings of the six-pulse SCR rectifier and play an important role in high power applications, the main objective of the thesis is to analyze the performance of multi-pulse SCR rectifiers. The study is focused on the line current THD, the input Power Factor (PF), and the harmonic elimination theory. To achieve this objective, main tasks are outlined as follows:

- To use computer simulation to investigate the performance of the six-pulse SCR rectifier and multi-pulse SCR rectifiers with inductive and capacitive loads.

- To compare the performance of the six-pulse rectifier and multi-pulse rectifiers by providing voltage and current waveforms under different load conditions.

- To derive formulas for evaluating rectifiers' performance, such as the average load voltage and current, the RMS and THD of the line current, as well as the input PF of the rectifier.

- To analyze the harmonic elimination theory by Fourier analysis and positive/negative sequence analysis, respectively.

- To obtain the line current THD and the input PF under different operating conditions by simulations.

- To verify the performance of the 12-pulse SCR rectifier experimentally. 


\subsection{Thesis Outline}

The outline of the following chapters is as follows:

Chapter 2 presents the performance of the six-pulse SCR rectifier with inductive and capacitive loads, respectively. The load voltage and current, the RMS and THD of the line current and the input PF of the rectifier under ideal and non-ideal conditions are discussed.

Chapter 3 presents the performance of the 12-pulse SCR rectifier. The harmonic elimination theory is illustrated by Fourier analysis and positive/negative sequence analysis, respectively. Voltage and current waveforms, harmonic spectrum and THD of the line current as well as the input PF under different operating conditions are provided.

Chapter 4 and 5 presents the performance of the 18- and 24-pulse SCR rectifiers, respectively. Typical voltage and current waveforms, harmonic spectrum and THD of the line current, the harmonic elimination theory and the input PF are discussed.

Chapter 6 presents the experimental verification of the 12-pulse SCR rectifier. Typical voltage and current waveforms are obtained and compared with simulation results.

Chapter 7 provides conclusions of the thesis. 


\section{Chapter 2 Six-pulse SCR Rectifier}

The six-pulse SCR rectifier is a fundamental three-phase controllable ac-dc converter composed of six SCR devices. It is also the building block of multi-pulse SCR rectifiers. For example, a 12-pulse SCR rectifier consists of two six-pulse bridges, and the 18- and 24-pulse rectifiers include three and four six-pulse bridges, respectively. The line current Total Harmonic Distortion (THD) of various rectifiers is of great interest owing to the need of complying with the harmonic current requirement defined by IEEE Standard 5191992 and evaluating the rectifiers' side effect to the power supply grid. The efficiency of the rectifier can be scaled by the parameter of the input Power Factor (PF).

This chapter presents the performance of the six-pulse SCR rectifier with inductive and capacitive loads. Voltage and current waveforms are provided to show the operation of the rectifier. For the inductive load condition, the average load voltage and current under ideal and non-ideal circuit conditions are derived; the RMS and THD of the line current are analyzed; the input PF is calculated. For the capacitive load condition, the line current THD and the input PF are discussed.

\subsection{Six-Pulse SCR Rectifier with Inductive Loads}

\subsubsection{Circuit Configuration}

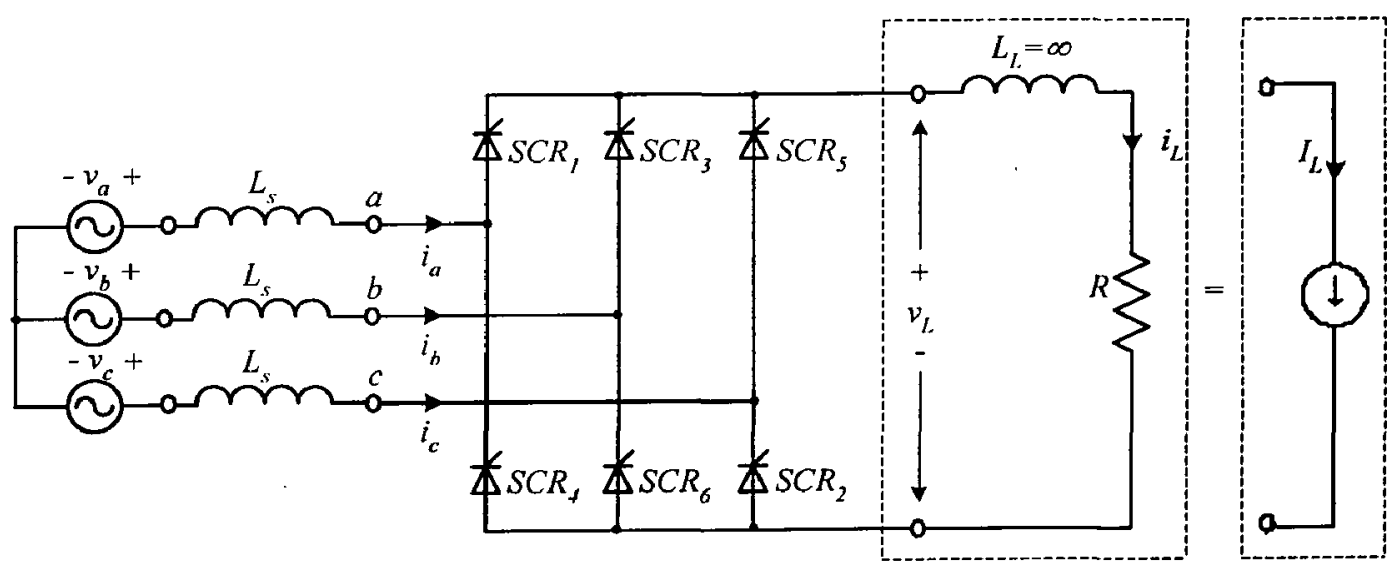

Fig. 2-1 Circuit configuration of the six-pulse SCR rectifier with an inductive load. 
Fig. 2-1 shows the circuit configuration of the six-pulse SCR rectifier with an inductive load. The input of the SCR rectifier is a balanced three-phase power supply, which consists of the following phase voltages:

$$
\begin{gathered}
v_{a}=\sqrt{2} V_{P H} \sin (\omega t), \\
v_{b}=\sqrt{2} V_{P H} \sin \left(\omega t-\frac{2 \pi}{3}\right), \\
v_{c}=\sqrt{2} V_{P H} \sin \left(\omega t-\frac{4 \pi}{3}\right) .
\end{gathered}
$$

The corresponding line-to-line voltages are:

$$
\begin{gathered}
v_{a b}=v_{a}-v_{b}=\sqrt{2} V_{L L} \sin \left(\omega t+\frac{\pi}{6}\right), \\
v_{b c}=\sqrt{2} V_{L L} \sin \left(\omega t-\frac{\pi}{2}\right), \\
v_{c a}=\sqrt{2} V_{L L} \sin \left(\omega t-\frac{7 \pi}{6}\right),
\end{gathered}
$$

where $V_{P H}$ is the RMS value of the phase voltage, $V_{L L}$ is the RMS value of the line-to-line voltage, and $\omega$ is the angular frequency of the supply voltage.

$L_{s}$ represents the total line inductance between the power supply grid and the rectifier bridge, which includes the equivalent inductance of the power supply and the inductance of a three-phase reactor that is often added to the system to reduce the line current THD.

$L_{L}$ is the dc choke, which is used to reduce the ripple component of the load current. For easy of analysis, assume that the value of $L_{L}$ is infinite, and then the inductive load can be replaced by a dc current source $I_{L}$ as shown in Fig. 2-1.

\subsubsection{Circuit Operation with $L_{s}=0$}

The discussion of the six-pulse SCR rectifier begins with the ideal circuit condition, under which the line inductance $L_{s}$ is zero. 


\section{1) Load Voltage and Current}

Since the gating pulses control the conduction of the SCRs, the output voltage of the six-pulse SCR rectifier relates to the so-called firing angle or delay angle $\alpha$. Fig. 2-2 shows the load voltage waveforms at different $\alpha$ s. Obviously, the bigger the $\alpha$, the lower the line-to-line voltage will be. Note that the range of the delay angle $\alpha$ is from 0 to $\pi$ radians.
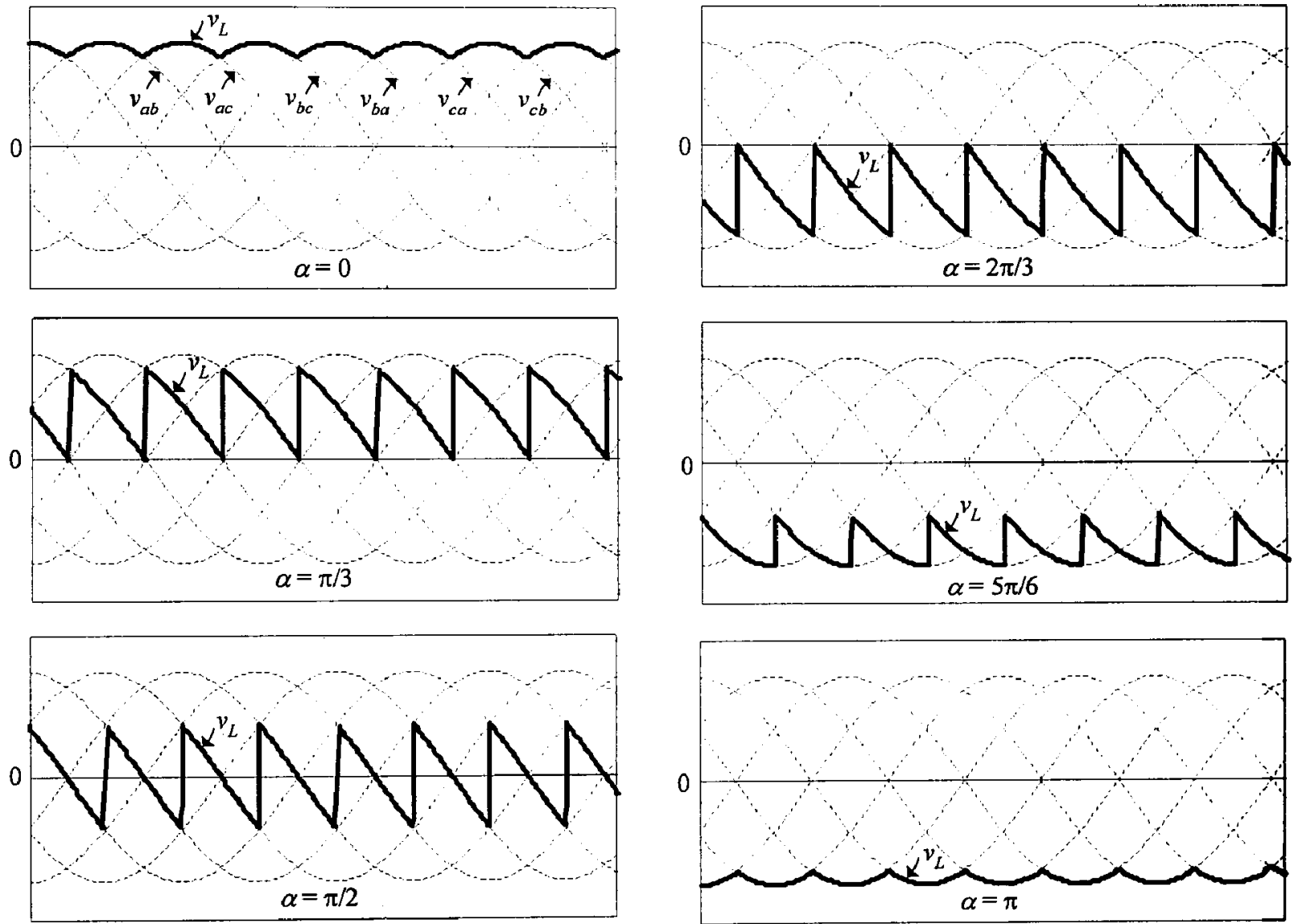

Fig. 2-2 Load voltage waveforms of the six-pulse SCR rectifier with inductive loads at $L_{s}=0$.

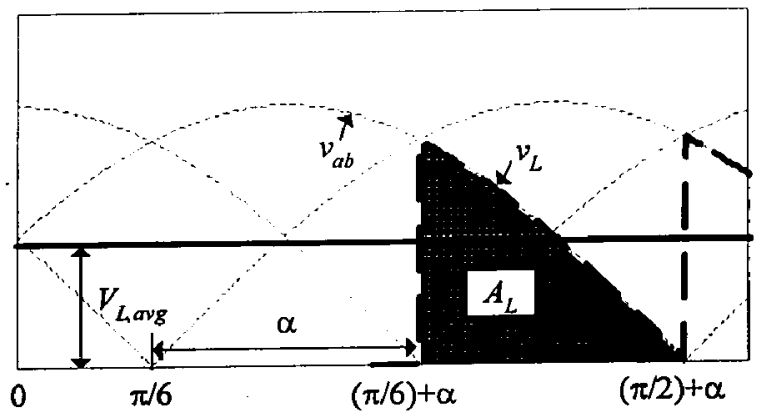

Fig. 2-3 Calculate the average load voltage $V_{L, \text { avg }}$ at $L_{s}=0$. 
From Fig. 2-2, it can be seen that the dc voltage obtained from the six-pulse SCR rectifier contains six ripples in one supply voltage cycle. Based on Fig. 2-3, we could have

$$
\begin{aligned}
& V_{L, a v g}=\frac{\text { area } A_{L}}{\pi / 3}=\frac{1}{\pi / 3} \int_{\pi / 6+\alpha}^{\pi / 2+\alpha} v_{a b} d(\omega t)=\frac{1}{\pi / 3} \int_{\pi / 6+\alpha}^{\pi / 2+\alpha} \sqrt{2} V_{L L} \sin \left(\omega t+\frac{\pi}{6}\right) d(\omega t), \\
& =\frac{3 \sqrt{2}}{\pi} V_{L L} \cos \alpha=1.35 V_{L L} \cos \alpha
\end{aligned}
$$

where $V_{L, a v g}$ is the average load voltage.

From equation (2.7), when the delay angle $\alpha$ is less than $90^{\circ}$, the average load voltage is positive. The energy is transferred from the source to the load. In this condition, the $\mathrm{SCR}$ rectifier is called operating in the rectification mode. When the delay angle $\alpha$ is greater than $90^{\circ}$, the average load voltage becomes negative. The energy is transferred from the load to the source. The rectifier is called working in the inverting mode.

Fig. 2-4 shows the current waveforms of the six-pulse SCR rectifier. $i_{S C R l}, i_{S C R 3}$ and $i_{S C R S}$ are the currents passing through $S C R_{1}, S C R_{3}$ and $S C R_{5}$, each of which conducts for $2 \pi / 3$ radians in one supply voltage cycle in turn. The similar condition happens to $S C R_{2}, S C R_{4}$ and $S C R_{6}$ as well, because at any moment there must be two SCRs conducting, one $\left(S C R_{l}, S C R_{3}\right.$ or $\left.S C R_{5}\right)$ connected to the positive pole of the rectifier bridge, and the other $\left(S C R_{2}, S C R_{4}\right.$ or $\left.S C R_{6}\right)$ to the negative pole, to let the current flow. The waveform of $i_{S C R 2}$ and $i_{S C R 6}$ are not shown in Fig. 2-4, but they can be easily obtained by referencing to the $i_{S C R 4}$ waveform. The line current of phase A can be calculated by $i_{a}=$ $i_{S C R I}-i_{S C R 4}$. Thus $i_{a}$ consists of a positive part and a negative part, which are contributed by $S C R_{l}$ and $S C R_{4}$, respectively. $i_{L}$ is the load current, which is the sum of $i_{S C R l}, i_{S C R 3}$ and $i_{S C R} . I_{L}$ stands for the amplitude of $i_{L}$.

Since the average load current $I_{L, a v g}$ can be obtained by

$$
I_{L, \text { avg }}=\frac{V_{L, \text { avg }}}{R},
$$

$I_{L}$ equals to $I_{L, a v g}$. 

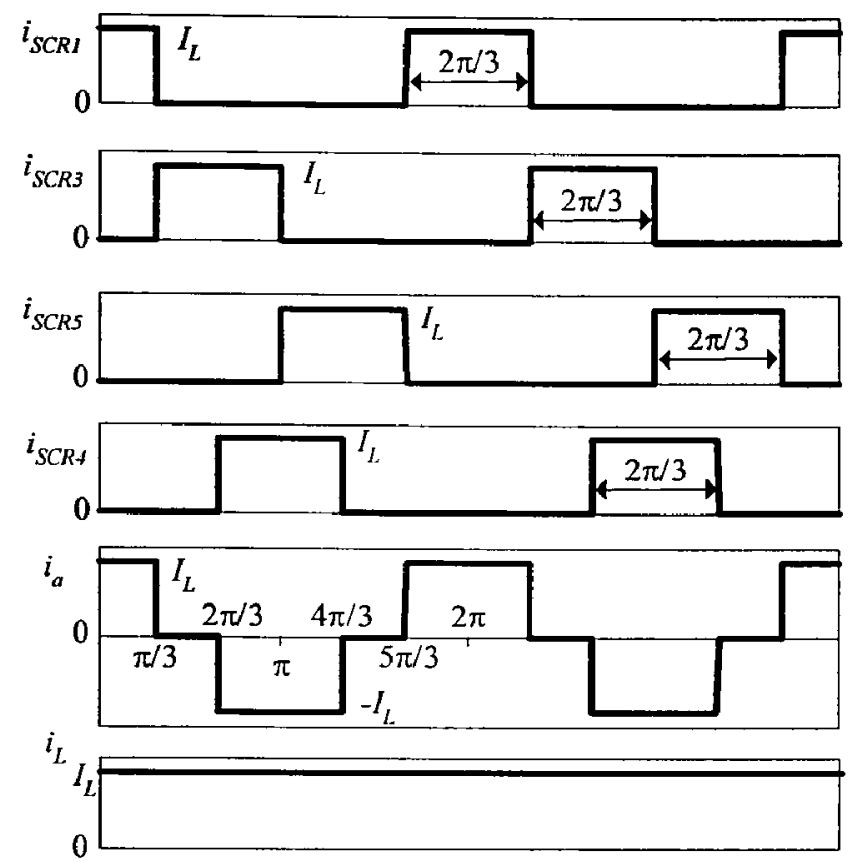

Fig. 2-4 Current waveforms of the six-pulse SCR rectifier with inductive loads at $L_{s}=0$.

The RMS value of the dc load current equals to its average value, i.e.:

$$
I_{L, R M S}=I_{L, \text { avg }} \text {. }
$$

The load power is defined as:

$$
P_{L}=I_{L, R M S}^{2} \times R
$$

Since SCRs in each phase conduct one-third of one supply voltage cycle ( $2 \pi / 3$ radians), we have

$$
I_{S C R, a v g}=\frac{I_{L, a v g}}{3}
$$

where $I_{S C R \text {,avg }}$ is the average current in each SCR. 


\section{2) Line Current}

\section{RMS Value of the Line Current}

Based on Fig. 2-4, the RMS value of the line current can be calculated by

$$
\begin{aligned}
& I_{a, R M S}=\sqrt{\frac{1}{2 \pi} b^{2 \pi}\left(i_{a}\right)^{2} d(\omega t)} \\
& =\sqrt{\frac{1}{2 \pi}\left[b^{\pi / 3}\left(I_{L}\right)^{2} d(\omega t)+\int_{2 \pi / 3}^{4 \pi / 3}\left(-I_{L}\right)^{2} d(\omega t)+f_{5 \pi / 3}^{2 \pi}\left(I_{L}\right)^{2} d(\omega t)\right] .} \\
& =\sqrt{\frac{2}{3}} I_{L}=0.816 I_{L}
\end{aligned}
$$

Hence once the amplitude of the dc load current is known, the RMS value of the line current can be calculated.

\section{THD of the Line Current}

The line current of the six-pulse SCR rectifier is no longer a sine wave because of the harmonics. From Fourier analysis, the harmonic content of $i_{a}$ can be calculated.

In Fig. $2-4, i_{a}$ is shown as an even function with period $T=2 \pi$. Thus it can be expressed by the following Fourier Series:

$$
i_{a}(\omega t)=\frac{a_{0}}{2}+\sum_{n=1}^{\infty} a_{n} \cos \frac{n \pi}{T / 2} \omega t=\frac{a_{0}}{2}+\sum_{n=1}^{\infty} a_{n} \cos n \omega t,
$$

where $a_{0}$ is the dc component in $i_{a}$, which is zero, and $a_{n}$ is defined as

$$
\begin{aligned}
& a_{n}=\frac{2}{T / 2} b^{T / 2} i_{a}(\omega t) \cos \frac{n \pi}{T / 2} \omega t d(\omega t)=\frac{2}{\pi} b_{b}^{\pi} i_{a}(\omega t) \cos n \omega t d(\omega t) \\
& =\frac{2}{\pi}\left[b^{\pi / 3} I_{L} \cos n \omega t d(\omega t)+\mathfrak{l}_{2 \pi / 3}^{\pi}\left(-I_{L}\right) \cos n \omega t d(\omega t)\right] \\
& =\frac{2 I_{L}}{n \pi}\left[\sin \left(\frac{n \pi}{3}\right)+\sin \left(\frac{2 n \pi}{3}\right)\right] \quad(n=0,1,2, \cdots)
\end{aligned} .
$$

Calculate the coefficient $a_{n}$, and consider $\phi_{l}$, the phase angle of $i_{a l}$ with respect to $v_{a}$, we have:

$$
\begin{gathered}
i_{a}=1.1 I_{L}\left[\cos \left(\omega t-\phi_{1}\right)-\frac{1}{5} \cos 5\left(\omega t-\phi_{1}\right)+\frac{1}{7} \cos 7\left(\omega t-\phi_{1}\right)\right. \\
\left.-\frac{1}{11} \cos 1\left(\omega t-\phi_{1}\right)+\frac{1}{13} \cos 13\left(\omega t-\phi_{1}\right)-\cdots\right]
\end{gathered} .
$$


It can be noticed that there is no triplen harmonics $\left(3^{\text {rd }}, 6^{\text {th }}, 9^{\text {th }}, 12^{\text {th }} \ldots\right)$ in $i_{a}$, because the rectifier is a balanced three-phase system.

Rewrite equation (2.15) as:

$$
i_{a}=\sum_{n=1,5,7,11}^{\infty} \sqrt{2} I_{a n} \cos n\left(\omega t-\phi_{1}\right)
$$

where $I_{a n}$ is the RMS value of each harmonic component in $i_{a}$, then the RMS value of $i_{a}$ can be expressed as:

$$
I_{a, R M S}=\sqrt{\sum_{n=1,5,7,11}^{\infty} I_{a n}^{2}}
$$

The THD of $i_{a}$ is defined by:

$$
T H D_{i a}=\frac{\sqrt{I_{a, R M S}^{2}-I_{a 1, R M S}^{2}}}{I_{a 1, R M S}},
$$

where $I_{a l, R M S}$ is the RMS value of $i_{a l}$, the fundamental component of $i_{a}$. From equation (2.15), the amplitude of $i_{a I}$ can be obtained by:

$$
I_{a 1, m}=1.1 I_{L}
$$

then

$$
I_{a 1, R M S}=\frac{I_{a 1, m}}{\sqrt{2}}=0.78 I_{L} .
$$

Substitute equations (2.12) and (2.20) to (2.18), we have:

$$
T H D_{i a}=\frac{\sqrt{I_{a, R M S}^{2}-I_{a 1, R M S}^{2}}}{I_{a 1, R M S}}=\frac{\sqrt{\left(0.816 I_{L}\right)^{2}-\left(0.78 I_{L}\right)^{2}}}{0.78 I_{L}}=0.311 .
$$

\section{3) Input PF of the SCR Rectifier}

In a balanced three-phase system, the power factor of the system is equivalent to that of each phase. Per-phase average (real) power can be obtained from the phase voltage and the fundamental component of the phase current, i.e.:

$$
P=\frac{1}{2 \pi} \int_{0}^{2 \pi} v_{a} i_{a} d(\omega t)=V_{a} I_{a 1} \cos \phi_{1} .
$$

Per-phase apparent power is obtained from the phase voltage and the total phase current, i.e.: 


$$
S=V_{a} I_{a}
$$

Then the power factor is:

$$
P F=\frac{P}{S}=\frac{V_{a} I_{a 1} \cos \phi_{1}}{V_{a} I_{a}}=\frac{I_{a 1}}{I_{a}} \cos \phi_{1}=D F \times D P F .
$$

where $D F$ is the distortion factor that is defined as: $D F=I_{a 1} / I_{a}$, and $D P F$ is the displacement power factor that is defined as: $D P F=\cos \phi_{1}$. In the SCR rectifier circuit, $\phi_{1}$ is the result of the delay angle $\alpha$. Under ideal circuit condition, $\phi_{1}=\alpha$; hence $\cos \phi_{1}=\cos \alpha$. From $T H D=\sqrt{I_{a}^{2}-I_{a 1}^{2}} / I_{a 1}$, we have $I_{a 1} / I_{a}=1 / \sqrt{\left(1+T H D^{2}\right)} ;$ therefore

$$
P F=\frac{D P F}{\sqrt{1+T H D^{2}}}=\frac{\cos \alpha}{\sqrt{1+T H D^{2}}}=0.955 \cos \alpha \text {. }
$$

\subsubsection{Circuit Operation with $L_{s} \neq 0$}

\section{1) Commutation Interval and Corrected Load Voltage and Current}

In Fig. 2-1, when the line inductance $L_{s}$ is not zero, the SCRs in the rectifier will no longer turn on or turn off instantaneously. Instead, a commutation interval appears. Fig. 2-5 shows a part of the SCR rectifier circuit, in which commutation occurs between $S C R_{I}$ and $S C R_{3}$. Fig. 2-6 shows the voltage and current waveforms with $L_{s} \neq 0$. In Fig. 2-6(a), due to the $L_{s}$, the current in $S C R_{I}$ reduces from $I_{L}$ to zero gently and the current in $S C R_{3}$ increases from zero to $I_{L}$ gradually. Therefore, there exists a short commutation interval $\gamma$.

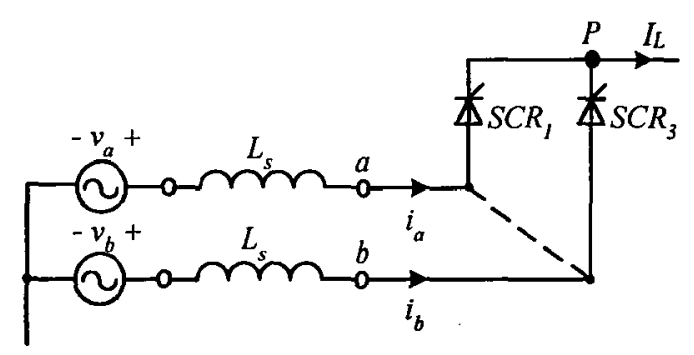

Fig. 2-5 Commutation between $S C R_{1}$ and $S C R_{3}$. 


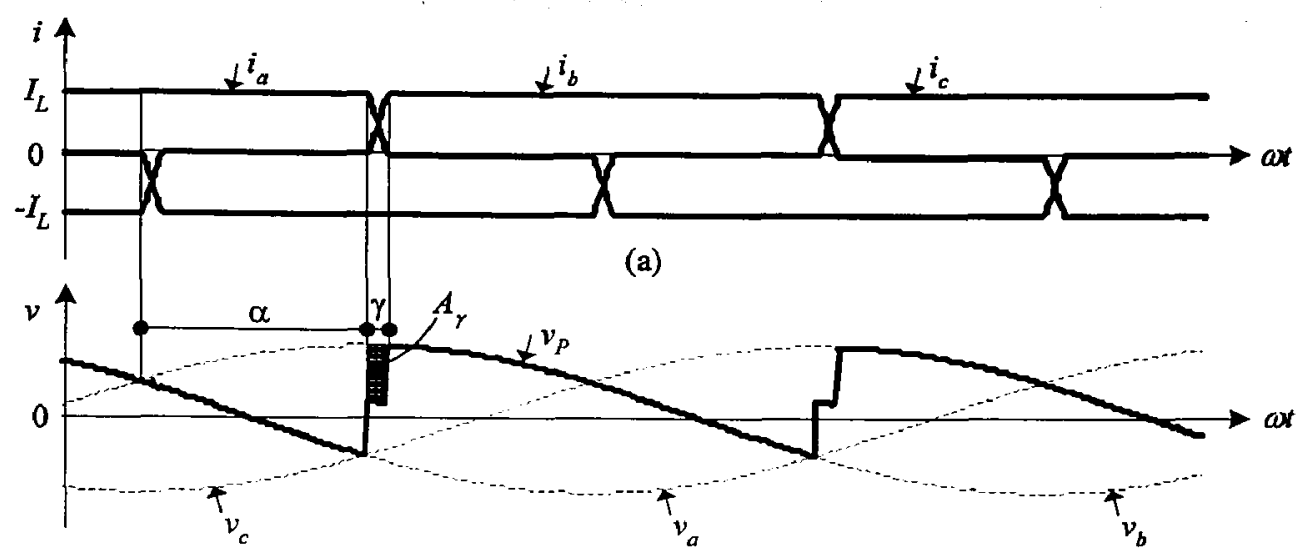

(b)

Fig. 2-6 Voltage and current waveforms with commutation intervals.

During the commutation interval $\gamma$, as $S C R_{1}$ and $S C R_{3}$ are both on, the source is shortcircuited (indicated by the dotted line in Fig. 2-5). Therefore, there would be an output voltage loss, which is indicated by the shaded area in Fig. 2-6(b). Based on the Kirchhoff's Voltage Law (KVL) at node $P$ in Fig. 2-5, we have

$$
v_{P}=v_{a}-L_{s} \frac{d i_{a}}{d t}=v_{b}-L_{s} \frac{d i_{b}}{d t}
$$

From which:

$$
v_{P}=\frac{v_{a}+v_{b}}{2}-\frac{L_{s}}{2}\left(\frac{d i_{a}}{d t}+\frac{d i_{b}}{d t}\right)
$$

Since $\frac{d i_{a}}{d t}=-\frac{d i_{b}}{d t}$, then

$$
\frac{d i_{a}}{d t}+\frac{d i_{b}}{d t}=\mathbf{0}
$$

Hence

$$
v_{P}=\frac{v_{a}+v_{b}}{2} .
$$

The shaded area $A_{y}$ in Fig. 2-6(b) can be expressed as:

$$
A_{\gamma}=\int_{\alpha}^{\alpha+\gamma}\left(v_{b}-v_{P}\right) d(\omega t) .
$$

Since $v_{b}-v_{P}=L_{s} \frac{d i_{b}}{d t}$, we can write 


$$
A_{\gamma}=\int_{\alpha}^{\alpha+\gamma} L_{s} \frac{d i_{b}}{d t} d(\omega t)=\int_{0}^{I_{L}} \omega L_{s} d i_{b}=\omega L_{s} I_{L}
$$

Then the average dc voltage loss is:

$$
\Delta V=\frac{A_{\gamma}}{\pi / 3}=\frac{3 \omega L_{s} I_{L}}{\pi} .
$$

From equation (2.32), we know that the average dc voltage loss is proportional to $L_{s}$ and $I_{L}$. The corrected average load voltage $V_{L_{1} a v g_{-} c}$ and current $I_{L, a v g_{-} c}$ are:

$$
\begin{gathered}
V_{L, a v g_{-} c}=V_{L, a v g}-\Delta V, \\
I_{L, a v g_{-} c}=\frac{V_{L, a v g_{-} c} .}{R} .
\end{gathered}
$$

The commutation interval can be calculated by

$$
\begin{aligned}
& A_{\gamma}=\int_{\alpha}^{\alpha+\gamma}\left(v_{a}-v_{P}\right) d(\omega t)=\int_{\alpha}^{\alpha+\gamma} v_{a}-\frac{v_{a}+v_{b}}{2} d(\omega t)=\int_{\alpha}^{\alpha+\gamma} \frac{v_{a b}}{2} d(\omega t) \\
& =\frac{V_{L L}}{\sqrt{2}}[\cos \alpha-\cos (\alpha+\gamma)]=\omega L_{s} I_{L}
\end{aligned},
$$

where

$$
\gamma=\cos ^{-1}\left(\cos \alpha-\frac{\sqrt{2} \omega L_{s}}{V_{L L}} I_{L}\right)-\alpha .
$$

\section{2) The Line Current THD and the Input PF}

From equation (2.12), we know that the line current is proportional to the load current. Therefore, the smaller the value of $R$ (heavy load condition), the bigger the line current will be, and the more energy will be stored and released by $L_{s}$. Consequently, a longer commutation interval occurs. On the other hand, a larger $L_{s}$ will extend the commutation interval as well. A longer commutation interval makes the amplitude of $i_{a l}$ higher. As a result, the line current THD becomes lower. Fig. 2-7(a) shows the line current THD curves at different $L_{s}$ values, in which the THD decreases as the $L_{s}$ increases. Fig. 2-7(b) shows the THD curves at different $\alpha$ s. It can be seen that with the same $L_{s}$, the THD increases when $\alpha$ increases.

Fig. 2-7(c) shows the PF curves. From $P F=D P F / \sqrt{1+T H D^{2}}$, we know that the PF is related to THD and DPF. At any fixed $\alpha$, when the load current $I_{L}$ decreases, the RMS 
value of $i_{a}$ decreases. As a result, the phase displacement $\phi_{I}$ decreases, the DPF $\left(=\cos \phi_{1}\right)$ increases, and the PF increases.

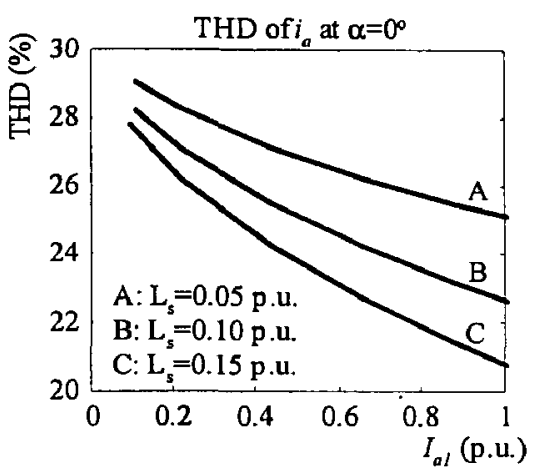

(a)

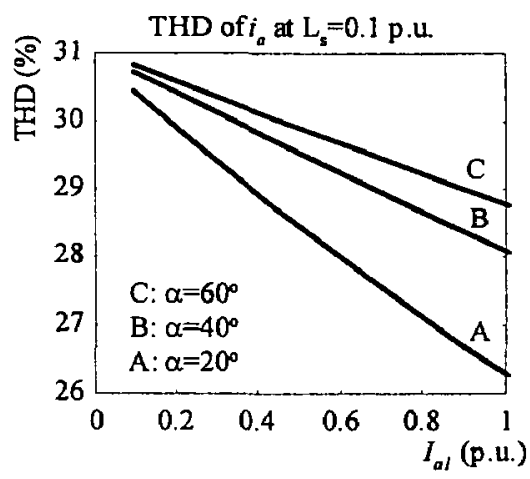

(b)

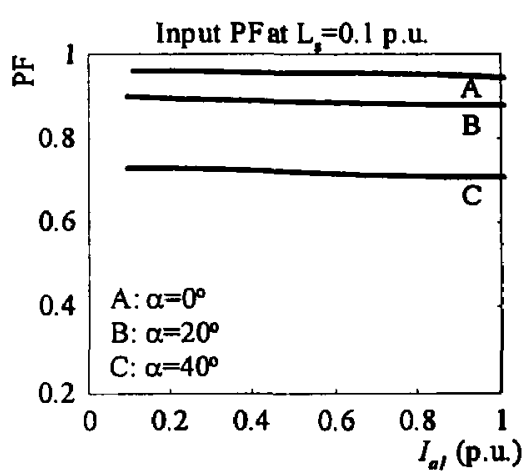

(c)

Fig. 2-7 THD and PF curves of the six-pulse SCR rectifier with inductive loads.

\subsection{Six-Pulse SCR Rectifier with Capacitive Loads}

\subsubsection{Circuit Configuration}

Fig. 2-8 shows the circuit configuration of the six-pulse SCR rectifier with a capacitive load. The capacitor $C$ is used to stabilize the voltage across the load. When the value of $C$ reaches infinite, the capacitive load can be replaced by a dc voltage source with amplitude E. $L_{s}$ is the line inductance, which avoids short circuit between the power supply and the dc voltage source. In practice, $E$ could be the voltage on a capacitive filter, the back emf of a dc motor, or the rated voltage of a rechargeable battery.

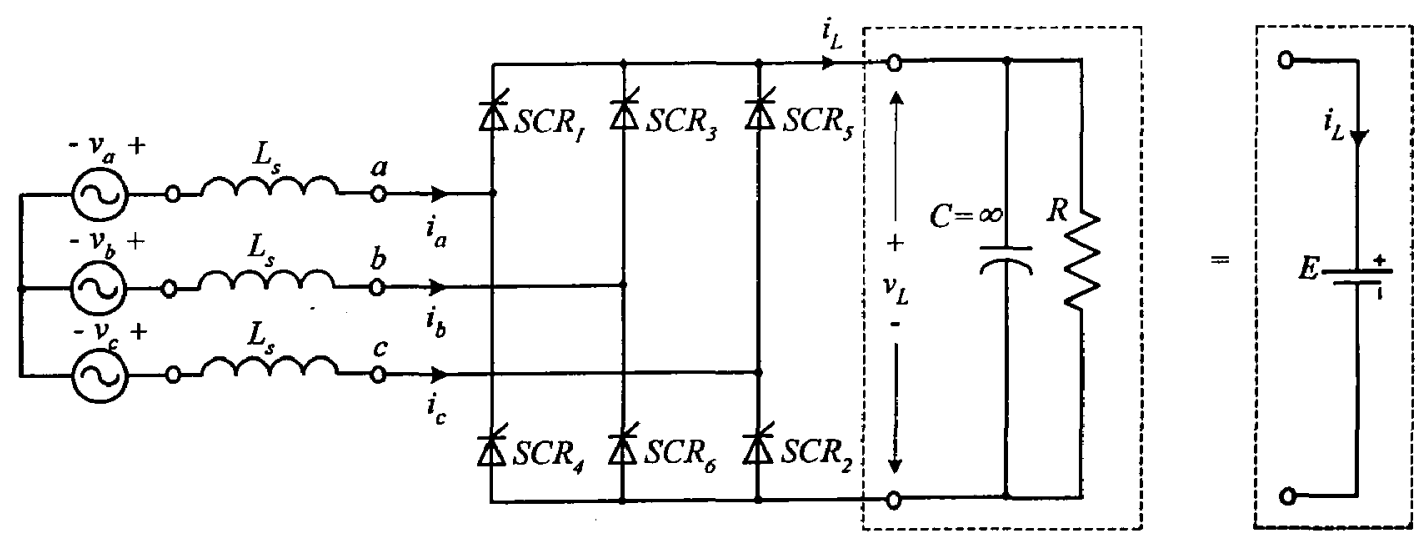

Fig. 2-8 Circuit configuration of the six-pulse SCR rectifier with a capacitive load. 


\subsubsection{Current Waveforms}

Fig. 2-9 shows the current waveforms corresponding to voltage waveforms. When the line-to-line supply voltage $v_{L L}$ is greater than $E$ and the related SCRs are triggered, the line current $i_{a}$ increases gradually and reaches to a peak, at which $v_{L L}=E$; then it begins to decrease till reaches zero. As a result, two humps appear in the positive half cycle of $i_{a}$. The same phenomenon happens to the negative half cycle of $i_{a}$ as well. The load current $i_{L}$ is the summation of the positive half cycle of the three line currents.

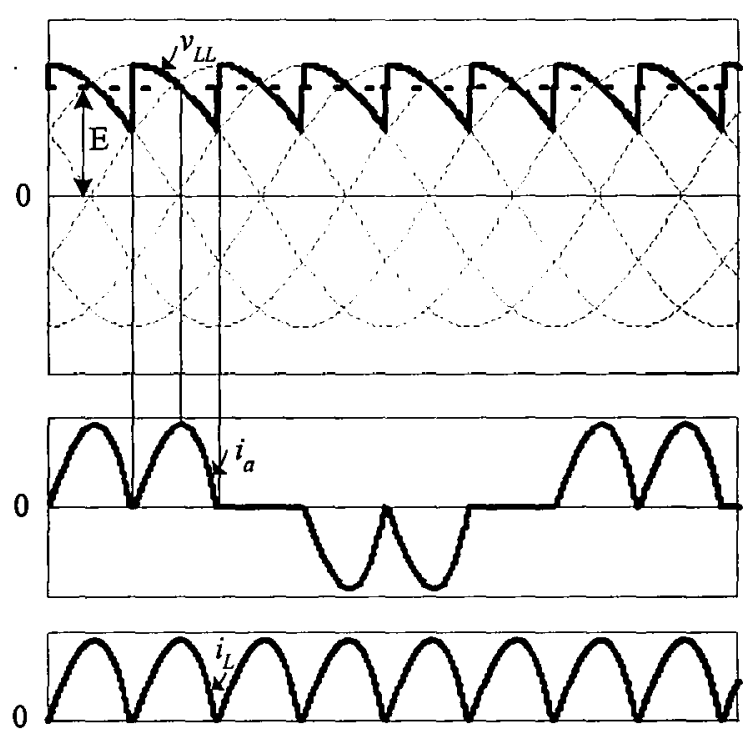

Fig. 2-9 Current waveforms corresponding to voltage waveforms for the capacitive load condition.

The value of $E$ varies slightly with the loading conditions. Under light load conditions, $E$ is close to the peak of $v_{L L}$; therefore the load current $i_{L}$ could be discontinuous. As the.load becomes heavier, a bigger current will be drawn, which causes a larger voltage drop across $L_{s}$; hence $E$ decreases. As a result, the current waveform becomes smoother. When the load increases to a certain level, the load current becomes continuous that indicates a continuous current operation mode of the SCR rectifier. 


\subsubsection{The Line Current THD and the Input PF}

As $L_{s}$ increases, the rising and falling rate of each hump in $i_{a}$ decreases, and the current waveform becomes slightly smoother. In addition, the amplitude of the load current impacts the shape of $i_{a}$. A higher amplitude results in a quite smoother current waveform. Fig. 2-10 shows the waveform and the harmonic spectrum of $I_{a l}=0.2 \mathrm{pu}$ and $I_{a l}=1.0 \mathrm{pu}$, respectively, where $I_{a l}$ is the RMS value of the fundamental component of $i_{a}$. It can be seen that as $I_{a l}$ increases, the shape of $i_{a}$ turns smoother, and the THD of $i_{a}$ becomes lower.
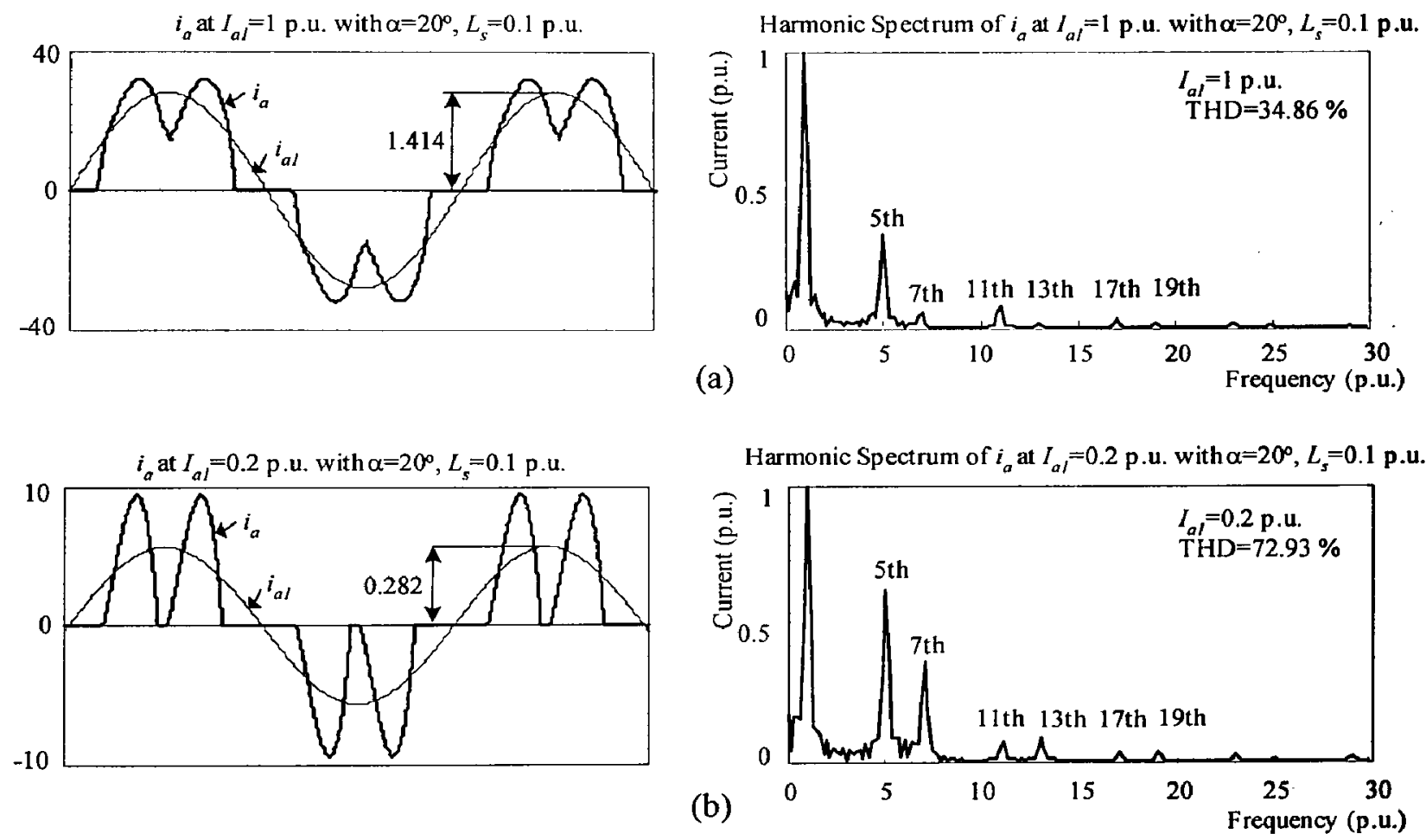

Fig. 2-10 Waveform and harmonic spectrum of $I_{a l}=1 \mathrm{pu}$ and $I_{a f}=0.2 \mathrm{pu}$.

Fig. 2-11(a) and (b) show the THD curves at different value of $L_{s}, I_{a l}$ and $\alpha$. It can be seen that the THD values are higher under lighter load conditions. Fig. 2-11(c) shows the $\mathrm{PF}$ curves under different $L_{s}$ values. It can be seen that a larger $L_{s}$ increases the input PF under lighter load conditions, but the PF could not be improved much under rated load. 

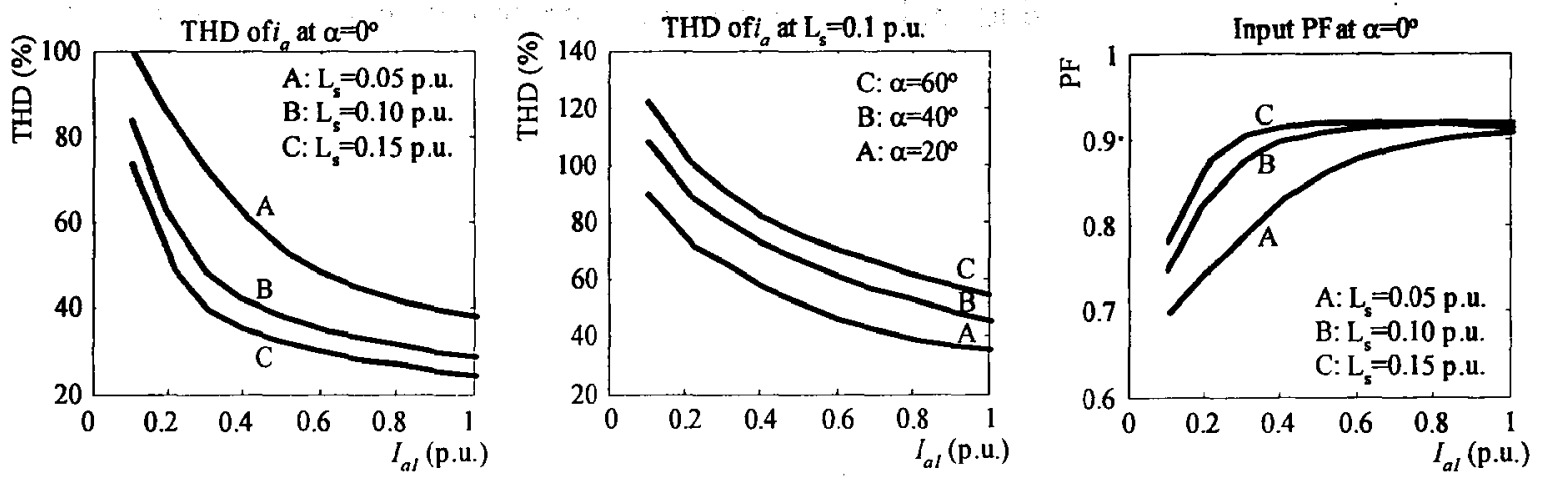

Fig. 2-11 THD and PF curves of the six-pulse SCR rectifier with capacitive loads.

\subsection{Summary}

This chapter presents the performances of the six-pulse SCR rectifier with inductive and capacitive loads. The following conclusions can be made:

- For the inductive load condition, the average load voltage is determined by the RMS value of the line-to-line supply voltage, the delay angle $\alpha$, and the line inductance $L_{s}$. The $L_{s}$ causes the commutation interval, which impacts the line current THD. The input PF is a function of the THD and the DPF.

- For the capacitive load condition, the line inductance $L_{s}$ and the load current amplitude influence the THD and the DPF of the line current. The input PF can be calculated based on the THD and the DPF.

- The line current THD of the six-pulse SCR rectifier with either the inductive or the capacitive load cannot meet the harmonic current requirement defined by IEEE Standard 519-1992. 


\section{Chapter 3 12-pulse SCR Rectifier}

As discussed in Chapter 2, the six-pulse SCR rectifier has a high line current THD, which does not meet the IEEE Standard 519-1992. Using multi-pulse (12-, 18- or 24pulse) SCR rectifiers, in which a phase-shifting transformer and a couple of six-pulse SCR rectifiers (at least two) are applied, could eliminate or decrease low-order harmonics, so as to reduce the line current THD.

This chapter presents the performance of the 12-pulse SCR rectifier with inductive and capacitive loads. The voltage and current waveforms are illustrated. Based on Fourier analysis and positive/negative sequence analysis, the principle of the harmonic elimination through the phase-shifting transformer is discussed. The line current THD and the input PF under different operating conditions are obtained by computer simulations.

\subsection{2-Pulse SCR Rectifier with Inductive Loads}

\subsubsection{Circuit Configuration}

Fig. 3-1(a) shows the configuration of the 12-pulse SCR rectifier with an inductive load. The rectifier is composed of one phase-shifting transformer and two identical sixpulse SCR rectifiers, which are discussed in Chapter 2. The transformer has one wyeconnected primary winding $\left(T_{p}\right)$, and two secondary windings $\left(T_{s l}\right.$ and $T_{s 2}$ ), feeding the two six-pulse SCR bridges. One of the secondary winding is wye-connected and the other is delta-connected. The different connection of the two windings results in a $30^{\circ}$ phase displacement between their line-to-line voltages (wye-connection is leading). The dc outputs of the two six-pulse bridges are connected in series to supply a dc load. Fig. 31(b) shows the simplified diagram, in which a dc current source $I_{L}$ replaces the inductive load in Fig. 3-1(a). 


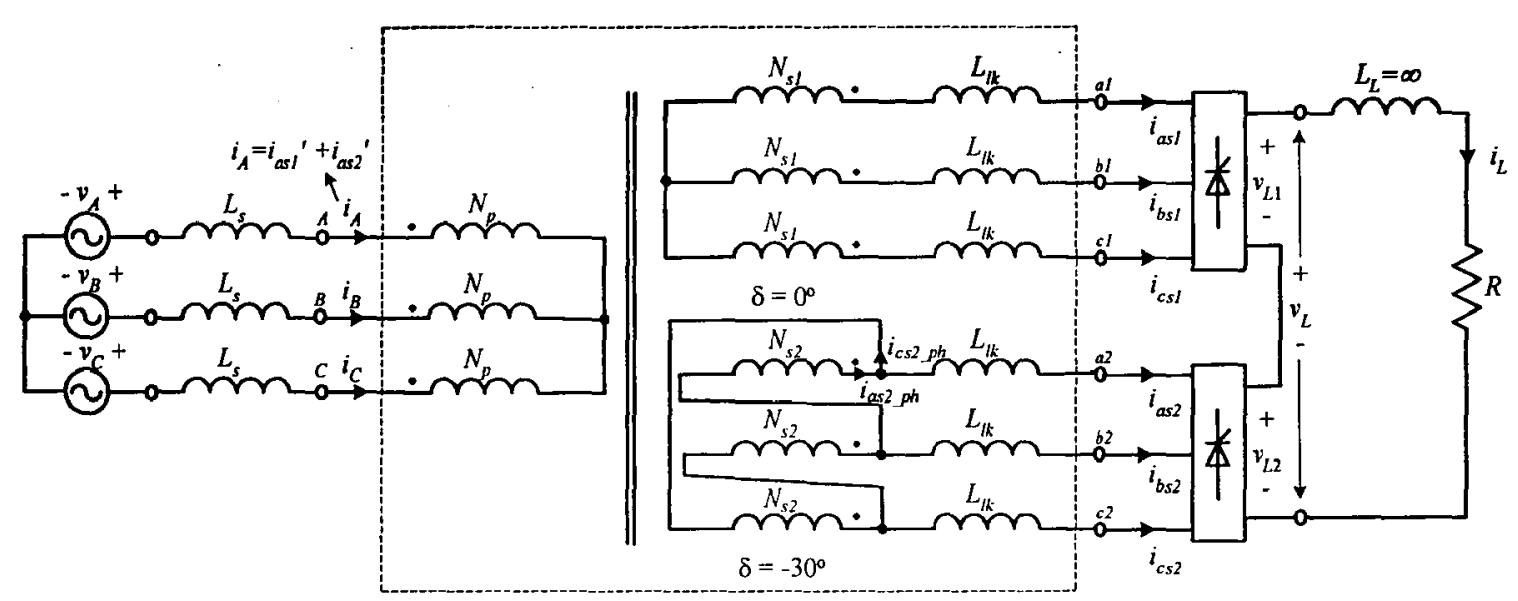

(a) 12-pulse SCR rectifier with an inductive load

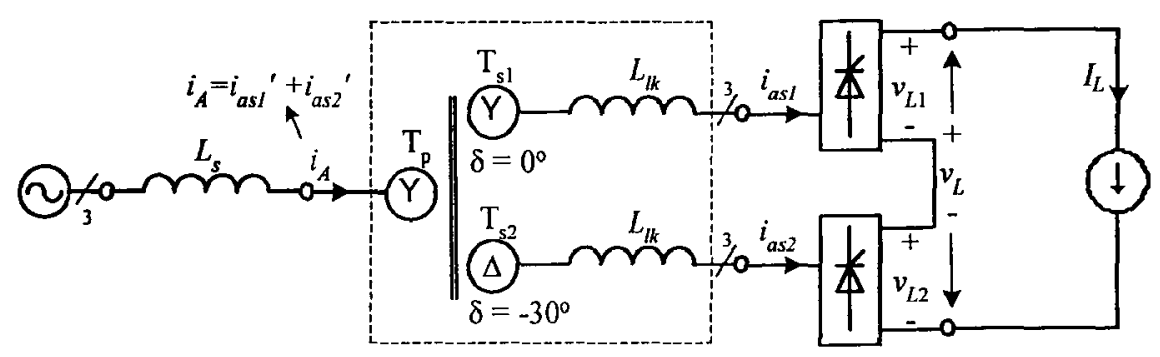

(b) Simplified diagram

Fig. 3-1 12-pulse SCR rectifier with an inductive load.

Note that in Fig. 3-1, $L_{s}$ represents the line inductance between the power supply and the phase-shifting transformer, and $L_{l k}$ stands for the total transformer leakage inductance, which is the summation of the primary winding's leakage inductance reflected to the secondary side and the secondary windings' leakage inductance.

The following equations apply to the phase-shifting transformer:

$$
\begin{gathered}
\frac{V_{A B}}{V_{a 1 b 1}}=\frac{V_{A B}}{V_{a 2 b 2}}=2, \\
\frac{N_{p}}{N_{s 1}}=2, \\
\frac{N_{p}}{N_{s 2}}=\frac{2}{\sqrt{3}}, \\
\delta=\angle V_{a 1 b 1}-\angle V_{A B}=0^{\circ},
\end{gathered}
$$




$$
\delta=\angle V_{a 2 b 2}-\angle V_{A B}=-30^{\circ},
$$

where $V_{A B}, V_{a 1 b 1}$ and $V_{a 2 b 2}$ are RMS line-to-line voltages of windings $T_{p}, T_{s 1}$ and $T_{s 2} ; N_{p}$, $N_{s 1}$ and $N_{s 2}$ are the number of turns in each phase of $T_{p}, T_{s 1}$ and $T_{s 2} ; \delta$ is the phase displacement of $v_{a \mid b 1}$ and $v_{a 2 b 2}$ with respect to $v_{A B}$. Since $T_{p}$ and $T_{s l}$ are both wyeconnected, there is no phase shift between their line-to-line voltages. $T_{s 2}$ is delta-connected, and thus its line-to-line voltages are $-30^{\circ}$ phase shifted with respect to the line-to-line voltages of $T_{p}$.

\subsubsection{Voltage and Current}

\section{1) Voltage and Current Waveforms}

Fig. 3-2 shows the waveforms of the 12-pulse SCR rectifier under ideal circuit condition ( $L_{s}=0$ and $L_{l k}=0$ ). Fig. 3-2(a) and Fig. 3-2(b) show the voltage waveforms at $\alpha=0$ and $\alpha=\pi / 3$, respectively, where $v_{L 1}$ and $v_{L 2}$ are the dc output voltages of the two six-pulse SCR bridges, and $v_{L}$ is the load voltage; i.e. $v_{L}=v_{L I}+v_{L 2}$. It can be noticed that both $v_{L I}$ and $v_{L 2}$ have six ripples in one supply voltage cycle. Since there is a $30^{\circ}$ phase shift between the transformer secondary windings' line-to-line voltages, $v_{L}$ has twelve ripples in one supply voltage cycle. The peak-to-peak amplitude of the ripple reaches the lowest, when the delay angle $\alpha$ is reduced to zero.

Fig. 3-2(c) to Fig. 3-2(f) show the current waveforms, where $i_{A}, i_{a s l}$ and $i_{a s 2}$ are line currents of windings $T_{p}, T_{s 1}$ and $T_{s 2} ; i_{a s 1}$ ' and $i_{a s 2}$ 'are values of $i_{a s l}$ and $i_{a s 2}$ reflected to the transformer primary side; $i_{L}$ is the load current with amplitude $I_{L} ; i_{S C R I_{-} l}$ and $i_{S C R Z_{-} l}$ are $S C R_{1}$ currents of the two SCR bridges, respectively. Fig. 3-2(d) shows that on the transformer secondary side, $i_{a s l}$ and $i_{a s 2}$ are identical, but with a $30^{\circ}$ phase shift. On the transformer primary side, $i_{a s 1}$ ' and $i_{a s 2}$ ' are in phase with $i_{A}$, and their summation is $i_{A}$. On both sides of the transformer, $i_{a s I}$ and $i_{a s l}$ ' are identical in shape, but have different amplitude due to the turns ratio; $i_{a s 2}$ and $i_{a s 2}$ ' have different shapes because of the $30^{\circ}$ phase shift. 


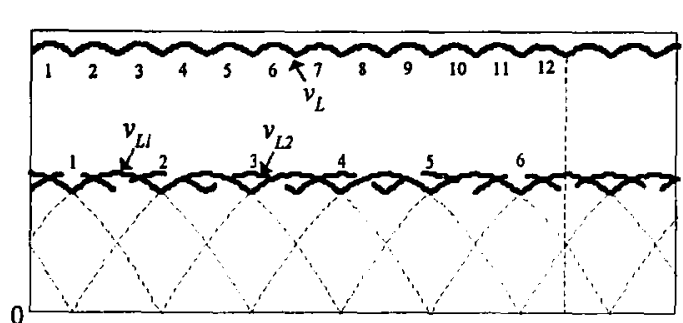

(a) Bridge output voltages at $\alpha=0$

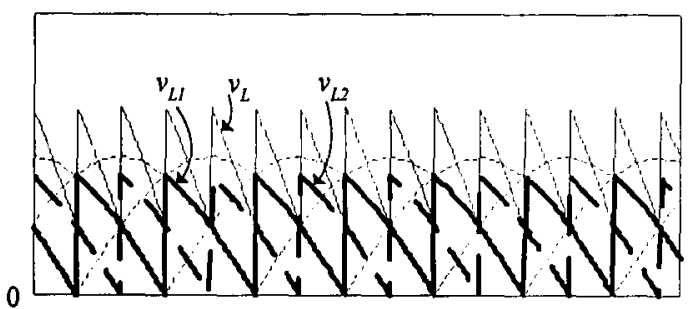

(b) Bridge output voltages at $\alpha=\pi / 3$

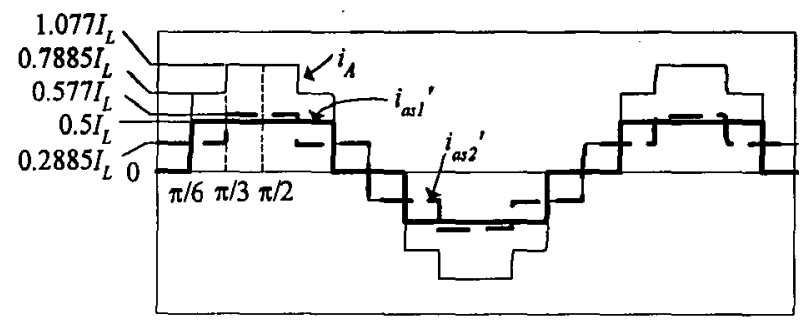

(c) Transformer primary side currents

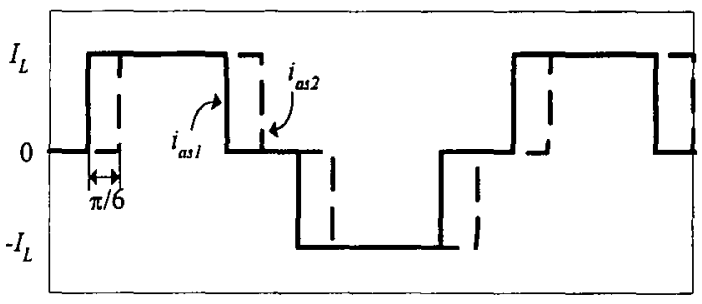

(d) Transformer secondary side currents
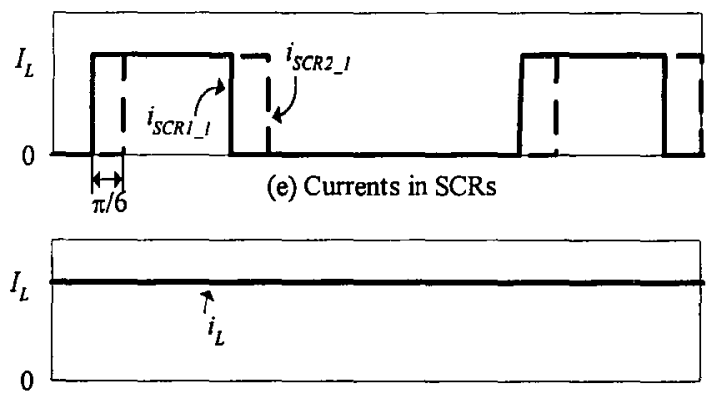

(f) Load current

Fig. 3-2 Voltage and current waveforms of the 12-pulse SCR rectifier with inductive loads.

\section{2) Average Load Voltage}

Under ideal circuit condition $\left(L_{s}=0\right.$ and $\left.L_{l k}=0\right)$, we have

$$
V_{L 1, \text { avg }}=V_{L 2, a v g}=1.35 V_{a 1 b 1} \cos \alpha \text {, }
$$

where $V_{L 1, \text { avg }}$ and $V_{L 2, \text { avg }}$ are the average values of $v_{L 1}$ and $v_{L 2}$, respectively. Fig. 3-3 shows the phasor diagram, in which $V_{L}$ is the phasor summation of $V_{L 1}$ and $V_{L 2}$. Thus $V_{L, a v g_{-} 12}$, the average value of $v_{L}$, can be calculated as follows:

$$
V_{L, a v g_{-} 12}=\left|V_{L}\right|=\left|V_{L 1}+V_{L 2}\right|=1.93 \times\left(1.35 V_{a 1 b 1} \cos \alpha\right) \text {. }
$$




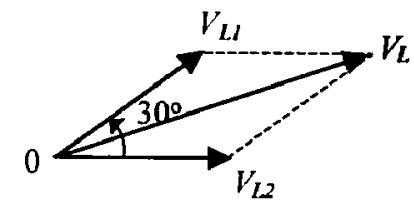

Fig. 3-3 Calculate the average load voltage of the 12-pulse SCR rectifier with inductive loads.

\section{3) RMS of the Transformer Primary Side Line Current}

Based on Fig. 3-2(c), the RMS value of $i_{A}$ can be calculated by:

$$
\begin{aligned}
& I_{A, R M S}=\sqrt{\frac{1}{2 \pi} \oint_{0}^{2 \pi}\left(i_{A}\right)^{2} d(\omega t)} \\
& =\sqrt{\frac{1}{2 \pi / 4}\left[\zeta_{0}^{\pi / 6}\left(0.2885 I_{L}\right)^{2}+\int_{\pi / 6}^{\pi / 3}\left(0.7885 I_{L}\right)^{2}+\int_{\pi / 3}^{\pi / 2}\left(1.077 I_{L}\right)^{2}\right]} \\
& =\sqrt{0.6216 I_{L}}=0.7884 I_{L}
\end{aligned}
$$

Compared to equation (2.12), this RMS value is lower than that of the six-pulse rectifier due to the harmonic elimination in the line current.

\subsubsection{Analysis of Harmonic Elimination}

1) Fourier Analysis

1a) Phase displacements of harmonics in the delta-connected winding line current when the transformer is $-30^{\circ}$ phase shifted.

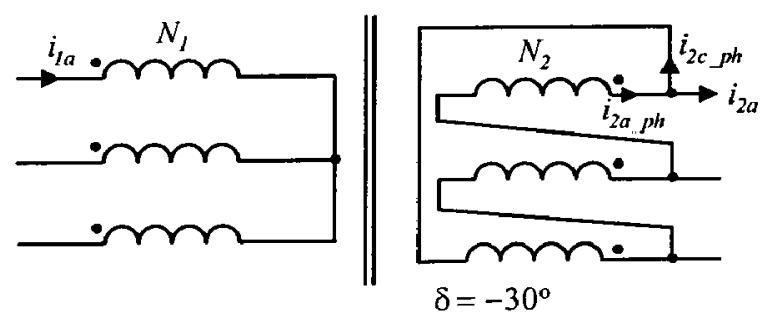

Fig. 3-4 Find the phase displacements of harmonics in the delta-connected winding line current when the transformer is $-30^{\circ}$ phase shifted. 
We'll use the circuit in Fig. 3-4 to find the phase displacements of harmonics in the delta-connected winding line current when the transformer is $-30^{\circ}$ phase shifted.

In Fig. 3-4, $i_{2 a_{-} p h}$ and $i_{2 c_{-} p h}$ are phase currents of the delta-connected secondary winding, and the line current $i_{2 a}=i_{2 a_{-} p h}-i_{2 c_{-} p h}$. If write the Fourier series of the primary line currents as follows:

$$
\begin{gathered}
i_{1 a}=I\left[\cos (\omega t)-\frac{1}{5} \cos 5(\omega t)+\frac{1}{7} \cos 7(\omega t)-\frac{1}{11} \cos 11(\omega t)+\frac{1}{13} \cos 13(\omega t)-\cdots\right], \\
i_{1 c}=I\left[\begin{array}{l}
\cos \left(\omega t+120^{\circ}\right)-\frac{1}{5} \cos 5\left(\omega t+120^{\circ}\right)+\frac{1}{7} \cos 7\left(\omega t+120^{\circ}\right) \\
-\frac{1}{11} \cos 11\left(\omega t+120^{\circ}\right)+\frac{1}{13} \cos 13\left(\omega t+120^{\circ}\right)-\cdots
\end{array}\right] .
\end{gathered}
$$

where $I$ is the amplitude of the fundamental component of $i_{1 a}$. Assume the transformer turns ratio is $1 / \sqrt{3}$, then we can write the Fourier series of $i_{2 a_{-} p h}$ and $i_{2 c_{-} p h}$ as:

$$
\begin{gathered}
i_{2 a_{-} p h}=\frac{I}{\sqrt{3}}\left[\cos (\omega t)-\frac{1}{5} \cos 5(\omega t)+\frac{1}{7} \cos 7(\omega t)-\frac{1}{11} \cos 11(\omega t)+\frac{1}{13} \cos 13(\omega t)-\cdots\right], \\
i_{2 c_{-} p h}=\frac{I}{\sqrt{3}}\left[\begin{array}{l}
\cos \left(\omega t+120^{\circ}\right)-\frac{1}{5} \cos 5\left(\omega t+120^{\circ}\right)+\frac{1}{7} \cos 7\left(\omega t+120^{\circ}\right) \\
-\frac{1}{11} \cos 11\left(\omega t+120^{\circ}\right)+\frac{1}{13} \cos 13\left(\omega t+120^{\circ}\right)-\cdots
\end{array}\right],
\end{gathered}
$$

Thus the Fourier series of $i_{2 a}$ is:

$$
i_{2 a}=i_{2 a_{-} p h}-i_{2 c_{-} p h}=I\left[\begin{array}{l}
\cos \left(\omega t-30^{\circ}\right)-\frac{1}{5} \cos \left(5 \omega t+30^{\circ}\right)+\frac{1}{7} \cos \left(7 \omega t-30^{\circ}\right) \\
-\frac{1}{11} \cos \left(11 \omega t+30^{\circ}\right)+\frac{1}{13} \cos \left(13 \omega t-30^{\circ}\right)-\cdots
\end{array}\right],
$$

Compare equation (3.9) with (3.13), we can see that the phase displacement $\delta=\angle i_{1 a n}-\angle i_{2 a n}(n=1,2,3, \ldots)$ is either $+30^{\circ}$ (for fundamental, $\left.7^{\text {th }}, 13^{\text {th }}, \ldots\right)$ or $-30^{\circ}$ (for $\left.5^{\text {th }}, 11^{\text {th }}, \ldots\right)$. This conclusion will be used in section $1 b$ ). 


\section{1b) Harmonic elimination from the 12-pulse SCR rectifier primary line current.}

For the 12-pulse SCR rectifier, based on Fig. 3-2(d) and equation (2.15), we can write the Fourier series of $i_{a s l}$ as:

$$
i_{a s 1}=1.1 I_{L}\left[\cos (\omega t)-\frac{1}{5} \cos 5(\omega t)+\frac{1}{7} \cos 7(\omega t)-\frac{1}{11} \cos 11(\omega t)+\frac{1}{13} \cos 13(\omega t)-\cdots\right] .
$$

Since $i_{a s 2}$ lags $i_{a s 1}$ by $30^{\circ}$, thus the Fourier series of $i_{a s 2}$ can be written as:

$$
i_{a s 2}=1.1 I_{L}\left[\begin{array}{l}
\cos \left(\omega t-30^{\circ}\right)-\frac{1}{5} \cos 5\left(\omega t-30^{\circ}\right)+\frac{1}{7} \cos 7\left(\omega t-30^{\circ}\right) \\
-\frac{1}{11} \cos 11\left(\omega t-30^{\circ}\right)+\frac{1}{13} \cos 13\left(\omega t-30^{\circ}\right)-\cdots
\end{array}\right] .
$$

Based on transformer turns ratio of $\frac{V_{A B}}{V_{a 1 b 1}}=\frac{V_{A B}}{V_{a 2 b 2}}=2$, the Fourier series of $i_{a s l}$ ' is:

$i_{a s 1}^{\prime}=\frac{1.1 I_{L}}{2}\left[\cos (\omega t)-\frac{1}{5} \cos 5(\omega t)+\frac{1}{7} \cos 7(\omega t)-\frac{1}{11} \cos 11(\omega t)+\frac{1}{13} \cos 13(\omega t)-\cdots\right]$

by using the conclusion in section 1a), we can write the Fourier series of $i_{\text {asz }}$ ' as:

$$
i_{a s 2}^{\prime}=\frac{1.1 I_{L}}{2}\left\{\begin{array}{l}
\cos \left[\left(\omega t-30^{\circ}\right)+30^{\circ}\right]-\frac{1}{5} \cos \left[5\left(\omega t-30^{\circ}\right)-30^{\circ}\right] \\
+\frac{1}{7} \cos \left[7\left(\omega t-30^{\circ}\right)+30^{\circ}\right]-\frac{1}{11} \cos \left[11\left(\omega t-30^{\circ}\right)-30^{\circ}\right] \\
+\frac{1}{13} \cos \left[13\left(\omega t-30^{\circ}\right)+30^{\circ}\right]-\cdots
\end{array}\right\} .
$$

Therefore, the Fourier series of the total line current $i_{A}$ can be obtained by:

$i_{A}=i_{a s 1}^{\prime}+i_{a s 2}^{\prime}=1.1 I_{L}\left[\cos (\omega t)-\frac{1}{11} \cos (11 \omega t)+\frac{1}{13} \cos (13 \omega t)-\frac{1}{23} \cos (23 \omega t)+\ldots\right]$,

in which the $5^{\text {th }}, 7^{\text {th }}, 17^{\text {th }}, 19^{\text {th }} \ldots$ order harmonics are zero, and the lowest order harmonic is the $11^{\text {th }}$. 


\section{2) Positive/Negative Sequence Analysis}

In a balanced three-phase system, the voltage sequence is defined to be positive when the three phase voltages reach their peak amplitudes in the order of A, B, C, and if in the order of A, C, B, the sequence is defined to be negative. Fig. 3-5 shows the phasor relation of the two kinds of voltage sequences. Similar definition applies to the three phase currents.

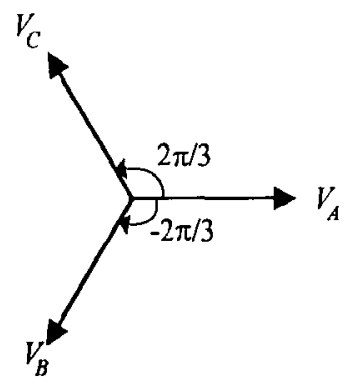

(a) positive sequence

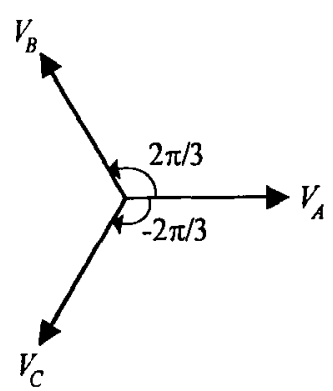

(b) negative sequence

Fig. 3-5 Positive and negative sequence three-phase voltage phasors.

For the three-phase positive sequence currents, we can write the equations as:

$$
\begin{gathered}
i_{a}=I_{m} \sin (\omega t), \\
i_{b}=I_{m} \sin \left(\omega t-\frac{2 \pi}{3}\right), \\
i_{c}=I_{m} \sin \left(\omega t+\frac{2 \pi}{3}\right) .
\end{gathered}
$$

For the three phase negative sequence currents, we can write the equations as:

$$
\begin{gathered}
i_{a}=I_{m} \sin (\omega t), \\
i_{b}=I_{m} \sin \left(\omega t+\frac{2 \pi}{3}\right), \\
i_{c}=I_{m} \sin \left(\omega t-\frac{2 \pi}{3}\right) .
\end{gathered}
$$

For the $5^{\text {th }}$ order harmonic currents, we can write the following equations:

$$
i_{a, 5 t h}=I_{5 m} \sin 5(\omega t)
$$




$$
\begin{aligned}
& i_{b, 5 t h}=I_{5 m} \sin 5\left(\omega t-\frac{2 \pi}{3}\right)=I_{5 m} \sin \left(5 \omega t+\frac{2 \pi}{3}\right), \\
& i_{c, 5 t h}=I_{5 m} \sin 5\left(\omega t+\frac{2 \pi}{3}\right)=I_{5 m} \sin \left(5 \omega t-\frac{2 \pi}{3}\right) .
\end{aligned}
$$

Hence the $5^{\text {th }}$ order harmonic currents are negative sequence currents. Similarly, we can prove that the $(5+6 k)^{\text {th }}(k=0,1,2, \ldots)$ order harmonics are negative sequence currents, and the $(7+6 k)^{\text {th }}(k=0,1,2, \ldots)$ order harmonics are positive sequence currents.

Passing through a phase-shifting transformer, if the positive sequence voltages or currents shift an angle $\Phi$, the negative sequence voltages or currents will shift an angle $-\Phi$. Fig. 3-6 shows an example of the phase-shift condition of the fundamental component $\left(I_{1}\right)$, one positive sequence component $\left(I_{7}\right)$ and one negative sequence component $\left(I_{5}\right)$ in a current. It can be seen that $I_{1}$ and $I_{7}$ are phase shifted in the same direction, but $I_{5}$ is shifted in the opposite direction.

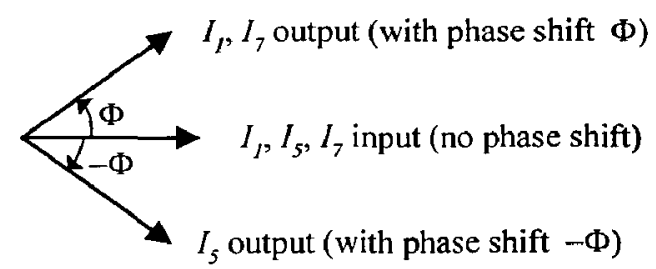

Fig. 3-6 Phase shift of positive sequence and negative sequence currents.

The fact that negative sequence voltages and currents are shifted in the opposite direction to positive sequence values when passing through a phase-shifting transformer makes it possible to cancel harmonics in pairs.

In each line current, if the fundamental component $I_{1}$ rotates at the angular frequency $\omega$, the $5^{\text {th }}$ order harmonic current $I_{5}$ will rotate at the angular frequency $5 \omega$, and the $7^{\text {th }}$ 
order harmonic current $I_{7}$ will rotate at the angular frequency $7 \omega$. As a result, when $I_{1}$ rotates $10^{\circ}, I_{5}$ will rotate $5 \times 10^{\circ}=50^{\circ}$ and $I_{7}$ will rotate $7 \times 10^{\circ}=70^{\circ}$.

In the 12-pulse rectifier, $i_{a s 1_{-} \mid s t}$ and $i_{a s 2_{2}{ }_{1 s t}}$ are the fundamental components of the two secondary line currents $i_{a s l}$ and $i_{a s 2}$, respectively. At any instant time, $i_{a s 2_{-} \mid s t} \operatorname{lags} i_{a s 1_{-} 1 s t}$ by $-30^{\circ}$; thus the $5^{\text {th }}$ order harmonic $i_{a s 2_{2} \text { Sth }}$ lags $i_{a s 1_{-} \text {Sth }}$ by $5 \times\left(-30^{\circ}\right)=-150^{\circ}$, and the $7^{\text {th }}$ order harmonic $i_{a s 2_{2} 7 t h}$ lags $i_{a s 1_{-} 7 h}$ by $7 \times\left(-30^{\circ}\right)=-210^{\circ}$. After being reflected to the primary side, $i_{a s l}$ ' has no phase shift with respect to $i_{a s l}$, but $i_{a s 2}$ has different phase shift in its reflected components: $i_{a s 2 \_ \text {st }}^{\prime}$ and all positive sequence components in $i_{a s 2}{ }^{\prime}$ are $30^{\circ}$ phase shifted with respect to those in $i_{a s 2}$, and all negative sequence components in $i_{a s 2}$, are $-30^{\circ}$ phase shifted. As a result, $i_{a s 2_{2} \mid 1 s t}^{\prime}$ becomes $-30^{\circ}+30^{\circ}=0^{\circ}$ lagging to $i_{a s 1_{1} 1 s t}^{\prime}$; $i_{a s 2_{-} \text {Sth }}$ is $-150^{\circ}-30^{\circ}=-180^{\circ}$ lagging to $i_{a s 1_{-} 5 t h} ;$ and $i_{a s 2_{-} 7 t h}^{\prime}$ is $-210^{\circ}+30^{\circ}=-180^{\circ}$ lagging to $i_{a s 1_{3} 7 t h}$. Since the amplitude of $i_{a s 1_{-} \text {sth }}$ and $i_{a s 2_{-} \text {sth }}$ are the same, the summation of them is zero. So do $i_{a s 1_{-} 7 t h}^{\prime}$ and $i_{a s 2 \_7 t h}^{\prime}$. Because the line current $i_{A}=i_{a s 1}^{\prime}+i_{a s 2}^{\prime}$, the $5^{\text {th }}$ and $7^{\text {th }}$ order harmonics are zero in $i_{A}$. The phenomenon applies to all harmonics in $i_{A}$ except the $(12 k \pm 1)^{\text {th }}$ order harmonics $(k=1,2,3, \ldots)$. Therefore, there is only the $(12 k \pm 1)^{t h}$ order harmonics $(k=1,2,3, \ldots)$ in $i_{A}$. Fig. 3-7 summarizes the phasor relation of the reflected harmonics being cancelled in the primary winding of the 12-pulse SCR rectifier.

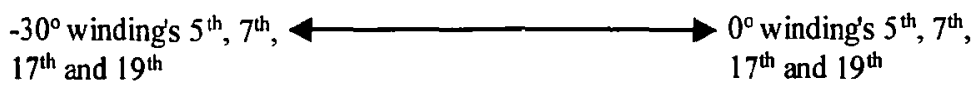

Fig. 3-7 Phasor relation of the reflected harmonics being cancelled in the primary winding of the 12-pulse SCR rectifier. 


\subsubsection{The Line Current THD and the Input PF}

Under ideal circuit conditions, the THD of the secondary and primary line currents of the 12-pulse SCR rectifier can be calculated based on equations (3.16), (3.17) and (3.18):

$$
\begin{gathered}
T H D_{i a s 1}=T H D_{i a s 2}=\frac{\sqrt{I_{a}{ }^{2}-I_{a 1}{ }^{2}}}{I_{a 1}}=\frac{\sqrt{I_{a 5}{ }^{2}+I_{a 7}{ }^{2}+I_{a 11}{ }^{2}+I_{a 13}{ }^{2}+\ldots}}{I_{a 1}}=31.1 \% \\
T H D_{i A}=\frac{\sqrt{I_{A}{ }^{2}-I_{A 1}{ }^{2}}}{I_{A 1}}=\frac{\sqrt{I_{A 11}{ }^{2}+I_{A 13}{ }^{2}+I_{A 23}{ }^{2}+I_{A 25}{ }^{2}+\ldots}}{I_{A 1}}=15.3 \%
\end{gathered}
$$

It can be seen that the THD of $i_{A}$ reduces about $50 \%$ compared to that of $i_{a s l}$ and $i_{a s 2}$, owing to the elimination of harmonics in $i_{A}$.

Fig. 3-8(a) shows the harmonic content of $i_{a s 1}$ and $i_{a s 2}$, where the $5^{\text {th }}$ and $7^{\text {th }}$ order harmonics have quite high amplitudes (about 20\% and 13\%), and the THD of $i_{a s l}$ or $i_{a s 2}$ is $28.73 \%$. Fig. 3-8(b) shows the harmonic content of $i_{A}$, in which the $5^{\text {th }}$ and $7^{\text {th }}$ order harmonics are zero and the THD is $12.89 \%$. The THD values are slightly lower than the result presented in equations (3.28) and (3.29) due to the inductance effect.

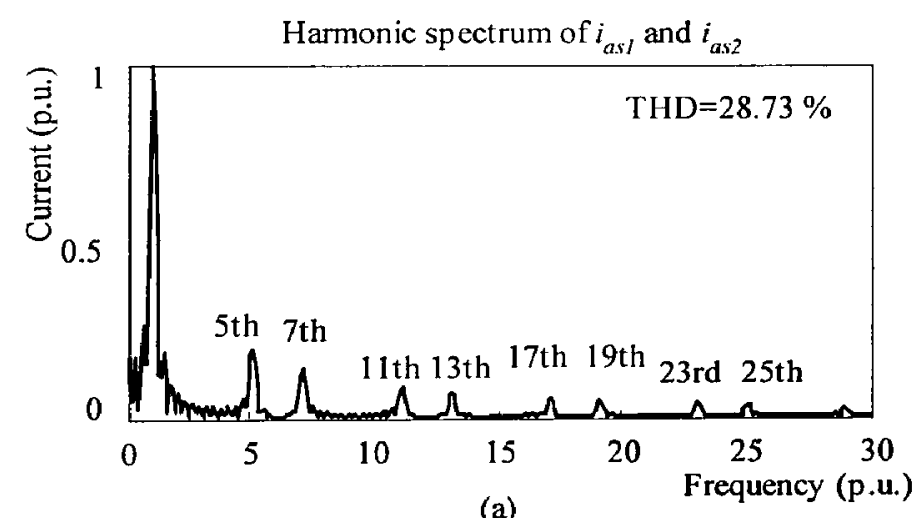

(a)

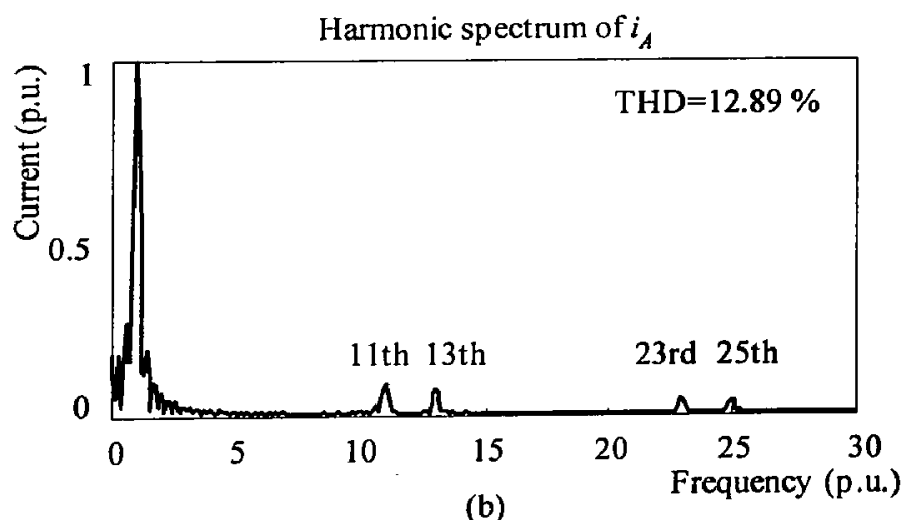

Fig. 3-8 Compare harmonic spectrum of $i_{a s l}, i_{a s 2}$ and $i_{A}$. 
Fig. 3-9 shows the THD of $i_{A}$ and the input PF of the 12-pulse SCR rectifier versus $I_{A 1}$ under different operating conditions. Compared to the plot of the six-pulse SCR rectifier shown in Fig. 2-7, the THD of the 12-pulse rectifier is reduced and the input PF is increased. However, the THD values still do not meet the harmonic current requirement of IEEE Standard 519-1992.
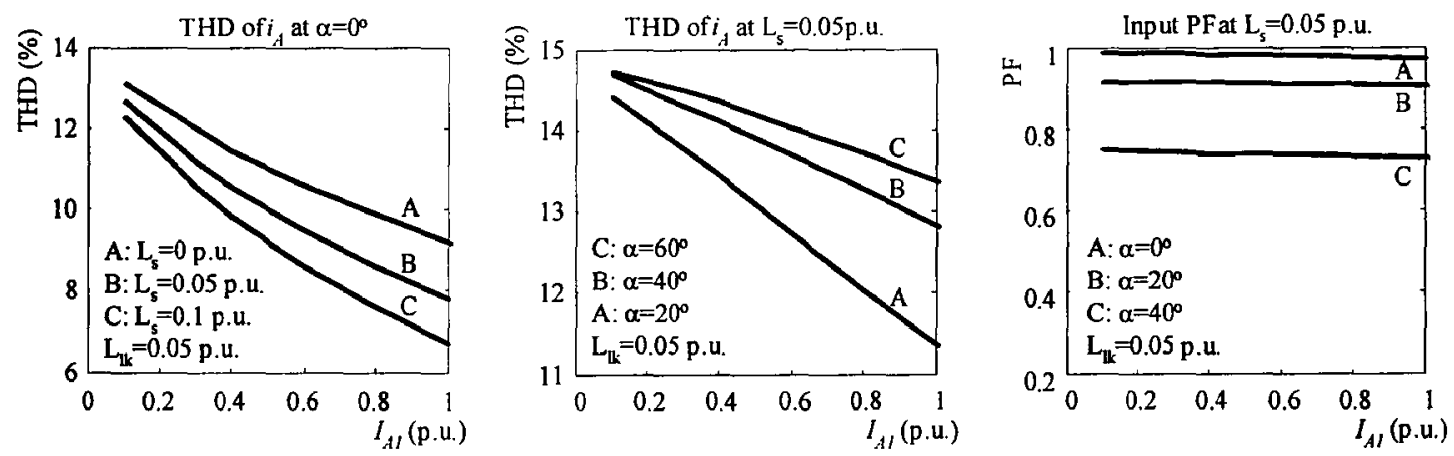

Fig. 3-9 THD and PF curves of the 12-pulse SCR rectifier with inductive loads.

\subsection{2-Pulse SCR Rectifier with Capacitive Loads}

\subsubsection{Circuit Configuration}

Fig. 3-10 shows the circuit configuration of the 12-pulse SCR rectifier with a capacitive load, in which the capacitive load is simplified to a dc voltage source $E$.

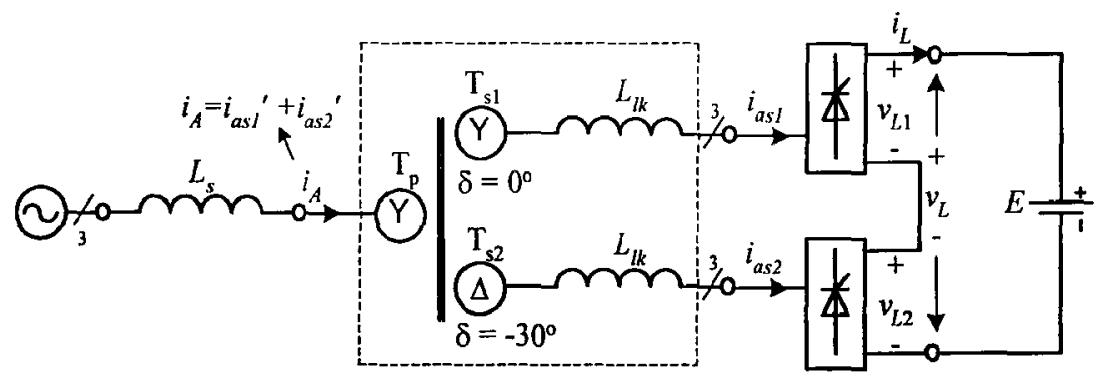

Fig. 3-10 12-pulse SCR rectifier with a capacitive load. 


\subsubsection{Current Waveforms}

Assuming no load is connected, the dc line-to-line voltage $v_{L_{-}} 0$ output by the rectifier is as shown in Fig. 3-11(a). The ripple frequency in $v_{L_{-}}$is twice that of the six-pulse rectifier. When apply $v_{L_{-} o}$ to the capacitive load, as long as $v_{L_{-}} 0$ is greater than $E$, and the related SCRs are triggered, the load current flows and causes energy exchanged through $L_{s}$. For each phase, the energy exchange happens for four times in every half cycle of the supply voltage, thus there are four humps in each half cycle of the line current as shown in Fig. 3-11(b), in which $i_{a s l}$ and $i_{a s 2}$ are the line currents of $T_{s l}$ and $T_{s 2}$, respectively. Compared to Fig. 2-10, the current ripple frequency is doubled. In Fig. 3-11(c), $i_{a s l}$ ' and $i_{a s 2}$ ' are $i_{a s 1}$ and $i_{a s 2}$ reflected to the transformer primary side, and their summation is the transformer primary line current $i_{A}$; i.e. $i_{A}=i_{a s 1}{ }^{\prime}+i_{a s 2}$ '. Since the dominant low-order harmonics, such as the $5^{\text {th }}$ and $7^{\text {th }}$, are eliminated as analyzed in Section 3.1.3, the shape of $i_{A}$ is more like sinusoidal.

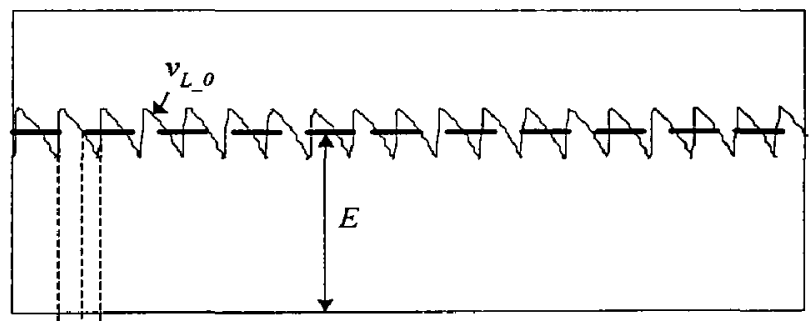

(a) Output voltage $v_{L_{-}}$with no load and the dc load voltage $E$

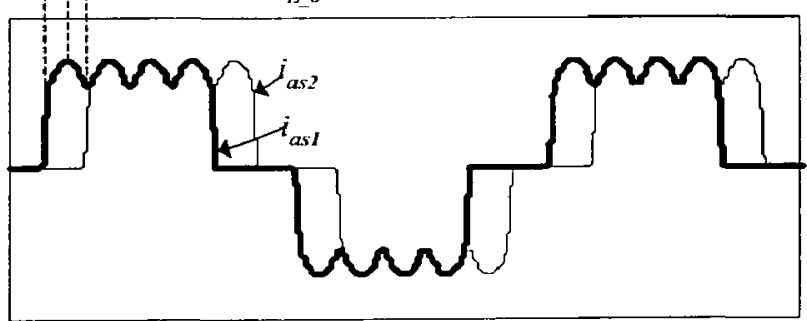

(b) transformer secondary side currents

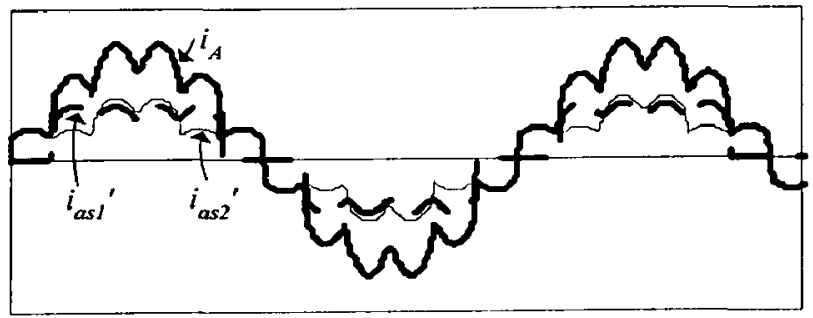

(c) transformer primary side currents

Fig. 3-11 Current waveforms of the 12-pulse SCR rectifier with capacitive loads. 
Fig. 3-12 shows the waveform and harmonic spectrum of $I_{A l}=1 \mathrm{pu}$ (rated load condition) and $I_{A I}=0.2 \mathrm{pu}$ (light load condition), respectively. It can be seen that the $i_{A}$ waveforms are quite different since the energy exchanged through $L_{s}$ varies when the load current changes. The $5^{\text {th }}, 7^{\text {th }}, 17^{\text {th }}$ and $19^{\text {th }}$ order harmonics are zero in $i_{A}$ owing to the phase-shifting transformer.
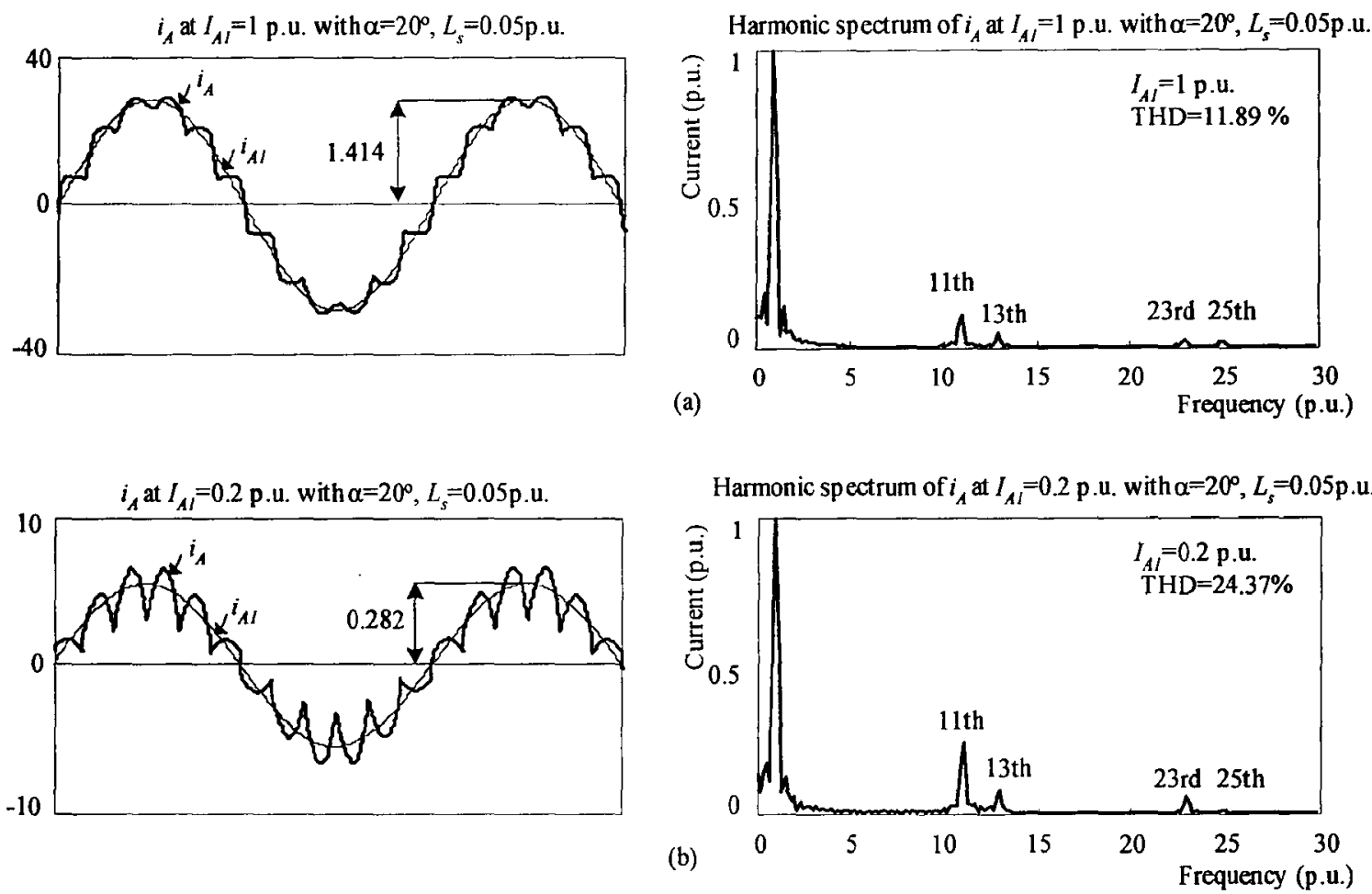

Fig. 3-12 Waveform and harmonic spectrum of $I_{A l}=1 \mathrm{pu}$ and $I_{A l}=0.2 \mathrm{pu}$.

\subsubsection{The Line Current THD and the Input PF}

Fig. 3-13(a) and Fig. 3-13 (b) show the THD of $i_{A}$ and the input PF versus $I_{A I}$ (from $0.1 \mathrm{pu}$ to $1 \mathrm{pu}$ ). The operating condition for Fig. 3-13(a) is $\alpha=0^{\circ}, L_{l k}=0.05 \mathrm{pu}$, and $L_{s}=0$, $0.05 \mathrm{pu}$ and $0.1 \mathrm{pu}$, respectively. As $I_{A l}$ or $L_{S}$ increases, the THD value decreases, and ranges from $21 \%$ to $7 \%$. For Fig. $3-13(\mathrm{~b})$, the operating condition is $L_{l k}=L_{s}=0.05 \mathrm{pu}$, and $\alpha=20^{\circ}, 40^{\circ}$ and $60^{\circ}$, respectively. As $\alpha$ decreases, the THD value decreases, and ranges from $41 \%$ to $13 \%$. Compared to Fig. 2-11, it can be seen that the THD values are reduced substantially because of the elimination of dominant low-order harmonics. 
In Fig. 3-13(c), a crossover occurs at approximately $I_{A I}=0.22$ pu. It's the boundary of the continuous and discontinuous current operation mode. When $I_{A l}$ is greater than $0.22 \mathrm{pu}$, the load current will be operating in the continuous mode.

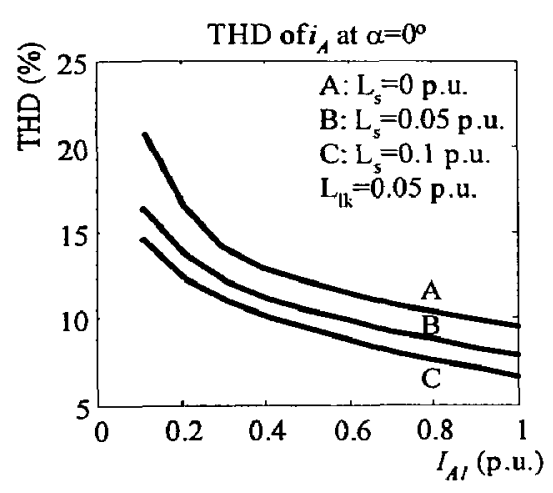

(a)

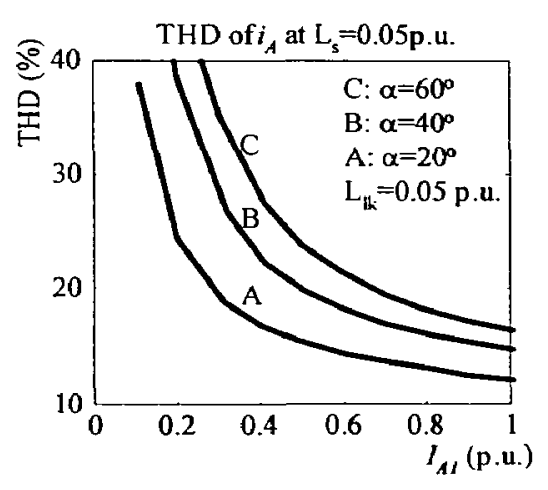

(b)

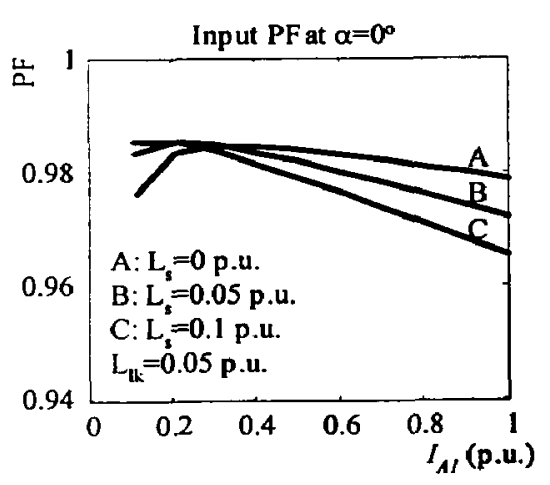

(c)

Fig. 3-13 THD and PF curves of the 12-pulse SCR rectifier with capacitive loads. 


\subsection{Summary}

This chapter presents the performance of the 12-pulse SCR rectifier with inductive and capacitive loads. The following conclusions can be made:

- The principle of the harmonic elimination through the phase-shifting transformer can be illustrated by Fourier analysis and the positive/negative sequence analysis.

- For the inductive load condition, the ripple in the dc load voltage has a doubled frequency and reduced peak-to-peak amplitude compared to the six-pulse rectifier. The RMS value of the line current is lower than that of the six-pulse rectifier due to the harmonic elimination.

- For both of the load conditions, the line current THD values are lower than those of the six-pulse rectifier, because the dominant low-order harmonics are eliminated. The input $\mathrm{PF}$ of the 12-pulse rectifier is improved.

- The line current THD of the 12-pulse SCR rectifier with either the inductive or the capacitive load still cannot meet the harmonic current requirement in IEEE Standard 519-1992. 


\section{Chapter 4 18-pulse SCR Rectifier}

Since the line current THD of the 12-pulse SCR rectifier does not meet the harmonic current requirement in IEEE Standard 519-1992, it is required to eliminate more loworder harmonic currents. The 18-pulse SCR rectifier is an option to improve the line current THD.

This chapter presents the performance of the 18-pulse SCR rectifier with inductive and capacitive loads. The voltage and current waveforms are shown. The harmonic spectrum of the line current and the reason of harmonic elimination are presented. The line current THD and the input PF are discussed.

\subsection{8-Pulse SCR Rectifier with Inductive Loads}

\subsubsection{Circuit Configuration}

Fig. 4-1 shows the simplified diagram of the 18-pulse SCR rectifier with an inductive load. The phase-shifting transformer in the rectifier has three secondary windings $\left(T_{s l}, T_{s 2}\right.$ and $T_{s 3}$ ), feeding three six-pulse SCR bridges. Different from the $30^{\circ}$ phase shift in the 12-pulse rectifier, the phase shift between any two adjacent windings in the 18-pulse rectifier is $20^{\circ}$, which is realized by means of the zigzag-winding connection. The details of the zigzag transformer will not be discussed in this thesis. As shown in Fig. 4-1, the phase displacement $\delta$ of the line-to-line voltages of $T_{s 1}, T_{s 2}$ and $T_{s 3}$ with respect to the line-to-line voltage of the primary winding $T_{p}$ is $0^{\circ},-20^{\circ}$ and $-40^{\circ}$, respectively. Other arrangements for $\delta$, such as $-20^{\circ}, 0^{\circ}$ and $20^{\circ}$, are also possible. The turns ratio will be selected to make the line-to-line voltage of each secondary winding one third of the lineto-line voltage of the primary winding. 


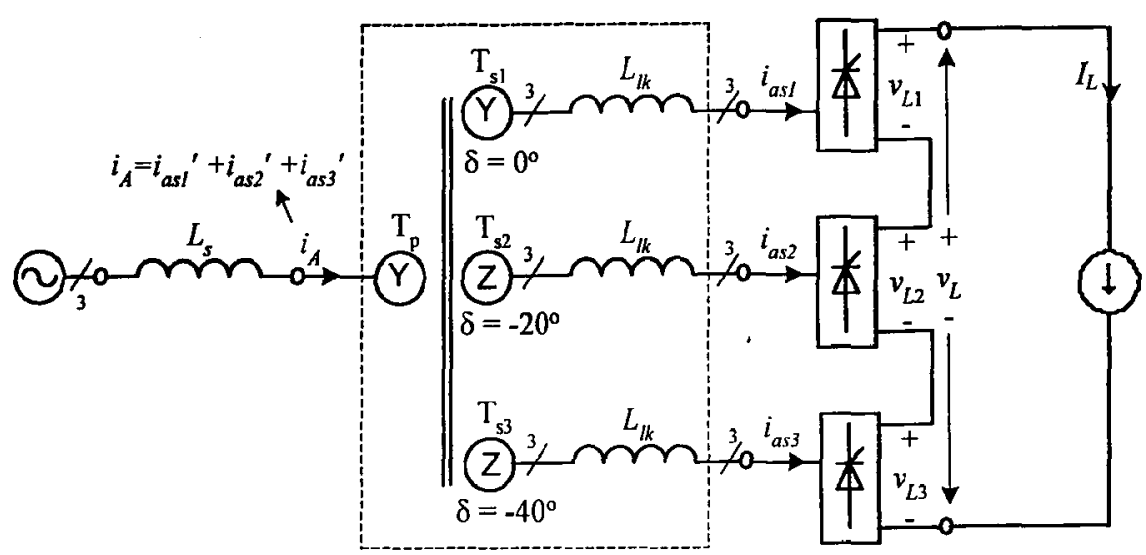

Fig. 4-1 18-pulse SCR rectifier with an inductive load.

\subsubsection{Voltage and Current Waveforms}

Fig. 4-2 shows the voltage and current waveforms of the 18-pulse SCR rectifier with inductive loads under the condition of $\alpha=30^{\circ}, I_{A l}=1 \mathrm{pu}, L_{s}=0$ and $L_{l k}=0.05 \mathrm{pu}$. From Fig. 4-2(c), it can be seen that the transformer secondary currents $i_{a s l}, i_{a s 2}$ and $i_{a s 3}$ are $20^{\circ}$ phase shifted between any adjacent two. Fig. 4-2(b) shows the waveforms of $i_{a s l}, i_{a s 2}{ }^{\prime}$ and $i_{a s 3}$, which are $i_{a s l}, i_{a s 2}$ and $i_{a s 3}$ reflected to the transformer primary side. Their fundamental components are in phase. The shapes of $i_{a s 2}$ ' and $i_{a s 3}$ ' are the result of the zigzag connections. The summation of $i_{a s i}{ }^{\prime}, i_{a s 2}$ ' and $i_{a s 3}{ }^{\prime}$ is the line current $i_{A}$, which is shown in Fig. 4-1(a). Fig. 4-2(e) shows the harmonic content of $i_{A}$, in which the $5^{\text {th }}, 7^{\text {th }}$, $11^{\text {th }}$ and $13^{\text {th }}$ order harmonics are eliminated; thus the lowest order harmonic is the $17^{\text {th }}$ and the THD is $7.45 \%$.

In Fig. 4-2(d), $v_{L l}, v_{L 2}$ and $v_{L 3}$ are output voltages of the three six-pulse SCR bridges respectively. Their waveforms are the same as that of the typical six-pulse bridge, but are $20^{\circ}$ phase shifted. Their summation is the load voltage $v_{L}$, in which the ripple frequency is tripled compared to $v_{L 1}, v_{L 2}$ and $v_{L 3}$. Hence the 18-pulse rectifier requires a smaller dc choke to obtain a ripple free dc load current compared to the 12-pulse rectifier. 


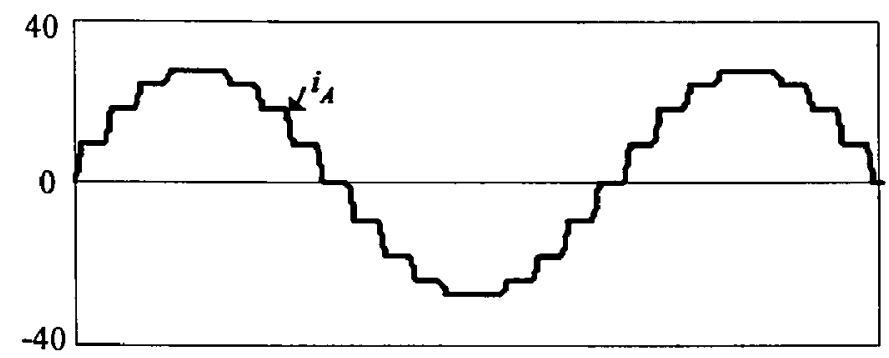

(a) Transformer primary side current $i_{A}$

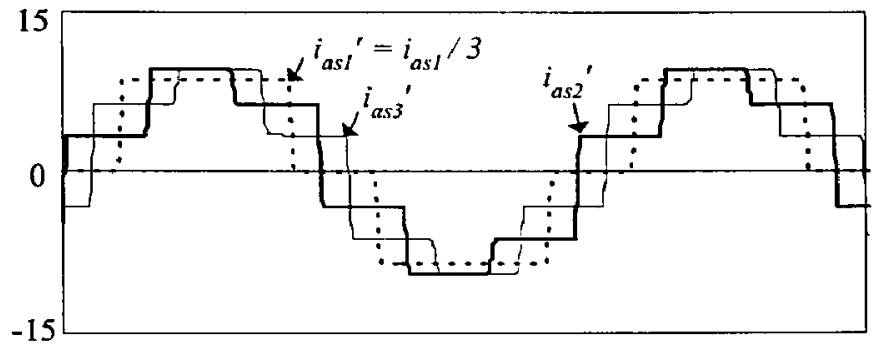

(b) Transformer primary side currents $i_{a s l}{ }^{\prime}, i_{a s 2}{ }^{\prime}$ and $i_{a s 3}{ }^{\prime}$

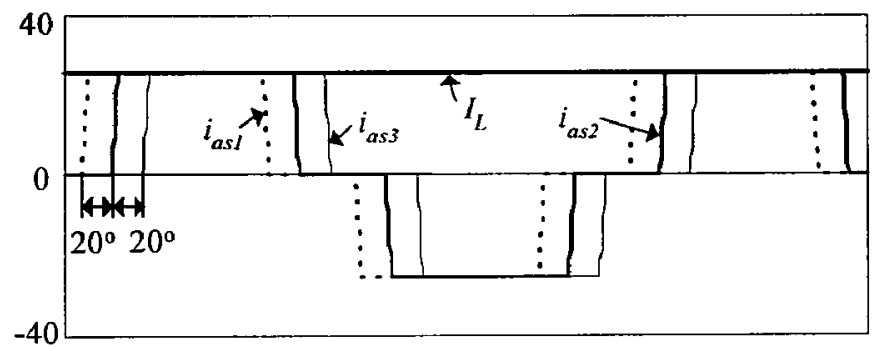

(c) Transformer secondary side currents $i_{a s l}, i_{a s 2}$ and $i_{a s 3}$ and the load current $I_{L}$

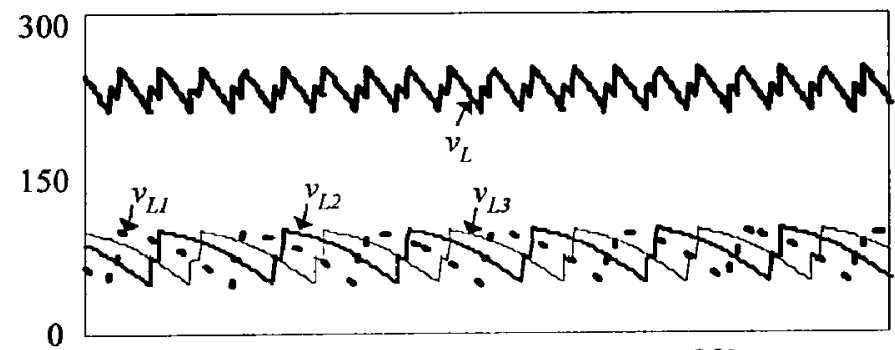

(d) Bridge output voltages at $\alpha=30^{\circ}$

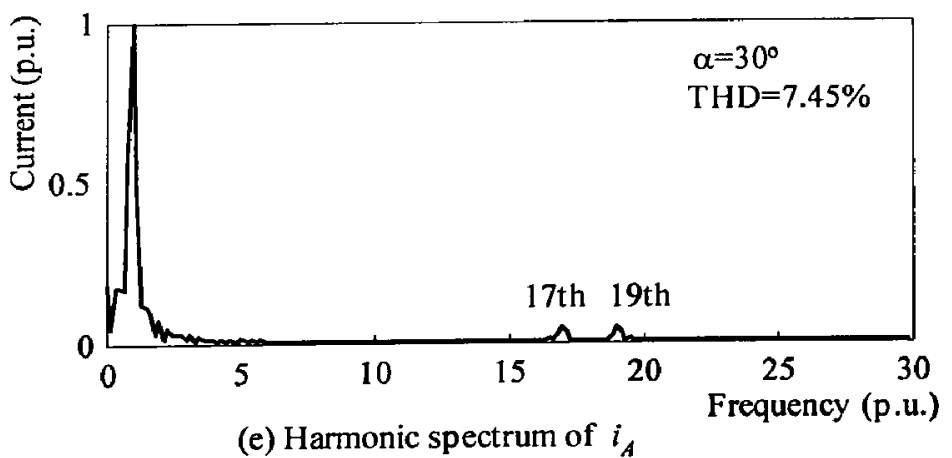

Fig. 4-2 Waveforms of the I8-pulse SCR rectifier with inductive loads. 


\subsubsection{Analysis of Harmonic Elimination}

It's convenient to use positive / negative sequence analysis to explain the reason of harmonic elimination in the 18-pulse rectifier. Since the fundamental component of $i_{a s 2}$ lags $i_{a s l}$ by $-20^{\circ}$, the $5^{\text {th }}$ order harmonic of $i_{a s 2}$ lags the $5^{\text {th }}$ order harmonic of $i_{a s l}$ by $5 \times\left(-20^{\circ}\right)=-100^{\circ}$. After the two $5^{\text {th }}$ order harmonics being reflected to the transformer primary side, the phase displacement between them becomes $-120^{\circ}$. Similarly it can be obtained that the phase displacement between the reflected $5^{\text {th }}$ order harmonics of $i_{a s l}$ and $i_{a s 3}$ is $-240^{\circ}$. Hence the phasor summation of the three reflected $5^{\text {th }}$ order harmonics of the three secondary windings is zero in $i_{A}$. The same condition happens to all harmonics in $i_{A}$, except the fundamental component and the $(18 k \pm 1)^{t h}$ order harmonics $(k=1,2,3 \ldots)$. Fig. 4-3 summarizes the phasor relation of the reflected harmonics being cancelled in the primary winding of the 18 -pulse SCR rectifier.

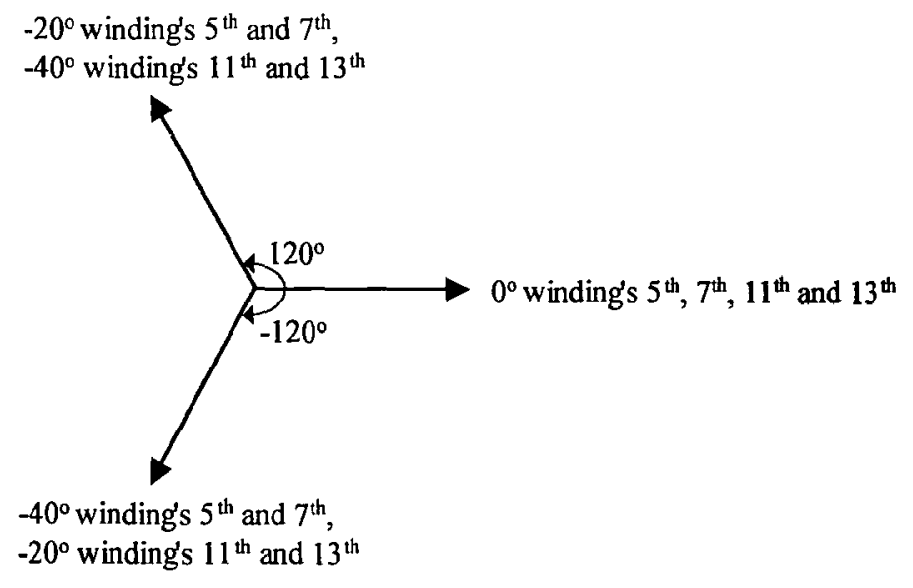

Fig. 4-3 Phasor relation of the reflected harmonics being cancelled in the primary winding of the 18 -pulse SCR rectifier.

\subsubsection{The Line Current THD and the Input PF}

Fig. 4-4 shows the line current THD and the input PF of the 18-pulse SCR rectifier versus $I_{A I}$. Compared to the 12-pulse rectifier, the THD values of the 18-pulse rectifier are reduced, but the input PF is almost the same. When $\alpha=0^{\circ}$ and $I_{A 1}>0.5 \mathrm{pu}$, the THD values are less than 5\%, which satisfy the IEEE Standard 519-1992. 

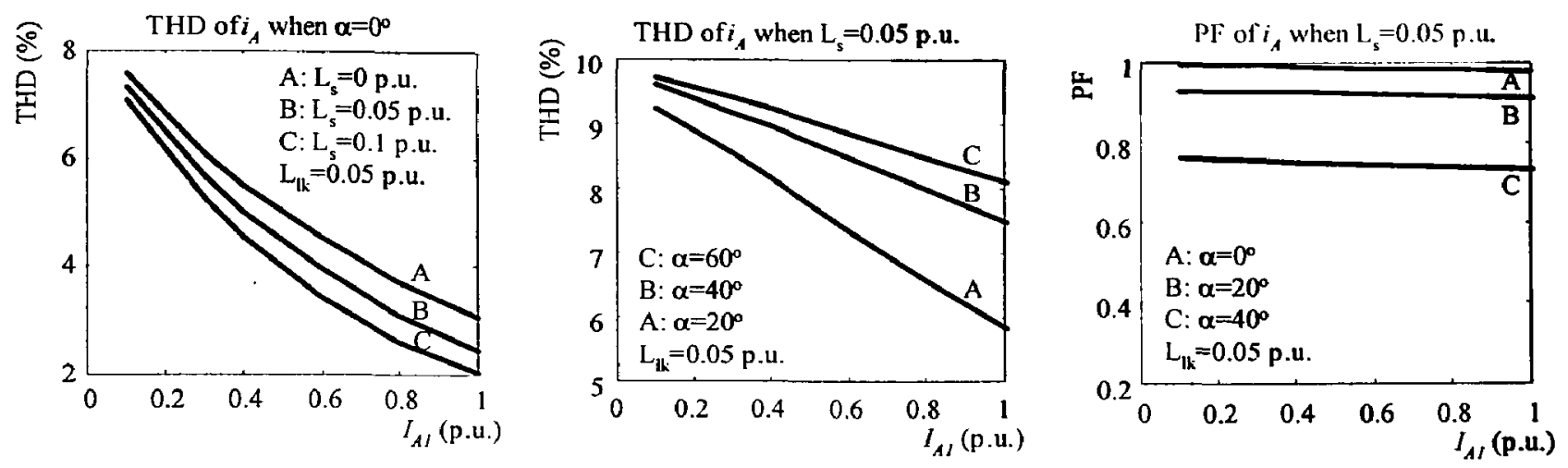

Fig. 4-4 THD and PF curves of the 18-pulse SCR rectifier with inductive loads.

\subsection{8-Pulse SCR Rectifier with Capacitive Loads}

\subsubsection{Circuit Configuration}

Fig. 4-5 shows the simplified diagram of the 18-pulse SCR rectifier with a capacitive load. Since three typical six-pulse bridges are connected in series, the circuit is able to handle higher voltages than the 12-pulse rectifier.

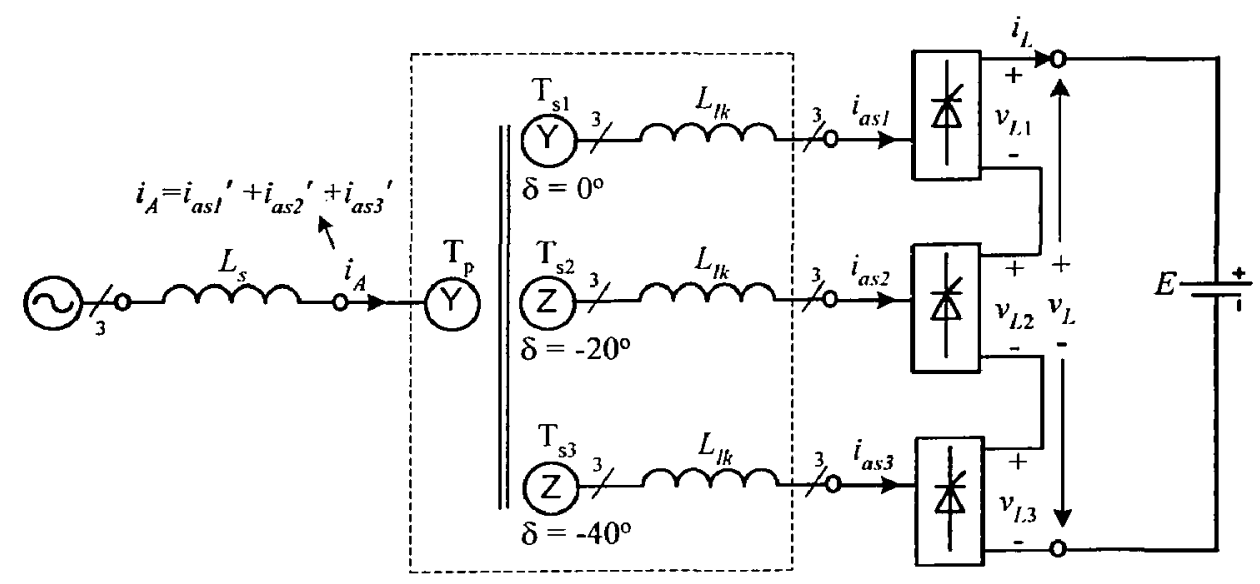

Fig. 4-5 18-pulse SCR rectifier with a capacitive load.

\subsubsection{Current Waveforms}

Fig. 4-6 shows the current waveforms under the condition of $\alpha=30^{\circ}, I_{A I}=1 \mathrm{pu}, L_{s}=0$ and $L_{l k}=0.05 \mathrm{pu}$. From Fig. $4-6(\mathrm{a})$, it can be seen that $i_{A}$ looks closer to a sinusoid, because more low-order harmonics are eliminated. In Fig. 4-6(e), the lowest order harmonic in $i_{A}$ is the $17^{\text {th }}$, and the THD of $i_{A}$ is $7.92 \%$. In Fig. 4-6(b), the shapes of $i_{a s 2}$ ' 
and $i_{a s 3}$ ' are the result of the zigzag connections. The six small ripples on the positive half cycle of each secondary line current are shown in Fig. 4-6(c). It's the result of the involvement of the three transformer secondary windings and the three six-pulse SCR bridges.

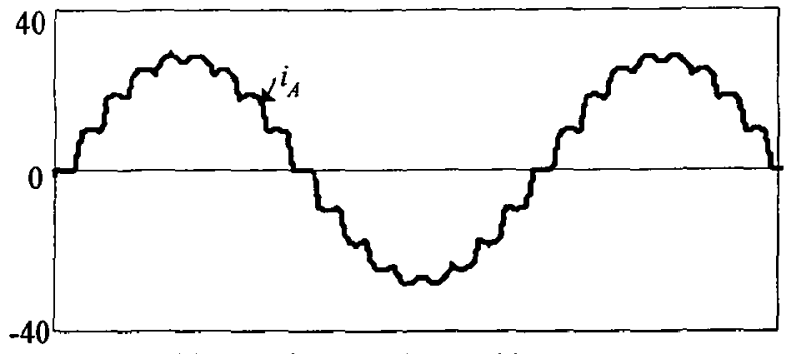

(a) Transformer primary side current $i_{A}$
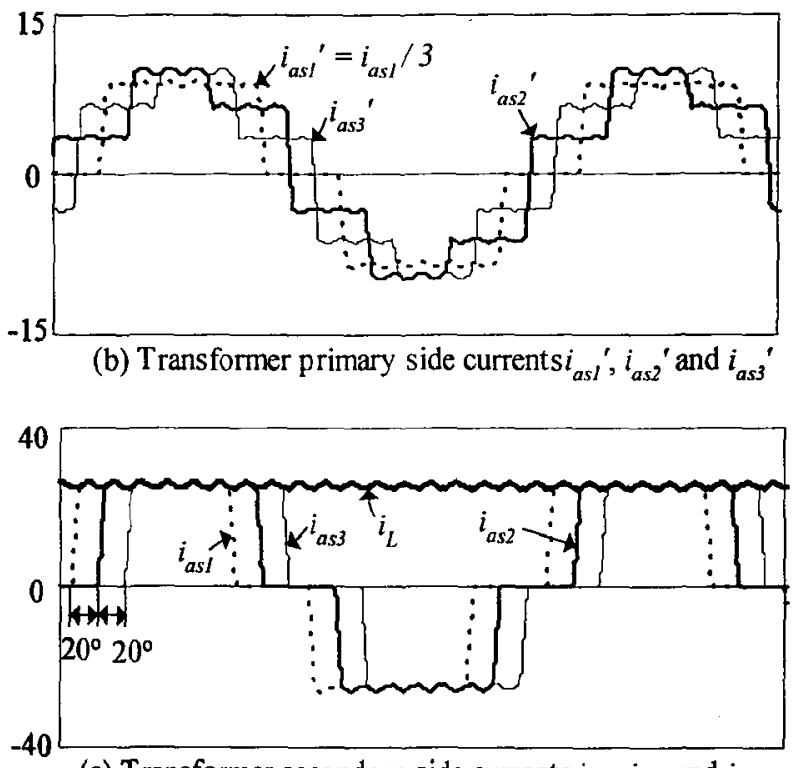

(c) Transformer secondary side currents $i_{a s /}, i_{a s 2}$ and $i_{a s 3}$ and the load current $i_{L}$

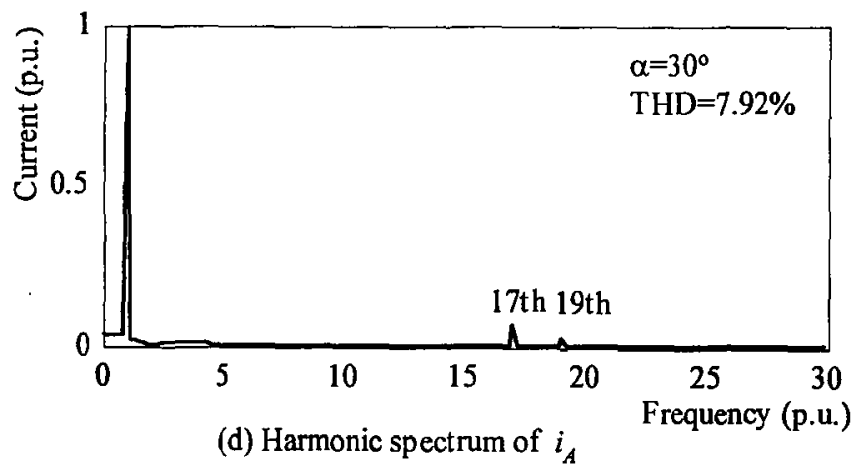

Fig. 4-6 Waveforms of the 18-pulse SCR rectifier with capacitive loads. 


\subsubsection{The Line Current THD and the Input PF}

Fig. 4-7 shows the waveform and the harmonic spectrum of $i_{A}$, when $\alpha=20^{\circ}, I_{A I}=1$ $\mathrm{pu}$ (rated load condition) and $I_{A I}=0.2 \mathrm{pu}$ (light load condition). It can be seen that the $5^{\text {th }}$, $7^{\text {th }}, 11^{\text {th }}$ and $13^{\text {th }}$ order harmonics are eliminated, and the amplitude of the current ripple is substantially reduced, compared to the 12-pulse SCR rectifier. The THD values are $6.036 \%\left(I_{A I}=1 \mathrm{pu}\right)$ and $12.5 \%\left(I_{A l}=0.2 \mathrm{pu}\right)$, which are less than those of the 12-pulse rectifier under the same conditions.
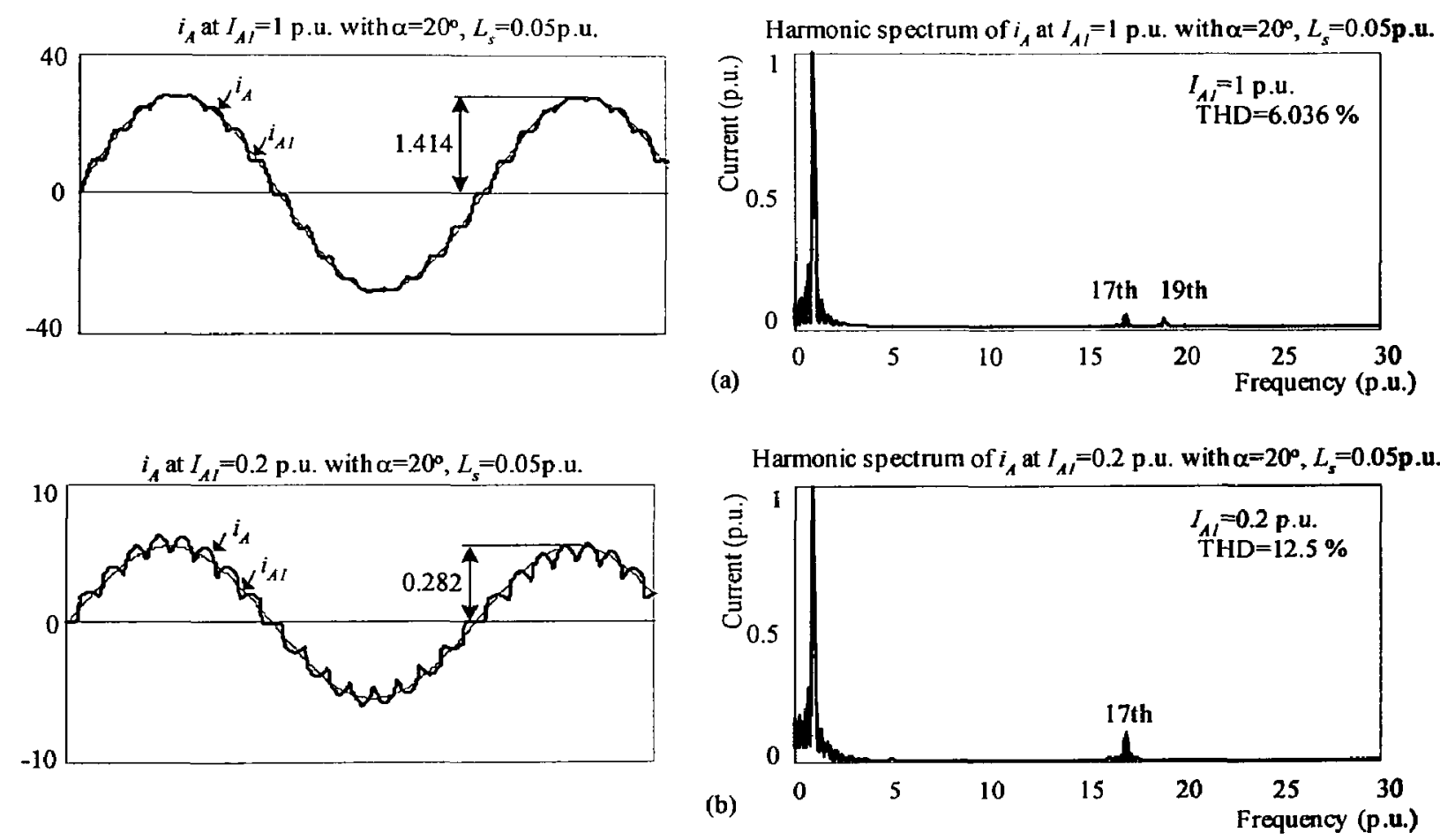

Fig. 4-7 Current waveform and harmonic spectrum of $I_{A l}=1 \mathrm{pu}$ and $I_{A I}=0.2 \mathrm{pu}$.

Fig. 4-8(a) and Fig. 4-8(b) show the $i_{A}$ THD at different value of $L_{s}, I_{A l}$ and $\alpha$. Under the condition of $\alpha=0^{\circ}$ and $I_{A P}>0.5 \mathrm{pu}$, the THD values are less than 5\%. Fig. 4-8(c) shows the input PF of the 18-pulse SCR rectifier with capacitive loads. The values are improved slightly compared to that of the 12-pulse rectifier. Since three transformer secondary windings are connected in series, the total transformer leakage inductance becomes larger. Therefore the load current operates in the continuous mode. 


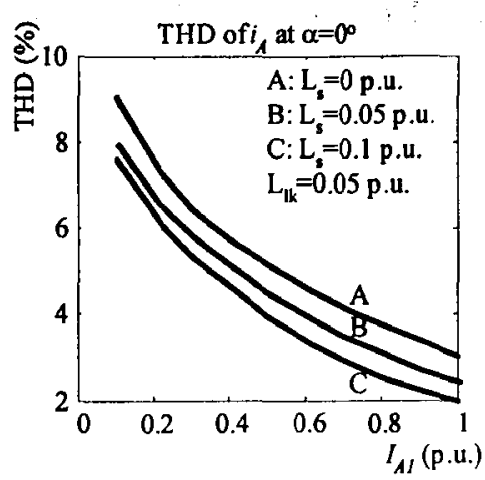

(a)

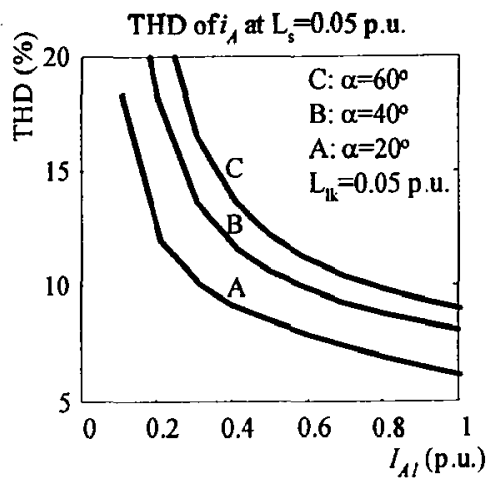

(b)

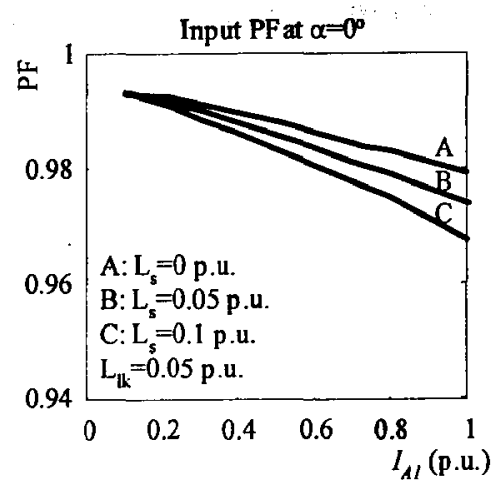

(c)

Fig. 4-8 THD and PF curves of the 18-pulse SCR rectifier with capacitive loads.

\subsection{Summary}

This chapter presents the performance of the 18-pulse SCR rectifier with the inductive and capacitive loads. The following conclusions can be made:

- The principle of the harmonic elimination can be applied to the 18-pulse rectifier.

- For the inductive load condition, the ripple amplitude in the dc load voltage is reduced, and the ripple frequency is tripled compared to that of the six-pulse rectifier.

- For the capacitive load condition, since the leakage inductance of the three transformer secondary windings is in-series, the load current operates in the continuous mode.

- For the two load conditions, the THD values are substantially reduced, because more low-order harmonics are eliminated.

- For the two load conditions, when the delay angle $\alpha=0^{\circ}$ and $I_{A P}>0.5 \mathrm{pu}$, the line current THD is less than 5\%, which meets the harmonic current requirement of IEEE Standard 519-1992. 


\section{Chapter 5 24-pulse SCR Rectifier}

In order to further reduce the line current THD and handle higher voltages, 24-pulse SCR rectifiers are employed. This chapter presents the performance of the 24-pulse SCR rectifier with inductive and capacitive loads. The line current THD and the input PF of the 24-pulse rectifier are discussed.

\subsection{4-Pulse SCR Rectifier with Inductive Loads}

\subsubsection{Circuit Configuration}

Fig. 5-1 shows the simplified diagram of the 24-pulse SCR rectifier with an inductive load. The phase-shifting transformer in the diagram has four secondary windings, feeding four six-pulse SCR bridges. Different from the $20^{\circ}$ phase-shifted zigzag windings in the 18-pulse rectifier, the transformer secondary windings in the 24-pulse rectifier are $15^{\circ}$ phase shifted. As shown in Fig. 5-1, the phase displacement $\delta$ of the secondary line-toline voltages with respect to the primary line-to-line voltage are $0^{\circ},-15^{\circ},-30^{\circ}$ and $-45^{\circ}$. Other arrangements, such as $-15^{\circ}, 0^{\circ}, 15^{\circ}$, and $30^{\circ}$, are also practicable. The winding turns ratios are selected to make the line-to-line voltage of each secondary winding one forth of the line-to-line voltage of the primary winding. 


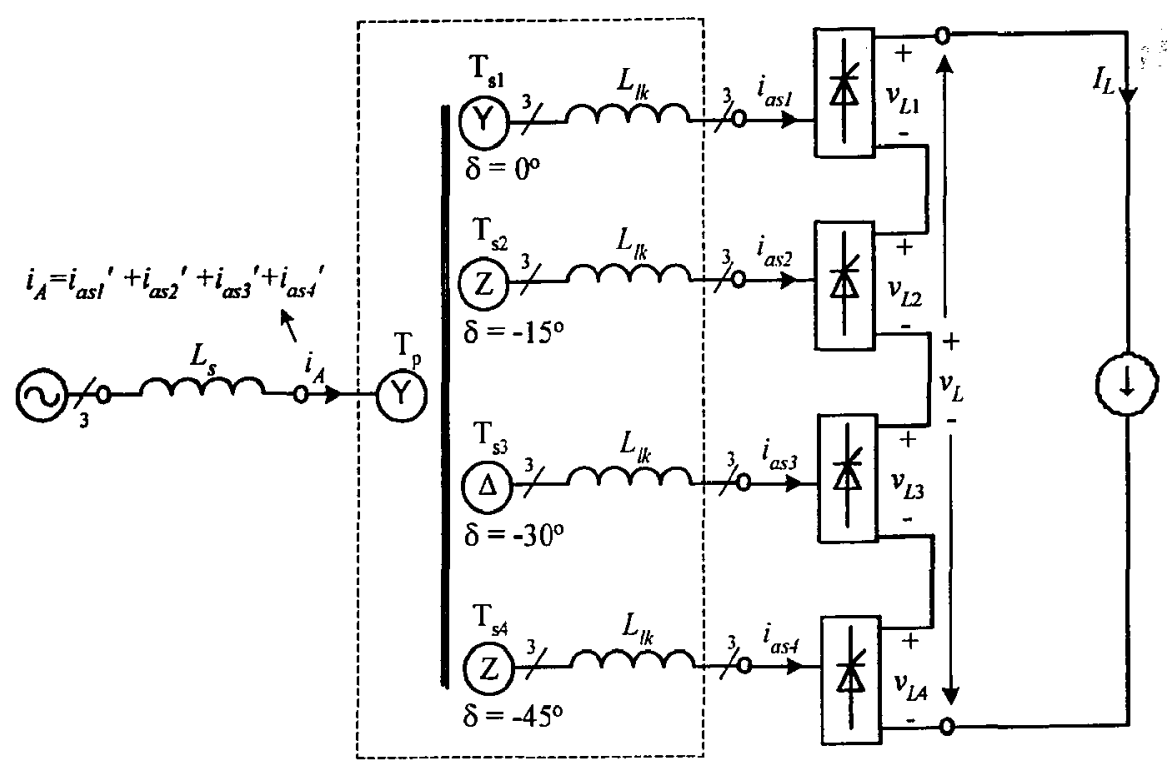

Fig. 5-1 24-Pulse SCR rectifier with an inductive load.

\subsubsection{Voltage and Current Waveforms}

Fig. 5-2 shows the voltage and current waveforms under the condition of $\alpha=30^{\circ}, I_{A I}$ $=1 \mathrm{pu}, L_{s}=0$ and $L_{l k}=0.05 \mathrm{pu}$. Fig. $5-2$ (c) shows the transformer secondary line currents $i_{a s 1}, i_{a s 2}, i_{a s 3}$ and $i_{a s 4}$, which are identical in waveforms, but are $15^{\circ}$ phase shifted between each other. Reflecting these currents to the transformer primary side, we get $i_{\text {asl }}$ ', $i_{\text {as } 2}$, $i_{a s 3}$ ' and $i_{a s 4}$, which are shown in Fig. 5-2(b). The waveforms of $i_{a s 2}$ ' and $i_{a s 4}$ ' are caused by zigzag connections. The line current $i_{A}=i_{a s 1}$ ' $+i_{a s 2}{ }^{\prime}+i_{a s 3}$ ' $+i_{a s 4}$ '. From Fig. 5-2(e), it can be seen that the $5^{\text {th }}, 7^{\text {th }}, 11^{\text {th }}, 13^{\text {th }}, 17^{\text {th }}$ and $19^{\text {th }}$ order harmonics are zero in $i_{A}$; thus the lowest order harmonic becomes the $23^{\text {rd }}$. As shown in Fig. 5-2(a), the waveform of $i_{A}$ is almost a sinusoid with 5.09\% THD. In Fig. 5-2(d), the pulsated dc load voltage $v_{L}$ has a higher ripple frequency and a lower ripple amplitude, compared to that of the 18-pulse rectifier; therefore, a ripple free dc current can be obtained easier from the 24-pulse rectifier. 


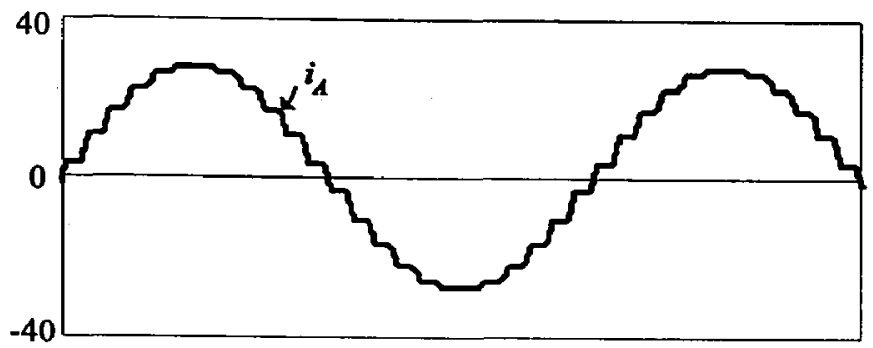

(a) Transformer primary side current $i_{A}$

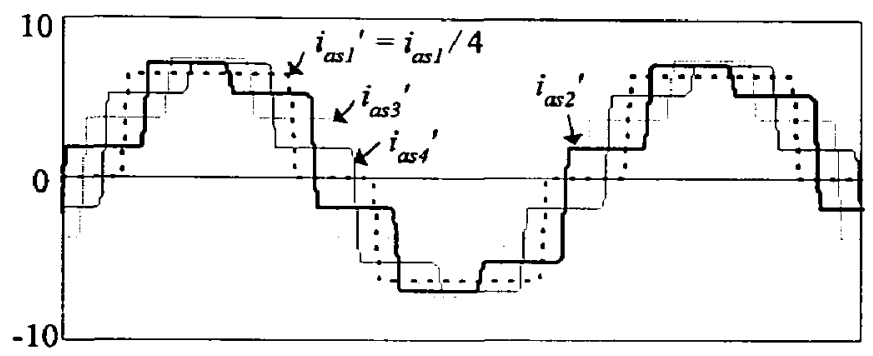

(b) Transformer primary side currents $i_{a s i} i^{\prime} i_{a s 2}{ }^{\prime}, i_{a s 3}, i_{a s 4}^{\prime}$

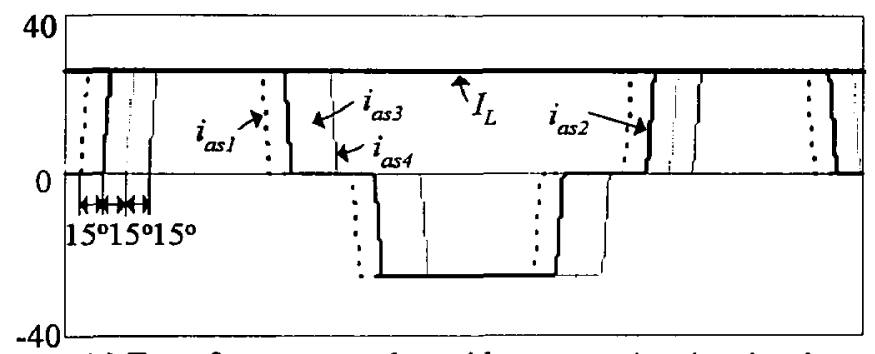

(c) Transformer secondary side currents $i_{a s s}, i_{a s 2}, i_{a s 3}, i_{a s 4}$ and load current $I_{L}$

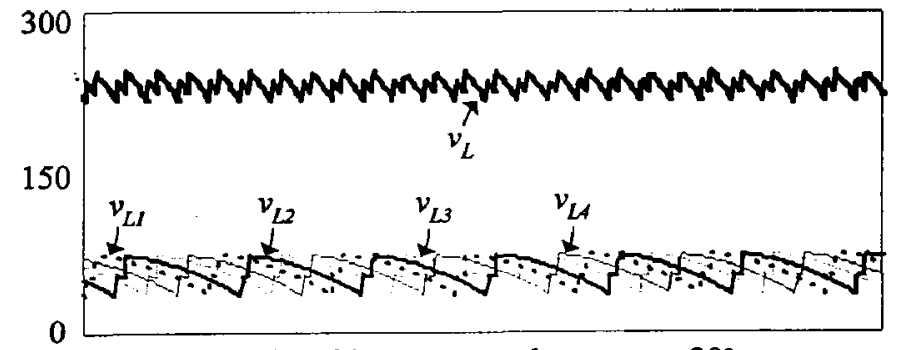

(d) Bridge output voltages at $\alpha=30^{\circ}$

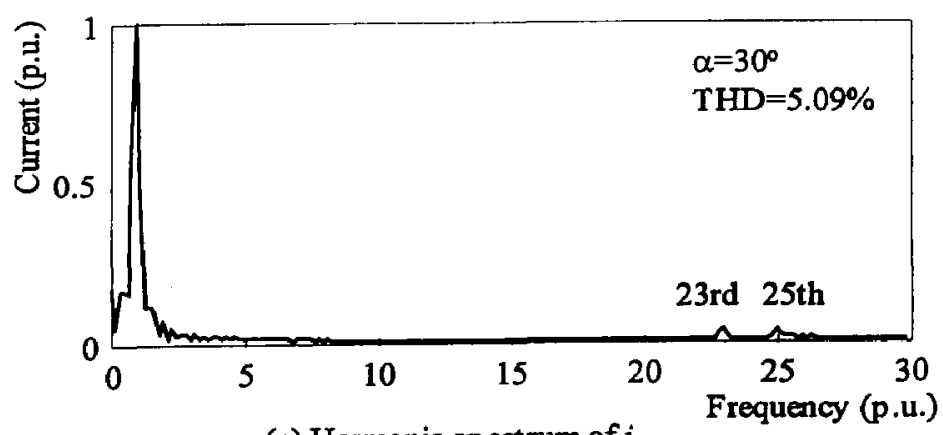

(e) Harmonic spectrum of $i_{A}$

Fig. 5-2 Waveforms for the 24-pulse SCR rectifier with inductive loads. 


\subsubsection{Analysis of Harmonic Elimination}

Fig. 5-3 summarizes the phasor relation of the reflected harmonics being cancelled in the primary winding of the 12-pulse SCR rectifier. Since it's quite straightforward, the details will not be discussed.

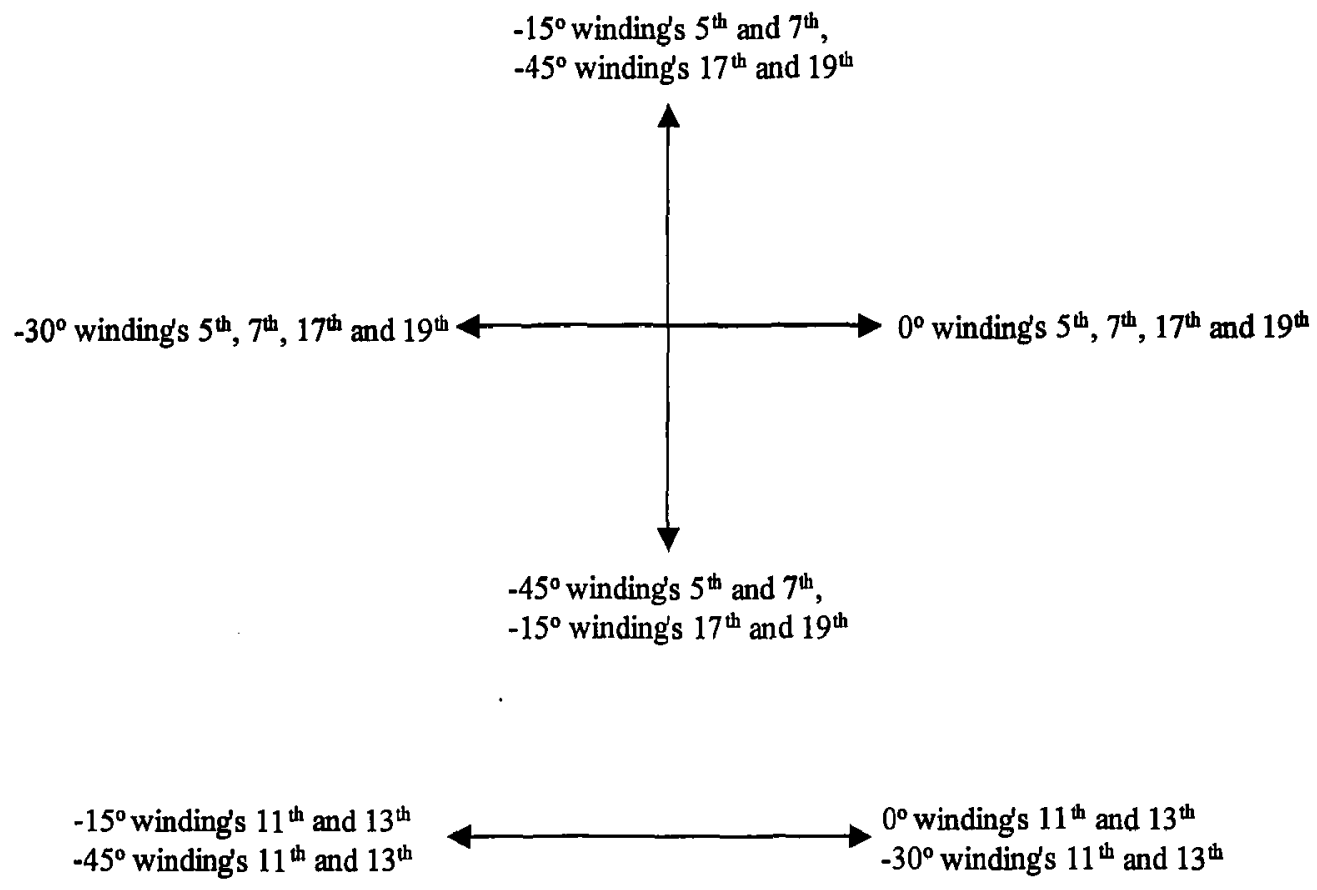

Fig. 5-3 Phasor relation of the reflected harmonics being cancelled in the primary winding of the 24-pulse SCR rectifier.

\subsubsection{The Line Current THD and the Input PF}

Fig. 5-4 shows the line current THD and the input PF of the 24-pulse SCR rectifier with inductive loads under different operating conditions. It $\operatorname{can}$ be seen that when $\alpha=0^{\circ}$ and $I_{A I}>0.2 \mathrm{pu}$, the THD values are less than 5\%, which meet the IEEE Standard 519. 1992. When $\alpha>0^{\circ}$, the THD values are less than $7.5 \%$. The input $\mathrm{PF}$ is almost the same as that of the 18-pulse rectifier. 

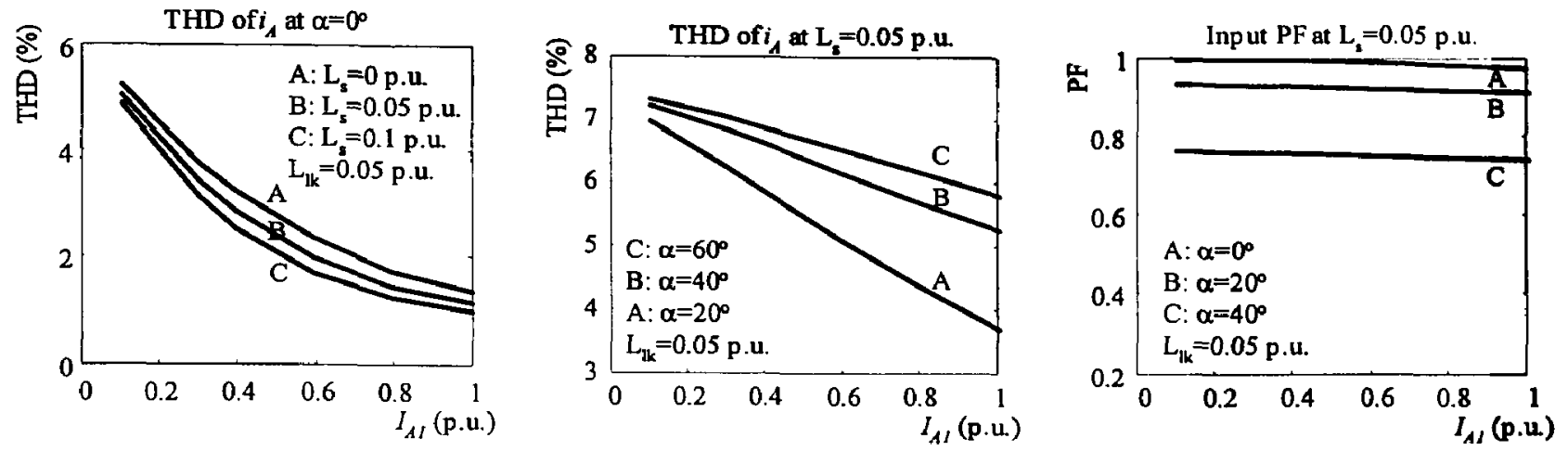

Fig. 5-4 THD and PF curves of the 24-pulse SCR rectifier with inductive loads.

\subsection{4-Pulse SCR Rectifier with Capacitive Loads}

\subsubsection{Circuit Configuration}

Fig. 5-5 shows the simplified diagram of the 24-pulse SCR rectifier with a capacitive load, which is simplified as a dc voltage source $E$.

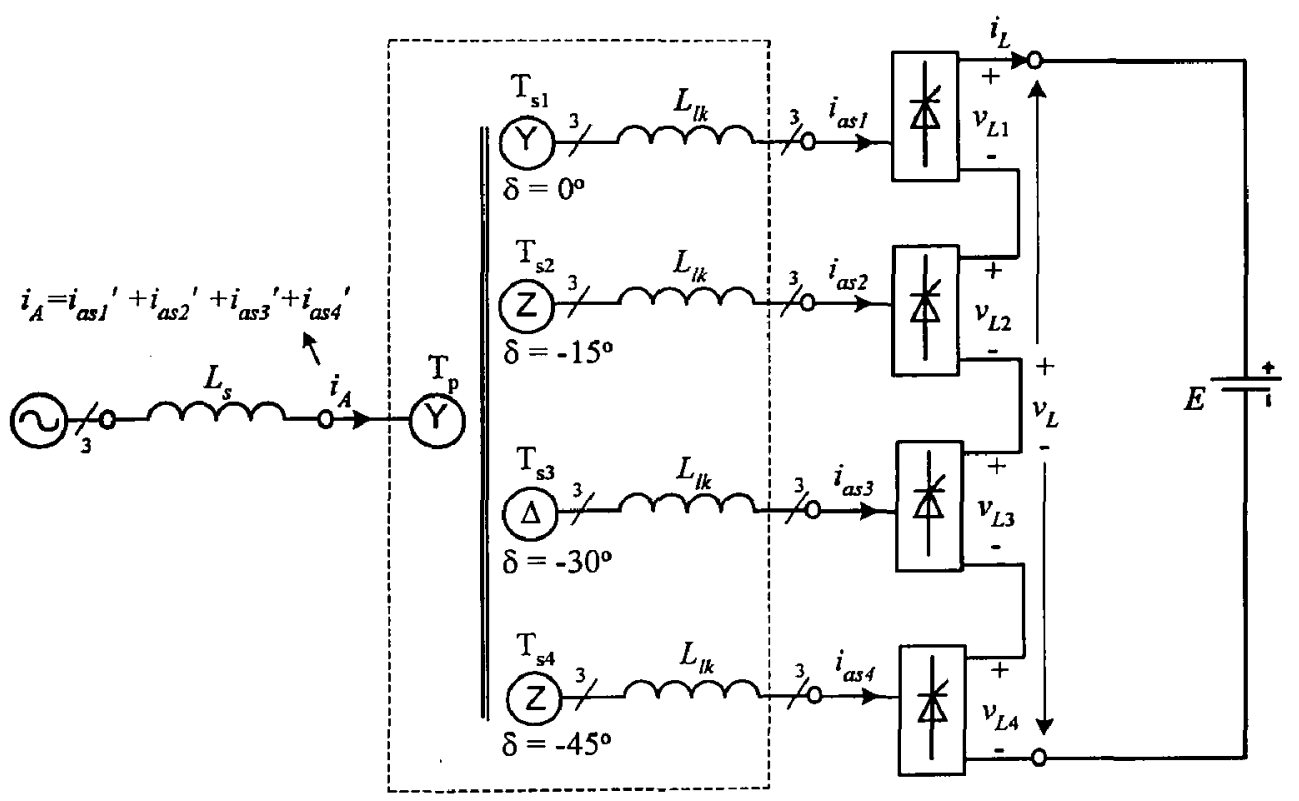

Fig. 5-5 24-pulse SCR rectifier with a capacitive load.

\subsubsection{Current Waveforms}

Fig. 5-6 shows the current waveforms under the condition of $\alpha=30^{\circ}, I_{A I}=1 \mathrm{pu}, L_{s}=0$ and $L_{l k}=0.05 \mathrm{pu}$. The line current $i_{A}$ in Fig. 5-6(a) is almost a sinusoid with little ripples. Fig. 5-6(b) shows the waveforms of $i_{a s I}{ }^{\prime}, i_{a s 2}{ }^{\prime}, i_{a s 3^{\prime}}$ and $i_{a s 4}$ ', in which $i_{a s 2}$ ' and $i_{a s 4}$ ' are caused by zigzag connections. In Fig. 5-6(c), it can be seen that the ripple amount in the 
positive half cycle of each secondary line current is increased to eight and the ripple amplitude is further decreased. Hence a ripple free dc load current can be obtained easier. The harmonic spectrum of $i_{A}$ is shown in Fig. 5-6(e), in which the lowest order harmonic is the $23^{\text {rd }}$, and the THD is $5.2 \%$.

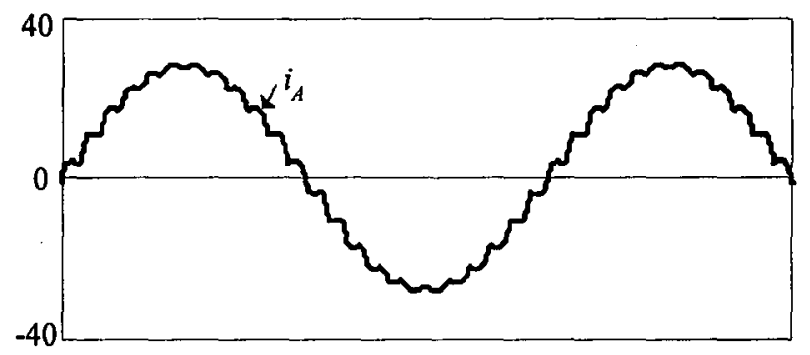

(a) Transformer primary side current $i_{A}$

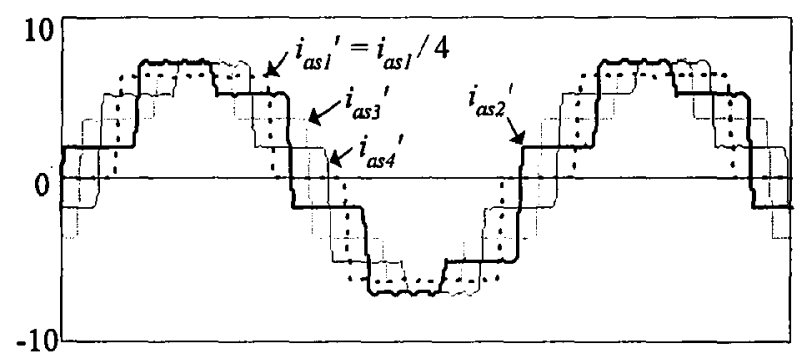

(b) Transformer primary side currents $i_{a s 1}, i_{a s 2}^{\prime}, i_{a s 3}, i_{a s 4}^{\prime}$

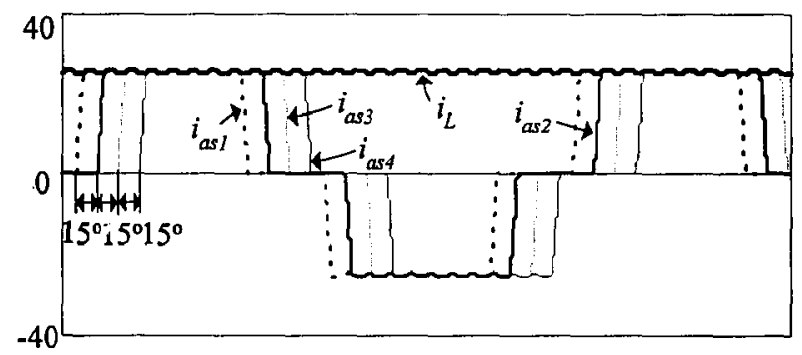

(c) Transformer secondary side currents $i_{a s 1}, i_{a s 2}, i_{a s 3}, i_{a s 4}$ and load current $i_{L}$

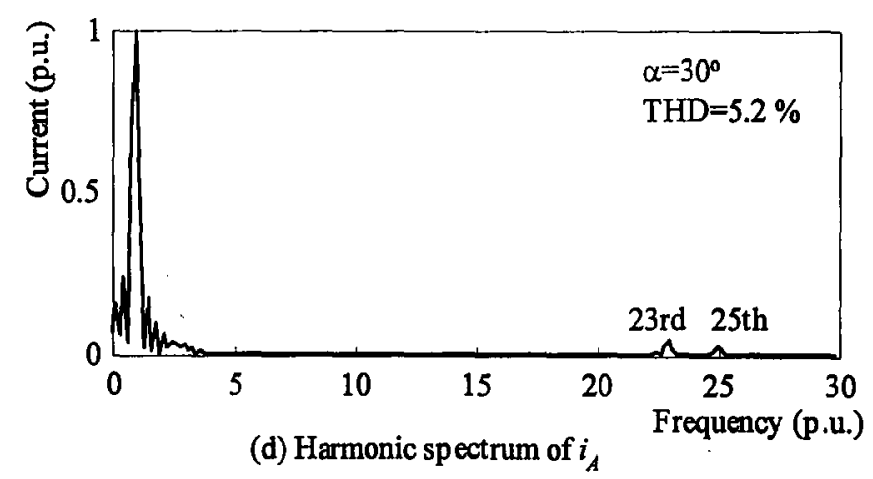

Fig. 5-6 Wạveforms of the 24-pulse SCR rectifier with capacitive loads. 


\subsubsection{The Line Current THD and the Input PF}

Fig. 5-7 shows the waveform and harmonic spectrum of $I_{A I}=1 \mathrm{pu}$ (rated load condition) and $I_{A I}=0.2$ pu (light load condition) at $\alpha=20^{\circ}$. It can be seen that the ripple amplitude of $i_{A}$ is further reduced compared to the 18 -pulse rectifier. Since the $5^{\text {th }}, 7^{\text {th }}$, $11^{\text {th }}, 13^{\text {th }}, 17^{\text {th }}$ and $19^{\text {th }}$ order harmonics are eliminated, the THD of $i_{A}$ is $3.62 \%$ and $7.55 \%$ at $I_{A I}=1 \mathrm{pu}$ and $I_{A I}=0.2 \mathrm{pu}$, respectively, which are lower than those of the 18pulse rectifier.

Fig. 5-8 shows the line current THD and the input PF of the 24-pulse SCR rectifier with capacitive loads under different operating conditions. It can be seen that the THD values are further reduced. Especially when $\alpha=0^{\circ}$, the THD values satisfy the IEEE Standard 519-1992. Under heavy load conditions, the PF of the 24-pulse rectifier is slightly reduced compared to that of the 12- or 18-pulse rectifiers, since the phase displacement of the line current caused by the line inductance and the transformer leakage inductance is slightly greater.
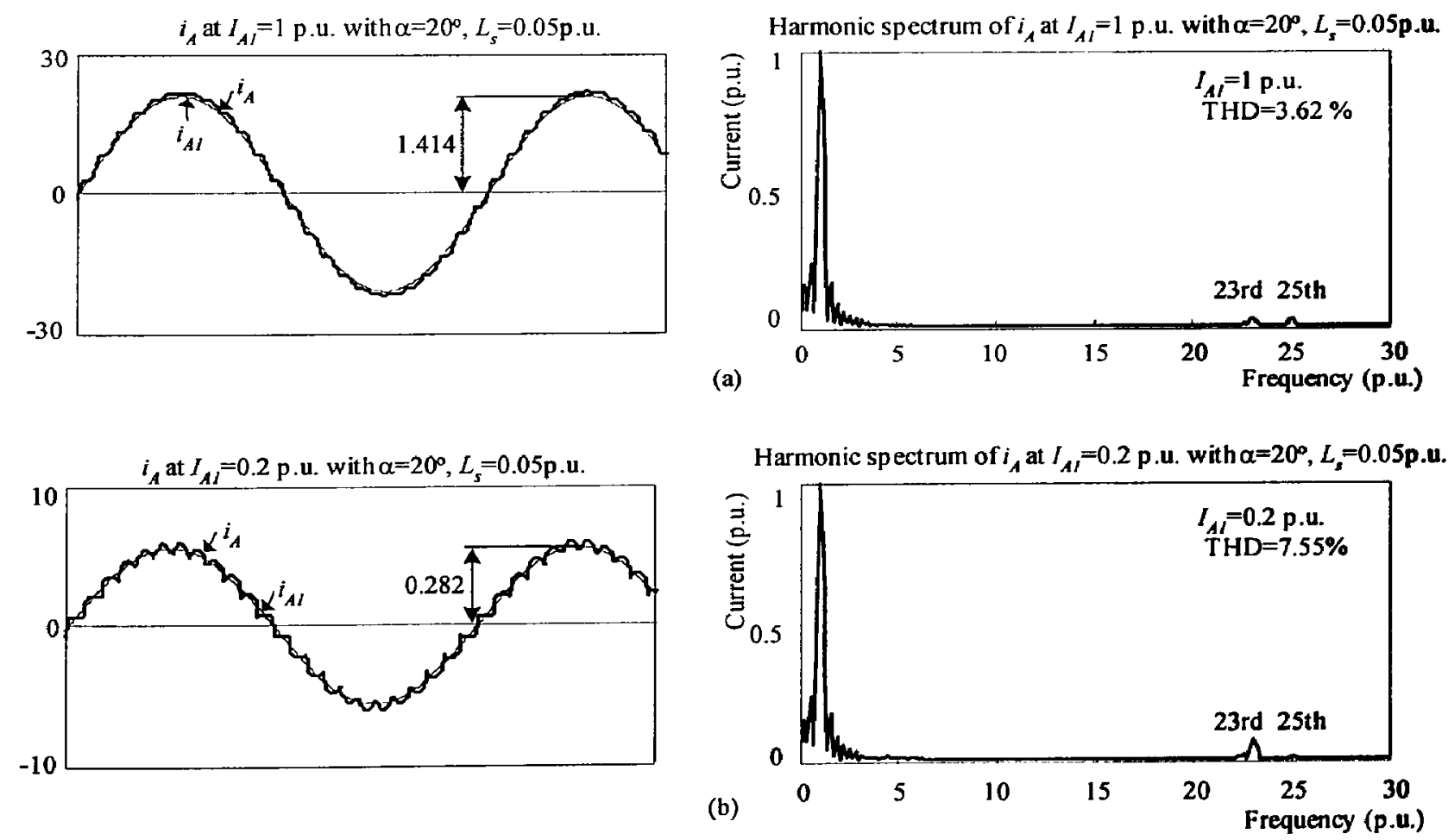

Fig. 5-7 Waveform and harmonic spectrum of $I_{A I}=1 \mathrm{pu}$ and $I_{A I}=0.2 \mathrm{pu}$. 

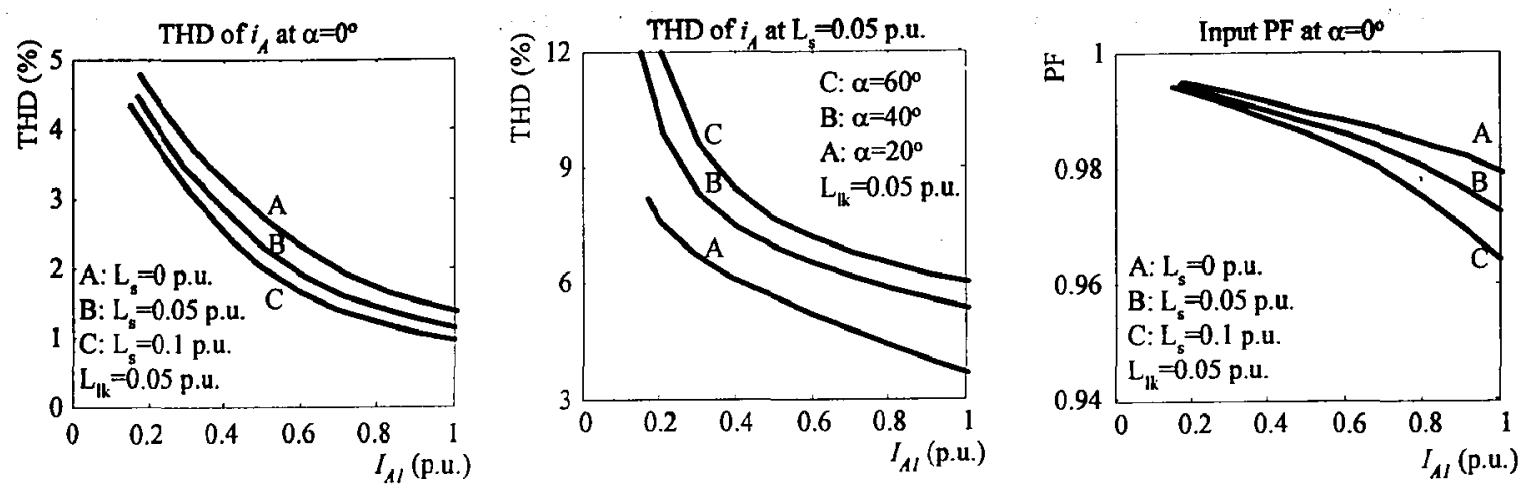

Fig. 5-8 THD and PF curves of the 24-pulse SCR rectifier with capacitive loads.

\subsection{Summary}

This chapter presents the performance of the 24-pulse SCR rectifier with inductive and capacitive loads. The following conclusions can be made:

- The principle of the harmonic elimination can be applied to the 24-pulse rectifier.

- For the two load conditions, since more low-order harmonics in the line current are eliminated, the line current THD of the 24-pulse rectifier is further reduced. Especially when the delay angle $\alpha=0^{\circ}$, the THD values meet the harmonic current requirement of IEEE Standard 519-1992.

- For the capacitive load under heavy load conditions, the PF of the 24-pulse rectifier is slightly reduced, because the phase displacement of the line current caused by the line inductance and the transformer leakage inductance is slightly greater than that of the 12- or 18-pulse rectifier.

- Multi-pulse SCR rectifiers with a higher pulse number, such as the 30- or 36-pulse, are rarely used in industry because of the high cost. 


\section{Chapter 6 Experimental Verification of}

\section{the 12-Pulse SCR Rectifier}

In previous chapters, the performance of multi-pulse SCR rectifiers was presented and the principle of the harmonic elimination through the phase-shifting transformer was discussed. This chapter presents the experimental verification on a prototype of the 12pulse SCR rectifier with an inductive load. The related hardware design and software programming are provided. Typical voltage and current waveforms obtained from the experiment are compared to the simulation results.

\subsection{Hardware Configuration of the Experimental System}

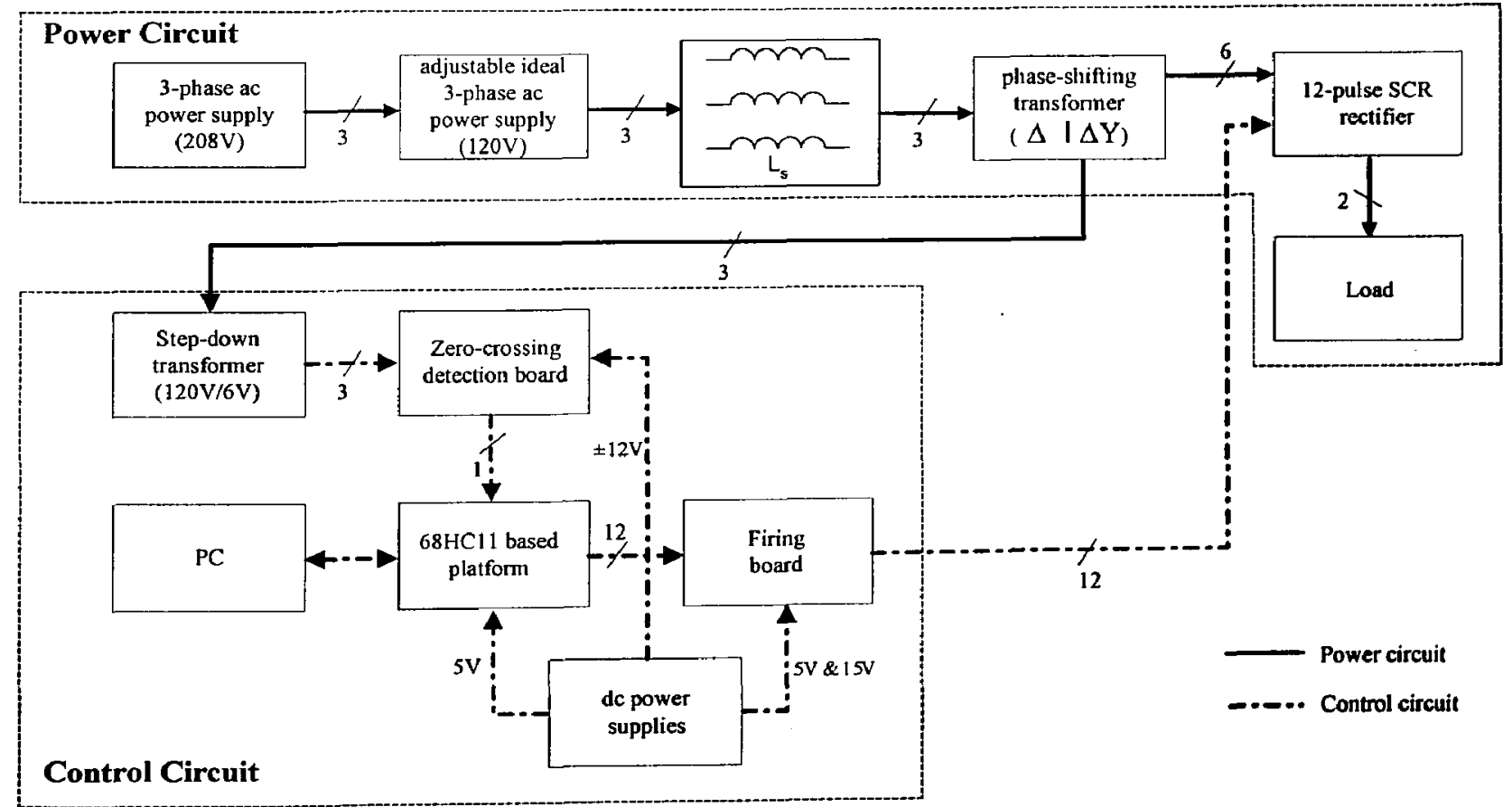

Fig. 6-1 Hardware configuration of the experimental system. 
Fig. 6-1 shows the hardware configuration of the experimental 12-pulse SCR rectifier, which consists of the power circuit and the control circuit.

\section{Power Circuit}

The power circuit of the 12-pulse SCR rectifier includes a three-phase sinusoidal ac power supply, an inductor bank $L_{s}$, a phase-shifting transformer $(\Delta \mid \Delta \mathrm{Y})$, two six-pulse SCR bridges, and a load.

Three-phase sinusoidal ac power supply: SW5250A is a three-phase ac power supply, which generates adjustable sinusoidal voltages. The maximum current of the device is $13 \mathrm{~A}$ (RMS) with the output voltage range of $0-156 \mathrm{~V}$ (RMS) [11].

The inductor bank $L_{s}$ : Since the internal impedance of the three-phase sinusoidal power supply is low, the inductor $L_{s}$ is needed for emulating the actual occasions.

Phase-shifting transformer: As shown in Fig. 6-2, the phase-shifting transformer of the 12-pulse rectifier has two secondary windings, which are $\Delta$ and $Y$ connected, feeding two six-pulse SCR bridges, respectively. From the phase-shifting transformer nameplate, the following parameters are obtained:

total rated apparent power for the transformer primary winding: $20 \mathrm{KVA}$;

rated apparent power for each transformer secondary winding: $10 \mathrm{KVA}$;

rated RMS line-to-line voltage of the transformer primary winding: $208 \mathrm{~V}$; rated RMS line-to-line voltage of each transformer secondary winding: $208 \mathrm{~V}$; total rated line current of the transformer primary winding: $55.5 \mathrm{~A}$; rated line current of each transformer secondary winding: $27.7 A$; $\%$ impedance between the transformer primary winding and secondary winding base on $20 K V A: 4.1 \%$.

SCR rectifier: The 12-pulse SCR rectifier has two six-pulse SCR bridges, whose dc outputs are connected in series. 
Load: The load of the 12-pulse rectifier is a resistor bank connected in series with a $40 m H$ inductor bank.

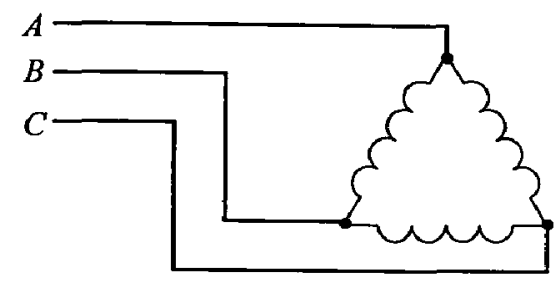

Fig. 6-2

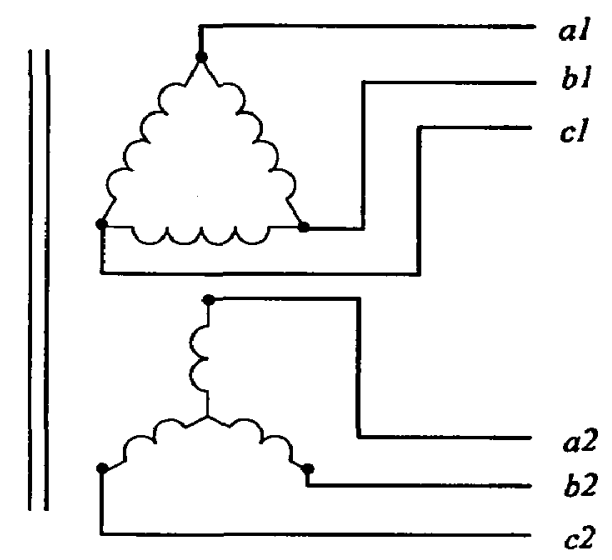

Configuration of the phase-shifting transformer.

\section{Control Circuit}

The control circuit consists of a step-down transformer (120V/6V), a zero-crossing detection board, a MC68HC11 control board, a SCR firing board, dc power supplies and a personal computer.

Step-down transformer (120V/6V): The step-down transformer transfers the input phase voltages from $120 V(\mathrm{RMS})$ to $6 V(\mathrm{RMS})$, so as to meet the requirements of the zerocrossing detection board.

MC68HC11 control board: The SCR gating pulses of the 12-pulse rectifier are generated by the MC68HC11 control board.

Firing board: The gating pulses generated by the MC68HC11 control board are amplified and isolated by the firing board to meet the triggering requirement of SCRs.

Dc power supplies: the dc power supplies provide $5 \mathrm{~V}, \pm 12 \mathrm{~V}$ and $15 \mathrm{~V}$ dc voltages to the control circuit. 
Personal computer: the personal computer instructs the MC68HC11 control board to realize the real-time control.

\subsection{Parameter Design of the Experimental System}

Considering the power limit of the three-phase ac power supply SW5250A, $2.2 \mathrm{KVA}$ apparent power and $120 \mathrm{~V}$ RMS line-to-line voltage are selected. The related calculations are given as follows:

\section{Rectifier Calculation (assuming $L_{s}=0$ and $L_{l k}=0$ )}

Since the rated supply RMS line-to-line voltage $V_{L L}=120 \mathrm{~V}$, from Equation (2.7), the average output voltage of each six-pulse SCR rectifier under ideal circuit condition is $V_{L 1(2), \text { avg }}=1.35 V_{L L} \cos \alpha=1.35 \times 120 \times \cos \alpha=162 \cos \alpha$, where $\alpha$ is the delay angle. From Equation (3.8), the average output voltage of the 12-pulse rectifier with an inductive load is $V_{L, \text { avg }}=1.93 \times 162 \cos \alpha=313 \cos \alpha$ and the average load current is $I_{L, \text { avg }}=V_{L, \text { avg }} / R_{L}=313 \cos \alpha / R_{L}$. When $R_{L}=40 \Omega$ and $\alpha=30^{\circ}, V_{L, \text { avg }}=313 \cos 30^{\circ}=271 \mathrm{~V}$ and $I_{L, a v g}=271 / 40=6.78 \mathrm{~A}$. Considering the transformer turns ratio, we get $I_{A, R M S}=2 \times 0.7884 I_{L}=2 \times 0.7884 \times 6.78=10.7 \mathrm{~A}$.

\section{Per Unit Calculation}

It's convenient to use per unit system to analyze the power systems. For the phaseshifting transformer, the base transformer apparent power $S_{R, T}=20 K V A$ and the base line-to-line voltage $V_{L L, T}=208 \mathrm{~V}(\mathrm{RMS})$, then:

the base voltage $V_{B, T}=\frac{V_{L L, T}}{\sqrt{3}}=\frac{208}{\sqrt{3}}=120 \mathrm{~V}$;

the base current $I_{B, T}=\frac{S_{R, T}}{3 V_{B, T}}=\frac{20000}{3 \times 120}=55.56 \mathrm{~A}$;

the base impedance $Z_{B, T}=\frac{V_{B, T}}{I_{B, T}}=\frac{120}{55.56}=2.16 \Omega$;

the base angular frequency $\omega_{B, T}=2 \pi f=2 \pi \times 60=377 \mathrm{rad} / \mathrm{s}$; 
the base inductance $L_{B, T}=\frac{Z_{B, T}}{\omega_{B, T}}=\frac{2.16}{377}=5.73 \mathrm{mH}$;

the base capacitance $C_{B, T}=\frac{1}{\omega_{B, T} Z_{B, T}}=\frac{1}{377 \times 2.16}=1.228 \mathrm{mF}$.

Therefore, $\% 4.1 Z_{B T}=2.16 \times 4.1 \%=0.08856 \Omega$, and the real value of the total transformer leakage inductance $L_{l k}=\frac{0.08856}{377}=0.235 \mathrm{mH}$, or $L_{l k}=4.1 \% \times 5.73 \mathrm{mH}$ $=0.235 \mathrm{mH}$

For the experimental system, the base transformer apparent power $S_{R}=2.2 \mathrm{KVA}$ and the base line-to-line voltage $V_{L L}=120 \mathrm{~V}(\mathrm{RMS})$, then:

the base voltage $V_{B}=\frac{V_{L L}}{\sqrt{3}}=\frac{120}{\sqrt{3}}=69.28 \mathrm{~V}$;

the base current $I_{B}=\frac{S_{R}}{3 V_{B}}=\frac{2200}{3 \times 69.28}=10.585 \mathrm{~A}$;

the base impedance $Z_{B}=\frac{V_{B}}{I_{B}}=\frac{69.28}{10.585}=6.545 \Omega$;

the base angular frequency $\omega_{B}=2 \pi f=2 \pi \times 60=377 \mathrm{rad} / \mathrm{s}$;

the base inductance $L_{B}=\frac{Z_{B}}{\omega_{B}}=\frac{6.545}{377}=17.36 \mathrm{mH}$;

the base capacitance $C_{B}=\frac{1}{\omega_{B} Z_{B}}=\frac{1}{377 \times 6.545}=405 \mu \mathrm{F}$;

the total transformer leakage inductance $L_{l k}=\frac{0.235 \mathrm{mH}}{17.36 \mathrm{mH}}=0.0135 \mathrm{pu}$.

\section{$L_{s}$ Calculation}

A three-phase inductor bank is added to the circuit to emulate the actual occasions. Choose $L_{s}=1 m H$; then based on the experimental system, $L_{s}=1 m H=\frac{1 m H}{17.36 m H}$ $=0.0576 \mathrm{pu}$, which is a reasonable value. 


\section{$i_{A}$ THD Calculation}

From Equation (2.18), $T H D_{i A}=\frac{\sqrt{I_{A, R M S}^{2}-I_{A 1, R M S}^{2}}}{I_{A 1, R M S}}$, the $i_{A}$ THD of the experimental setup can be calculated based on $I_{A, R M S_{-} \text {exp, }}$, the RMS value of the line current, and

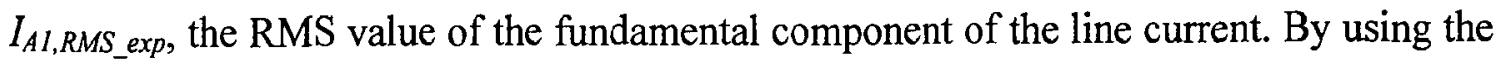
measurement function of the oscilloscope, we have $I_{A, R M S \text { exp }}$. Through the FFT function, we can get the harmonic spectrum of the line current, of which the measured peak value is $I_{A l, R M S_{-} \text {exp }}$. Thus the $i_{A}$ THD of the experimental system can be obtained.

\subsection{Software Programming and Hardware Implementation of the Experimental System}

\subsubsection{Software Programming}

In order to generate SCR gating signals for the 12-pulse rectifier, the MC68HC11 programs are designed. The software development is based on the MiniDE for personal computers.

\subsubsection{Hardware Implementation}

After the hardware connection and software programming, the whole experimental setup is ready for the following procedures:

Step 1: Check the three-phase power supply. Use the oscilloscope to make sure that the amplitude and sequence of the three phase voltages are correct.

Step 2: Set the SW5250A to the designed voltage and current values, use the oscilloscope to check the voltage sequence, and output the three phase voltages to the phaseshifting transformer primary winding.

Step 3: Use the oscilloscope to measure the amplitude and the sequence of the output line-to-line voltages of the two transformer secondary windings, and feed them to the two six-pulse SCR bridges, respectively.

Step 4: Set the resistor bank to the desired values.

Step 5: Run the control circuit and output gating pulses to the 12-pulse SCR rectifier.

Step 6: Tune the delay angle $\alpha$ to get the desired voltage and current waveforms, and record the results. 


\subsection{Experimental Results}

\section{Source Voltages}

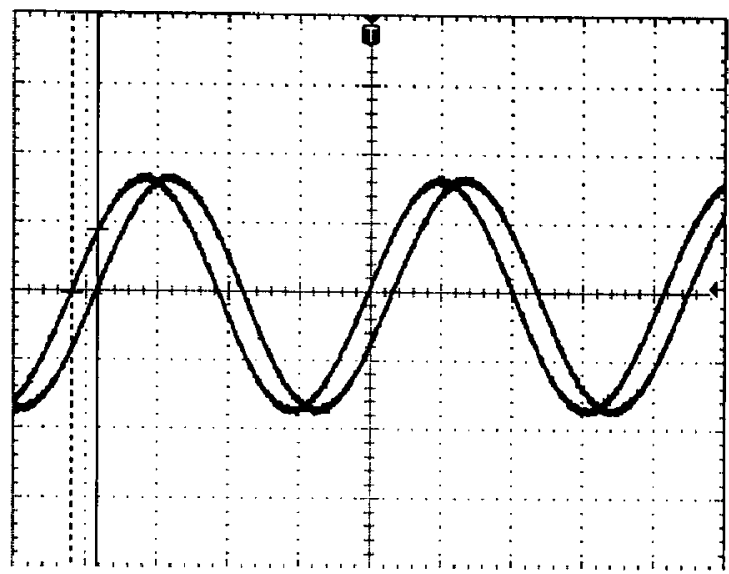

Ch1 RMS $118 \mathrm{~V}$ Ch2 RMS $116 \mathrm{~V}$

Fig. 6-3 Measured source voltage waveforms.

Fig. 6-3 shows the line-to-line voltage waveforms of the two transformer secondary windings, in which a $30^{\circ}$ phase-shift can be observed. The measured RMS line-to-line voltages of the two windings are $118 \mathrm{~V}$ and $116 \mathrm{~V}$, respectively.

\section{Zero-Crossing Signal}

In Fig. 6-4, the sinusoidal waveform is the line-to-line voltage $v_{a c}$ of the wyeconnected secondary winding, and the square waveform is the output signal $v_{Z C}$ of the zero-crossing detection board. $v_{a c}$ is the phase $A$ zero-crossing reference signal, whose zero-crossing points are corresponding to the nature commutation points of phase $A$ in a three-phase system. Hence the delay angle $\alpha$ could be measured with respect to the $v_{a c}$ zero crossing points, which are corresponding to the rising and falling edges of $v_{Z C}$. 


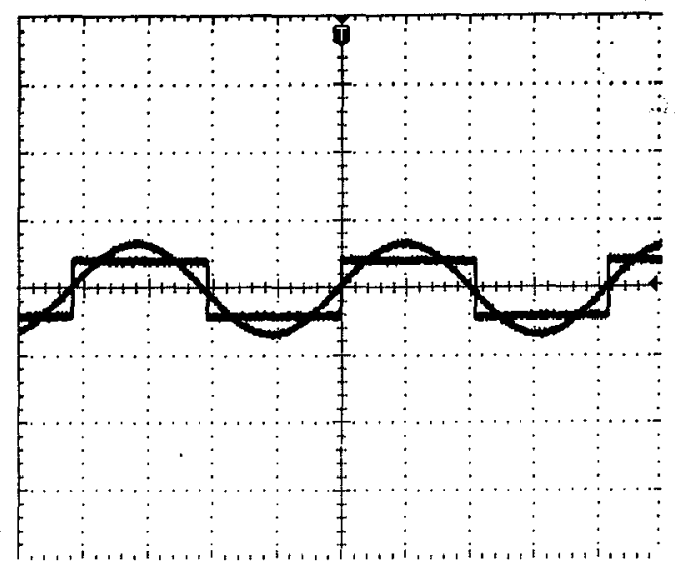

Fig. 6-4 Measured $v_{a c}$ and $v_{z c}$.

\section{Gating Pulses Generated by the MC68HC11 Control Board}

Fig. 6-5 shows the twelve gating pulses generated by the MC68HC11 control board. The condition of the plot is $\alpha=30^{\circ}$ and pulse width $=120^{\circ}$. The applicable range of $\alpha$ is from $1^{\circ}$ to $179^{\circ}$.

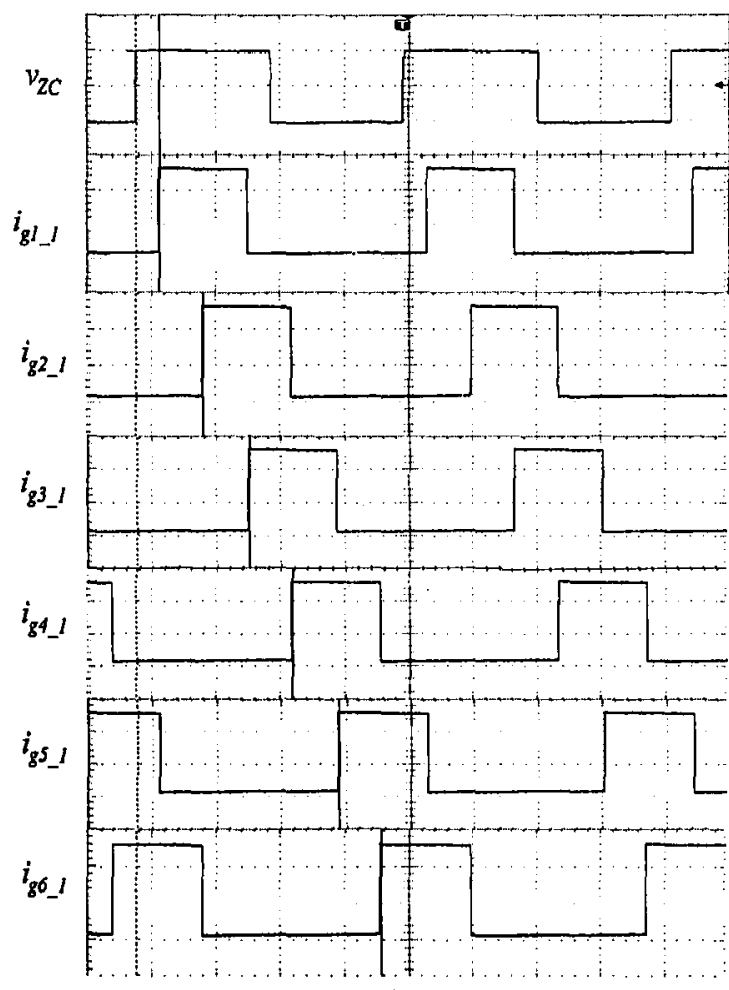

(a)

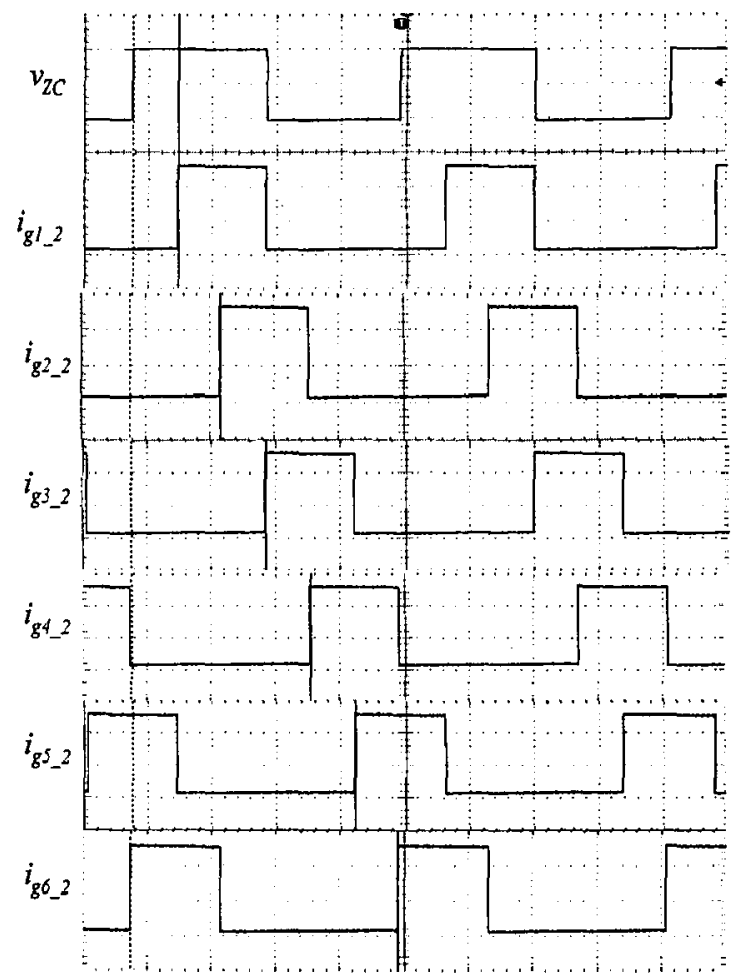

(b)

Fig. 6-5 Gating pulses generated by the $\mathrm{MC} 68 \mathrm{HC} 11$ control board.

(a) Gating pulses for the $1^{\text {st }}$ six-pulse SCR bridge.

(b) Gating pulses for the $2^{\text {nd }}$ six-pulse SCR bridge. 
It can be seen that the two sets of gating pulses are phase-shifted by $30^{\circ}$, which meets the gating requirement of the 12-pulse SCR rectifier. For the first gating pulse $i_{g I_{-}}$, the delay angle $\alpha$ is measured right after the rising edge of $v_{Z C}$. The phase displacement between any adjacent gating pulses in each set is $60^{\circ}$.

\section{2-Pulse SCR Rectifier Voltage and Current Waveforms}

Fig. 6-6 shows the measured voltage and current waveforms of the 12-pulse SCR rectifier with the inductive load at $\alpha=30^{\circ}$. The simulation results are provided for comparison. Fig. 6-6(a) and Fig. 6-6(b) show the measured line current waveforms of the two transformer secondary windings, respectively. A $30^{\circ}$ phase shift can be identified when they are shown on the oscilloscope simultaneously. From the harmonic spectrum, it can be seen that the $5^{\text {th }}, 7^{\text {th }}, 17^{\text {th }}$ and $19^{\text {th }}$ order harmonics exist in the two currents and produce a THD of $34.4 \%$.

Fig. 6-6(c) shows the measured current waveform of the transformer primary winding, which looks more like a sinusoid compared to Fig. 6-6(a) and Fig. 6-6(b). From the harmonic spectrum, it can be seen that the $5^{\text {th }}, 7^{\text {th }}, 17^{\text {th }}$ and $19^{\text {th }}$ order harmonics are eliminated. Therefore the THD of $i_{A}$ is reduced to $17.52 \%$.

Fig. 6-6(d) shows the measured output voltage waveforms of the two six-pulse SCR bridges. It can be measured that the phase shift between the two voltages is $30^{\circ}$. Fig. 66(e) shows the measured dc load voltage waveform of the 12-pulse SCR rectifier. Compared to those in Fig. 6-6(d), the dc ripple frequency in Fig. 6-6(e) is doubled. Fig. 6$6(\mathrm{f})$ shows the measured dc load current, which has a mean value of $5.41 \mathrm{~A}$.

Since losses and fluctuations exist in the real system, the experimental results in Fig. 6-6 have a lower line current RMS, a higher line current THD, and a lower mean de load current than the simulation results. 


\subsection{Summary}

This chapter presents the experimental verification of the performance of multi-pulse $\mathrm{SCR}$ rectifiers on a prototype of the 12-pulse $\mathrm{SCR}$ rectifier with an inductive load. From the experimental results, we can get the following conclusions:

- The $5^{\text {th }}, 7^{\text {th }}, 17^{\text {th }}$ and $19^{\text {th }}$ order harmonics exist in the transformer secondary winding line currents.

- The $5^{\text {th }}, 7^{\text {th }}, 17^{\text {th }}$ and $19^{\text {th }}$ order harmonics do not exist in the transformer primary winding line currents.

- The line current THD of the 12-pulse SCR rectifier is lower than that of the six-pulse SCR rectifier.

- The dc ripple frequency of the 12-pulse rectifier is doubled, compared to that of the six-pulse rectifier.

- Losses and fluctuations in the experimental circuit slightly influence the experimental results.

- The principle presented in Chapter 3 is verified. 


\section{Experimental Result}

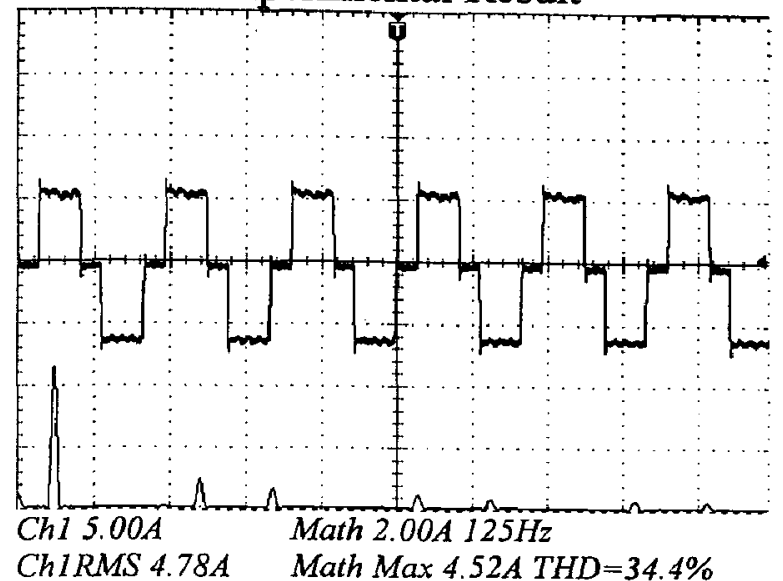

(a) Line current $i_{a s ~}$ of the $1^{\text {st }}$ six-pulse bridge.

(Transformer secondary side line current)

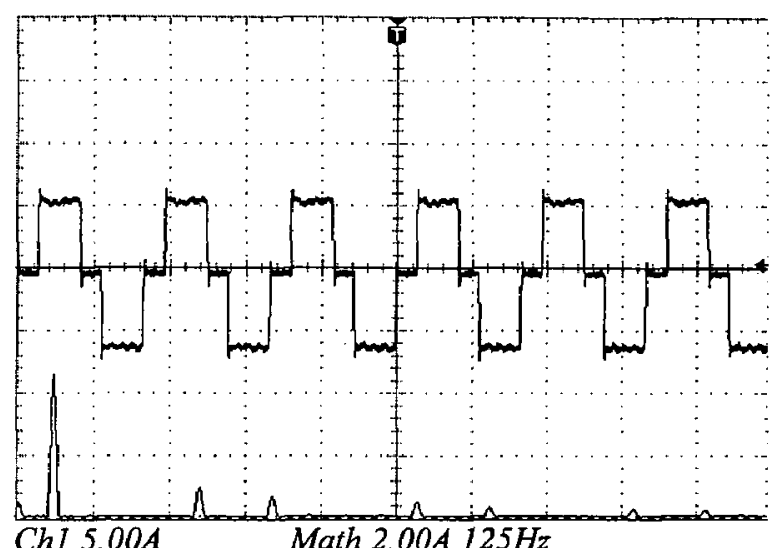

Math 2.00A $125 H z$

ChIRMS 4.78A Math Max 4.52A THD=34.4\%

(b) Line current $i_{a s 2}$ of the $2^{\text {nd }}$ six-pulse bridge.

(Transformer secondary side line current)

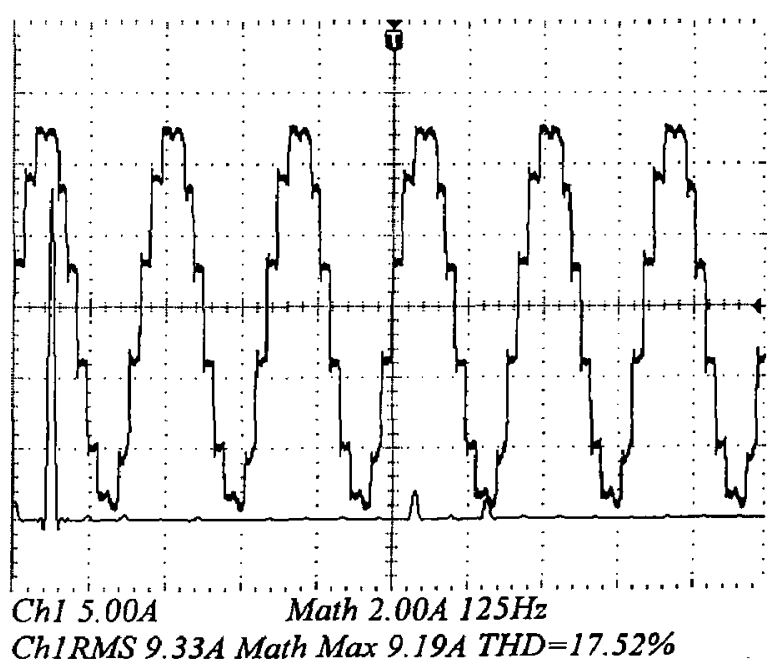

(c) Line current $i_{A}$ of the 12-pulse rectifier.

(Transformer primary side line current)
Simulation Result
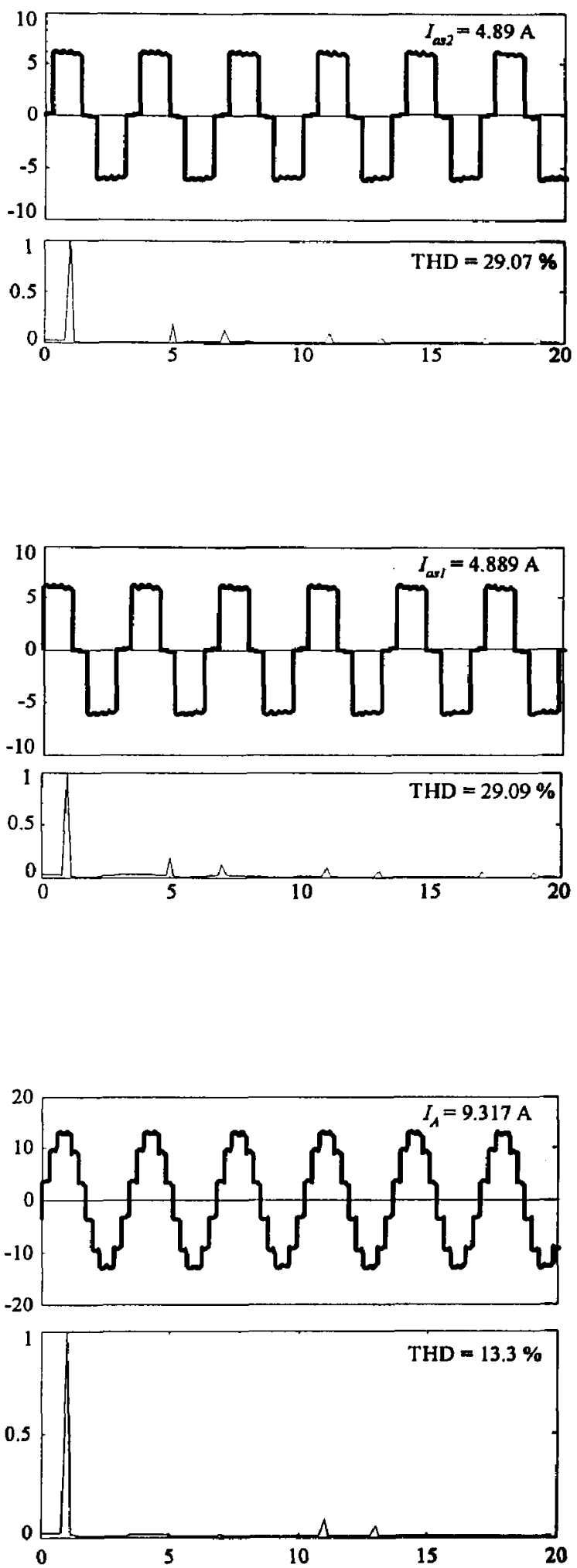


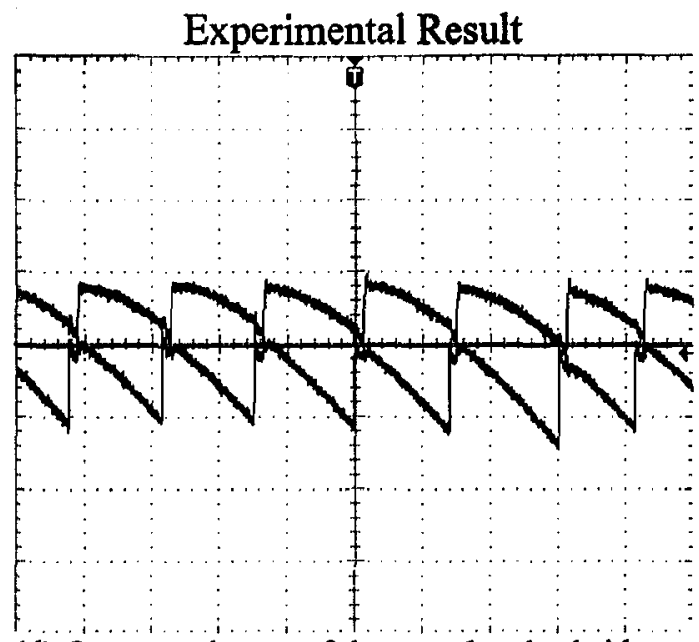

Simulation Result

(d) Output voltages of the two 6-pulse bridges.
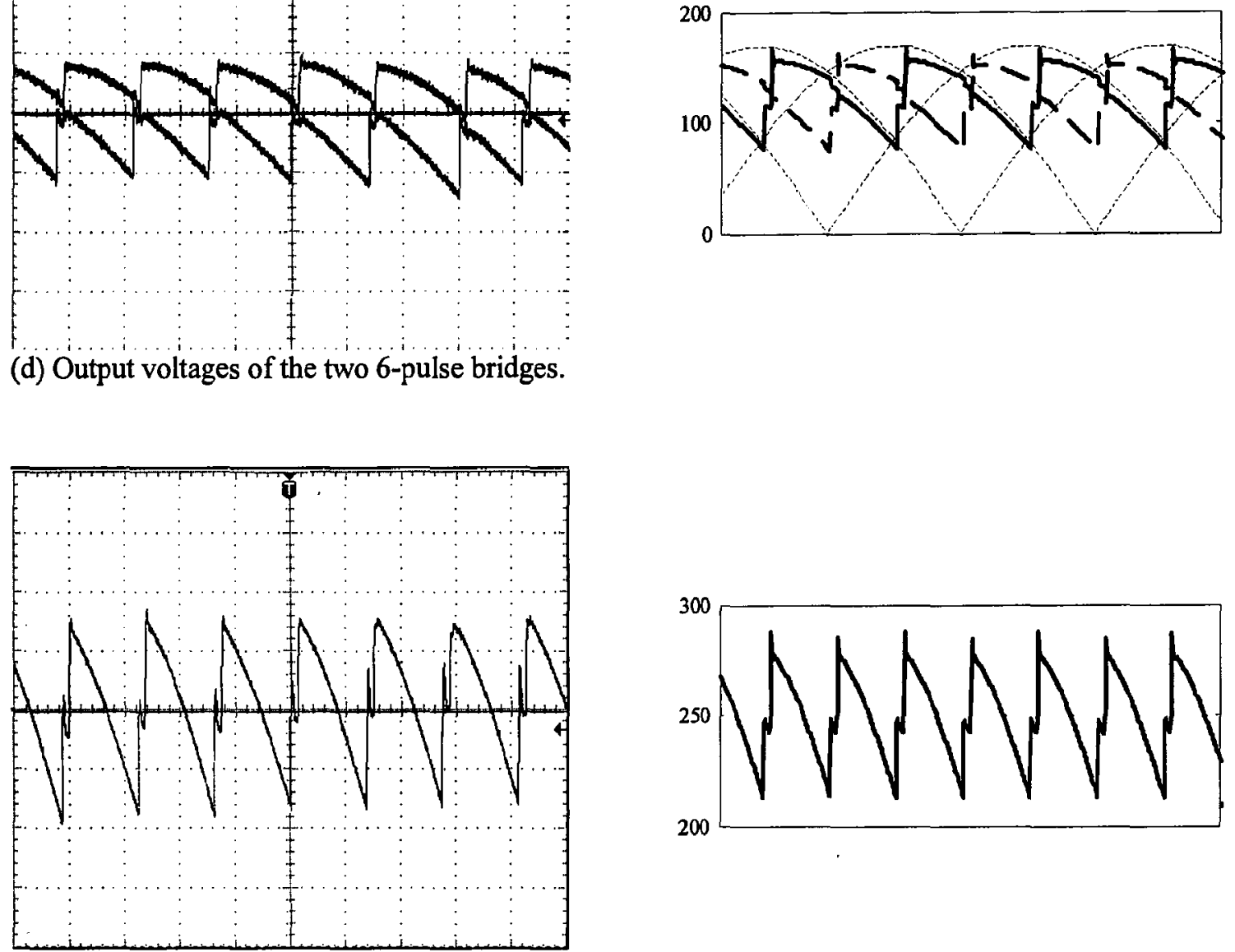

(e) Load voltage of the 12-pulse rectifier.
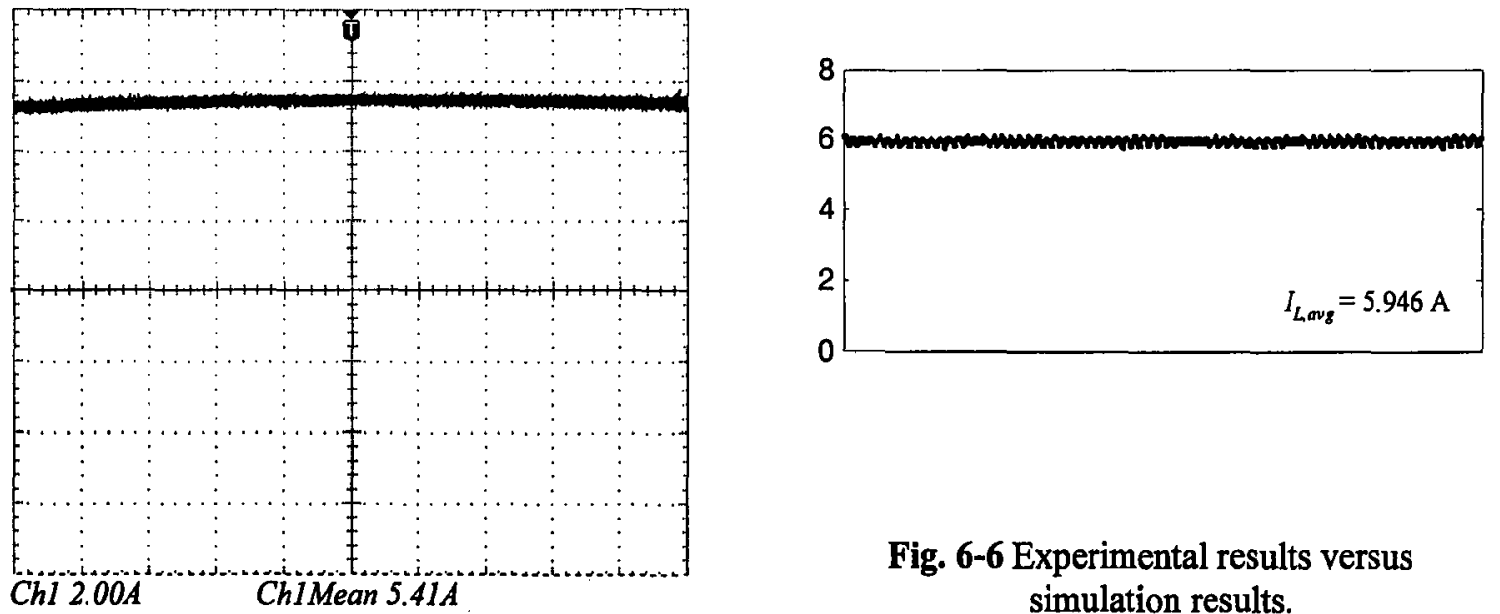

(f) Load current of the 12-pulse rectifier.

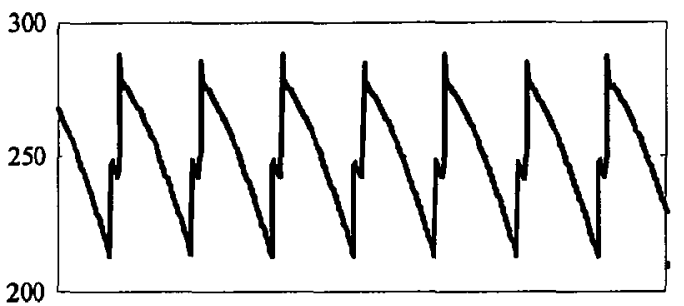

Fig. 6-6 Experimental results versus simulation results. 


\section{Chapter 7 Conclusions}

Multi-pulse SCR rectifiers are widely used in high power applications in industry due to their low line current Total Harmonic Distortion (THD) and high voltage capabilities. The main contribution of the thesis is to analyze the performance of the six-, 12-, 18- and 24-pulse SCR rectifiers with inductive and capacitive loads. The line current THD and the input Power Factor (PF) of the rectifiers are investigated and the results are shown in graphics for engineering references. The principle of the harmonic elimination through the phase-shifting transform is analyzed by Fourier analysis and positive/negative sequence analysis. Experimental verification is completed on a prototype of the 12-pulse SCR rectifier.

As a basic three-phase controllable ac-dc converter, the six-pulse SCR rectifier is seldom applied in high power applications because of its high line current THD, low input PF and voltage sharing problem occurred when SCRs are connected in series. The drawbacks of the six-pulse SCR rectifier could be overcome by using multi-pulse SCR rectifiers, in which six-pulse rectifiers work as the building block. The benefit of using multi-pulse SCR rectifiers includes:

- The line current THD is reduced.

Under the same operating condition, the larger the number of pulses, the more the loworder harmonics can be eliminated, and the lower the line current THD is. For instance, the THD of the 18-pulse SCR rectifier is lower than that of the 12-pulse rectifier.

- The output de ripple frequency in the multi-pulse rectifiers is increased and the ripple amplitude is decreased.

The dc ripple frequency of the 12-, 18- and 24-pulse rectifiers is increased to two, three and four times of that in the six-pulse rectifier, respectively. The peak-to-peak dc ripple amplitude decreases as the pulse number of the rectifier increases. Therefore, it is easier to obtain a ripple free dc output in the rectifier with a larger pulse number. 
- The voltage sharing problem in the in-series SCRs is avoided.

When SCRs are connected in series to handle high voltages, simultaneous operating of the SCRs has to be ensured. In multi-pulse rectifiers, the SCR bridges are connected in series rather than SCRs, and hence the voltage sharing problem of the in-series SCRs is avoided. The larger the number of pulses, the more SCR bridges should be connected in series, and the higher voltage the rectifier can handle.

Using phase-shifting transformers in multi-pulse SCR rectifiers can cancel certain low-order harmonics from the rectifiers' line current. The principle of the harmonic elimination through phase-shifting transformers is analyzed by Fourier analysis and positive/negative sequence analysis. The harmonics that appear in the line current of multi-pulse rectifiers under normal operating conditions are the $(k q \pm 1)^{\text {th }}$, where $q$ is the pulse number of the rectifier and $k=1,2,3 \ldots$

In order to verify the performance of the multi-pulse SCR rectifiers, a prototype of the 12-pulse SCR rectifier controlled by a microprocessor is designed and constructed. The experimental results show that the low-order harmonics, such as the $5^{\text {th }}$ and $7^{\text {th }}$, are eliminated from the line current of the 12-pulse SCR rectifier. As a result, its line current THD is reduced substantially compared to that of the six-pulse rectifier. 


\section{Appendix A MC68HC11 Programming}

; 12 gating pulses generation for rectifier circuits

; width of output pulse 120 degree

; 1 interrupt

; ALPHA (1 179 degree)

; define subroutine addresses located in EPROM

INCHAR EQU \$E55B;subroutine to get strings from keyboard

OUTSTR EQU \$E52F; subroutine to display strings on monitor

WCTRL EQU \$E096 ;subroutine to control LCD configuration

WDAT EQU \$E0A3 ;subroutine to display strings on LCD

;Define addresses of $\mathrm{HC} 11$ registers used in this program

TCNT EQU \$100E ;timer counter register (free running)

TIC1 EQU \$1010;input capture register 1

TIC2 EQU \$1012;input capture register 2

TIC3 EQU \$1014;input capture register 3

TOC1 EQU $\$ 1016$;output compare register 1

TOC2 EQU $\$ 1018$; output compare register 2

TOC3 EQU \$101A ;output compare register 3

TOC4 EQU \$101C ;output compare register 4

TOC5 EQU \$10IE ; output compare register 5

TCTL2 EQU \$1021 ;timer control register 2

TMSK 1 EQU \$1022 ; ;imer interrupt mask register 1

TFLG1 EQU $\$ 1023$;timer interrupt flag register 1

PACTL EQU \$1026 ;pulse accumulator control resister

DDRD EQU $\$ 1009$; data direction for Port D

PORTA EQU $\$ 1000 \quad$;port A

;PORTB EQU $\$ 1100 \quad$;port B chip address, direct write to address.

PORTD EQU $\$ 1008$;port D

;PERIOD EQU 2778 ;

;pulse EQU 1158 ;

ORG $\$ 6000$ 


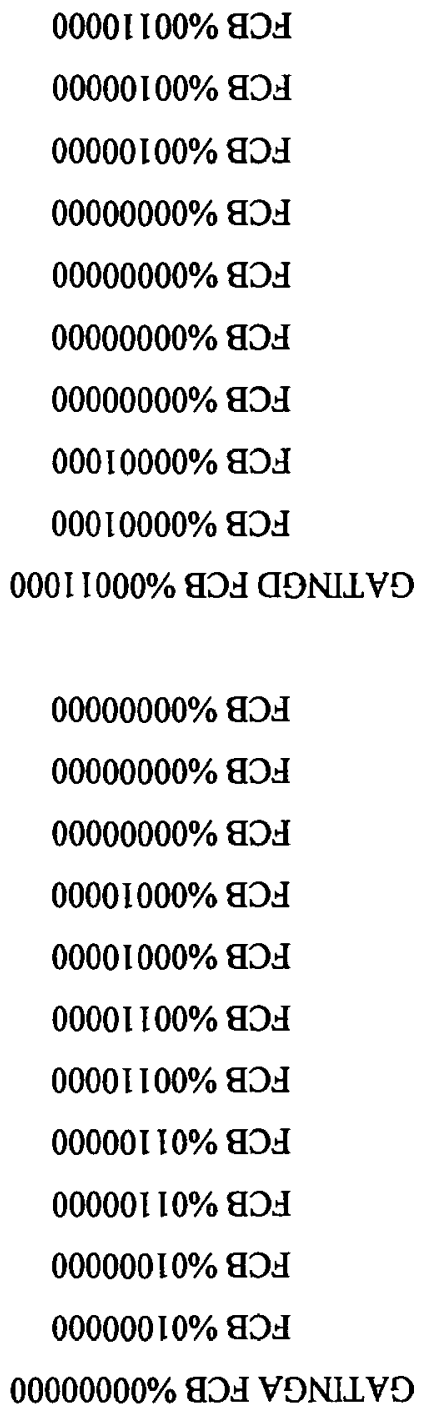


FCB \%001 10000

FCB $\% 00011000$

\section{PATT1 FCB 0}

NOCl FCB 0

;messages to be displayed on PC monitor (CRT) or LCD

CRTMSG1 FCC 'DELAY ANGLE:'

FCB $\$ 0 A$

;line feed

FCB $\$ 04 \quad$;string terminator

CRTMSG2 FCC 'DELAY ANGLE RANGE: 1 TO 179'

FCB $\$ 0 A$

FCB $\$ 04$

LCDMSG1 FCC 'DELAY ANGLE:' ;LCD message

ENDMSG FCB $\$ 04$

;reserve RAM locations

KB_ALPHA RMB 3 ;storage memory for delay angle from keyboard

NUM1 RMB 1 ;number of digits for delay angle from keyboard

BUFFER RMB 3

ALPHA_D FCB 10 ; delay angle ALPHA in degrees

ALPHA FCB 920 ;delay angle ALPHA in \# of counts

D30 EQU $2778 ; 30$ degree in \# of counts

-MAIN PROGRAM

;program initialization

; ORG \$OOE8 ;ICl interrupt vector

; JMP INTER6

; $\quad$ ORG $\$ 00 E 5 \quad$;IC2 interrupt vector

; JMP INTER7 


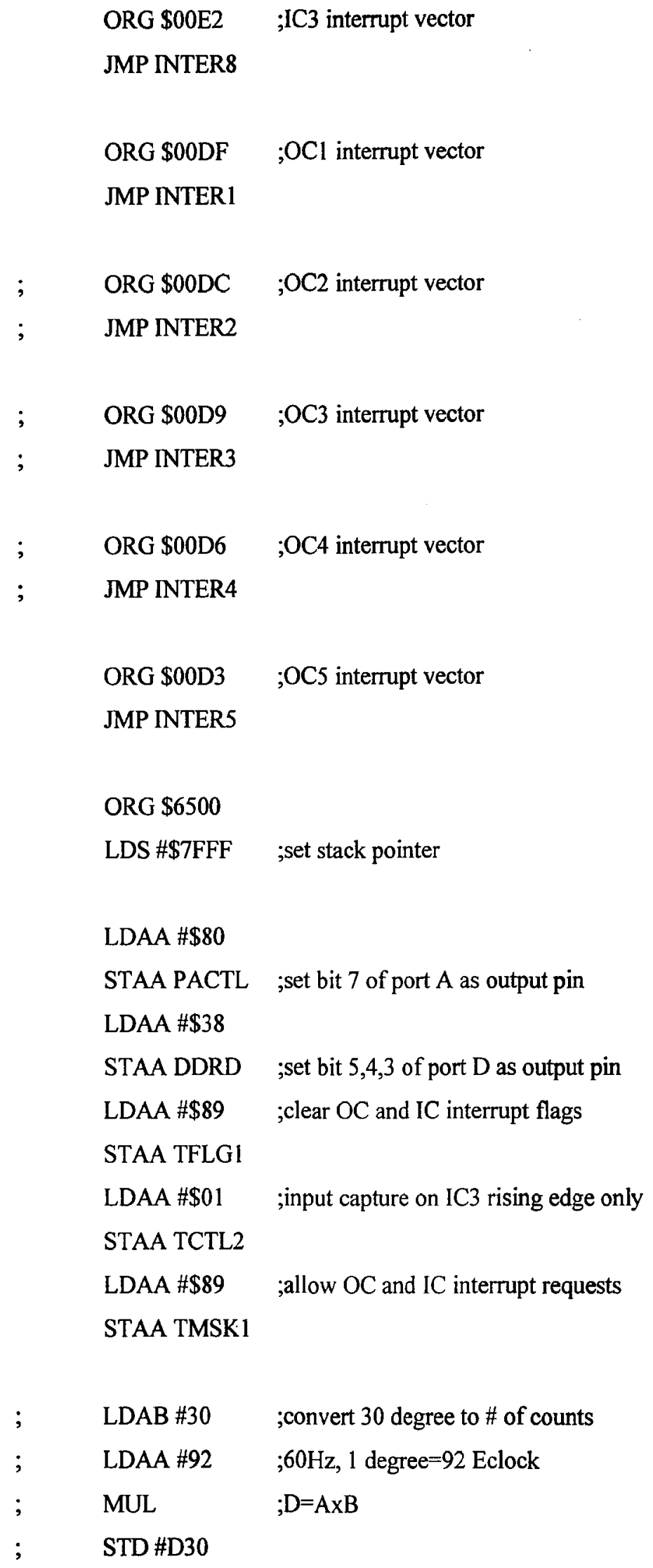




$$
\text { CLI ;interrupt enable }
$$

MAIN LDX \#CRTMSG1 ;display message on PC monitor (CRT)

LDAA $0, X$

JSR OUTSTR

JSR GETCHAR ; get strings from keyboard

JSR LCD_DSP ; display duty cycle on LCD

JSR CONV ; data conversion

JMP MAIN

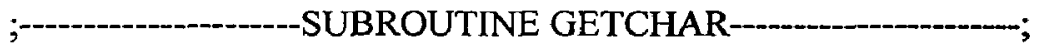

;get characters from keyboard

GETCHAR LDAB \#0

STAB NUM1

LDY \#KB_ALPHA

START JSR INCHAR

LDAB NUM1

CMPB \#0

BEQ NEXT

CMPA \#\$OD

BEQ CHECK 1

;make sure the inputted value is correct

NEXT CMPA \#\$39

BHI ERROR

CMPA \#\$30

BLO ERROR

STAA $0, Y$

LDAB NUM1

INCB

CMPB \#4 
BHS ERROR

STAB NUM1

INY

BRA START

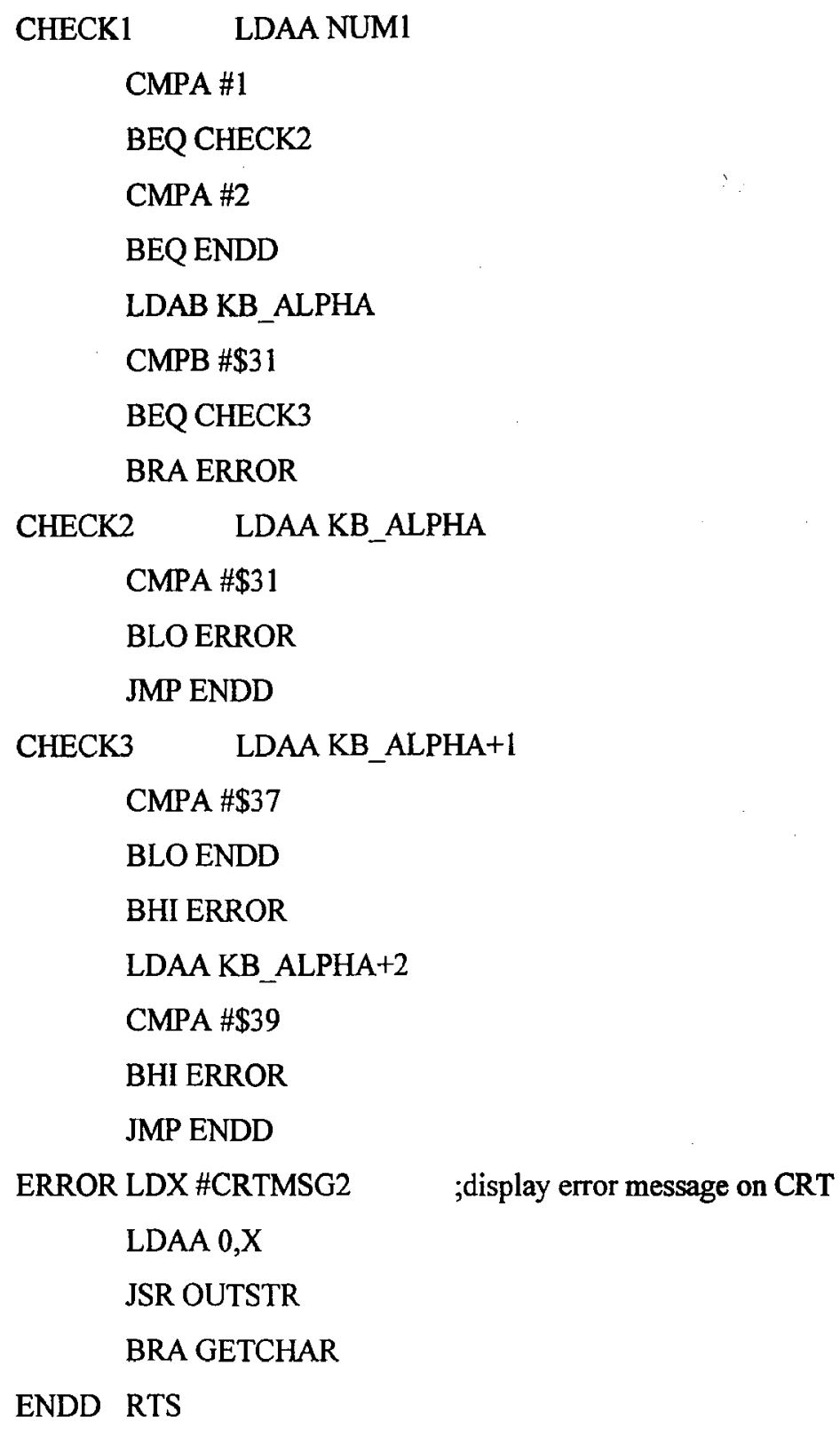


JSR WCTRL

LDAA \#\$38

JSR WCTRL

LDAA \#\$0C

JSR WCTRL

LDY \#LCDMSG1

LOOP2 CPY \#ENDMSG ; compare index register Y,

BEQ NEXT5 ; display a message on LCD one character by

LDAA $0, Y \quad$;another until the end of the message.

JSR WDAT

INY

LDAA \#\$06

JSR WCTRL

BRA LOOP2

NEXT5 CLR BUFFER ; display duty cycle on LCD

LDY \#KB_ALPHA

LOOP1 LDAA 0,Y

JSR WDAT

INY

INC BUFFER

LDAA BUFFER

CMPA NUM1 ;check if all the digits are displayed

BEQ NEXT6

LDAA \#\$06

JSR WCTRL

BRA LOOP1

NEXT6 LDAA \#'D' ; display 'D'

JSR WDAT

RTS

;convert delay angle in hexadecimal to decimal and then number of

;E-clock cycles 


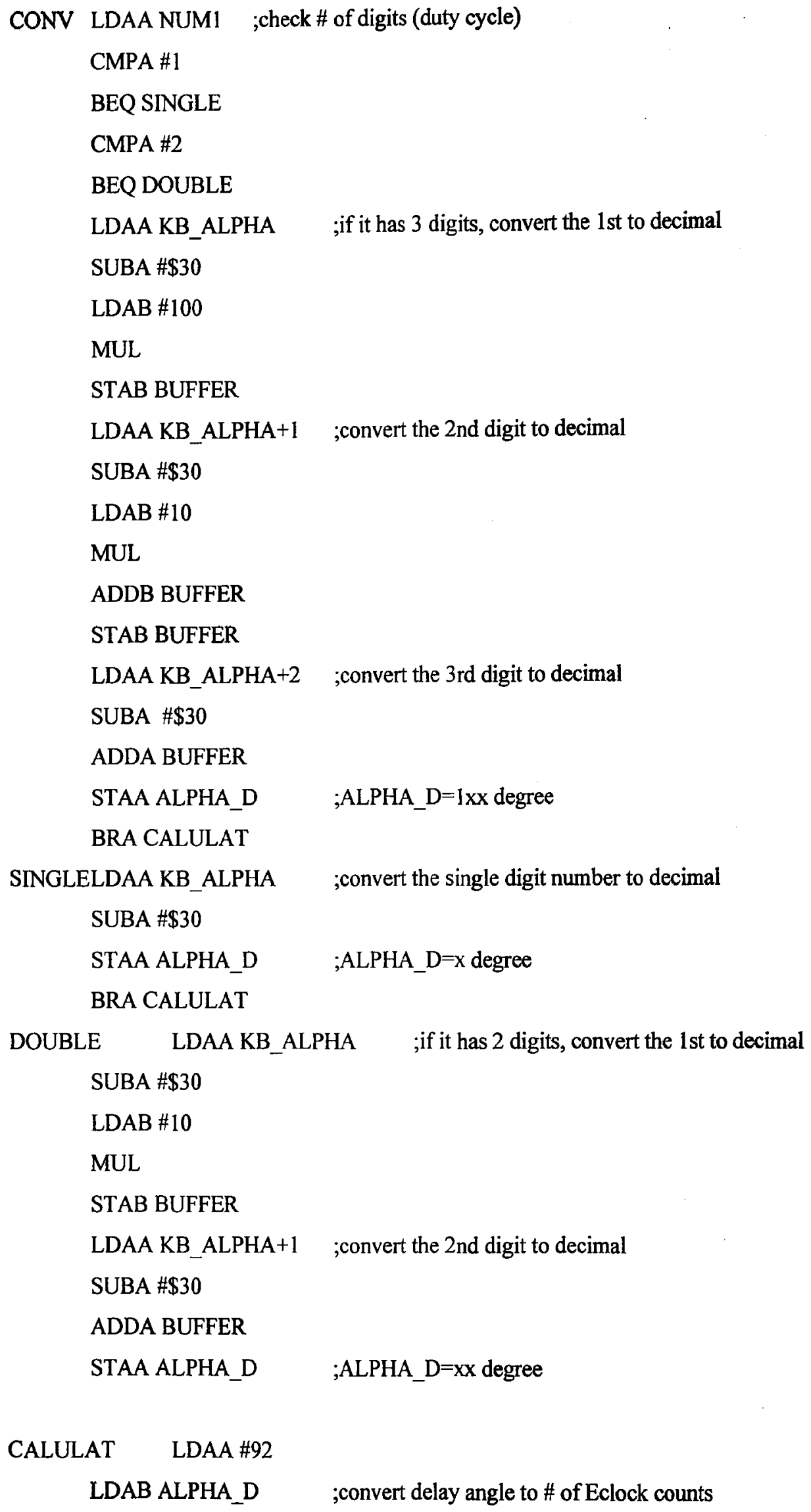


MUL $; \mathrm{D}=\mathrm{AxB}$

STD ALPHA

RTS

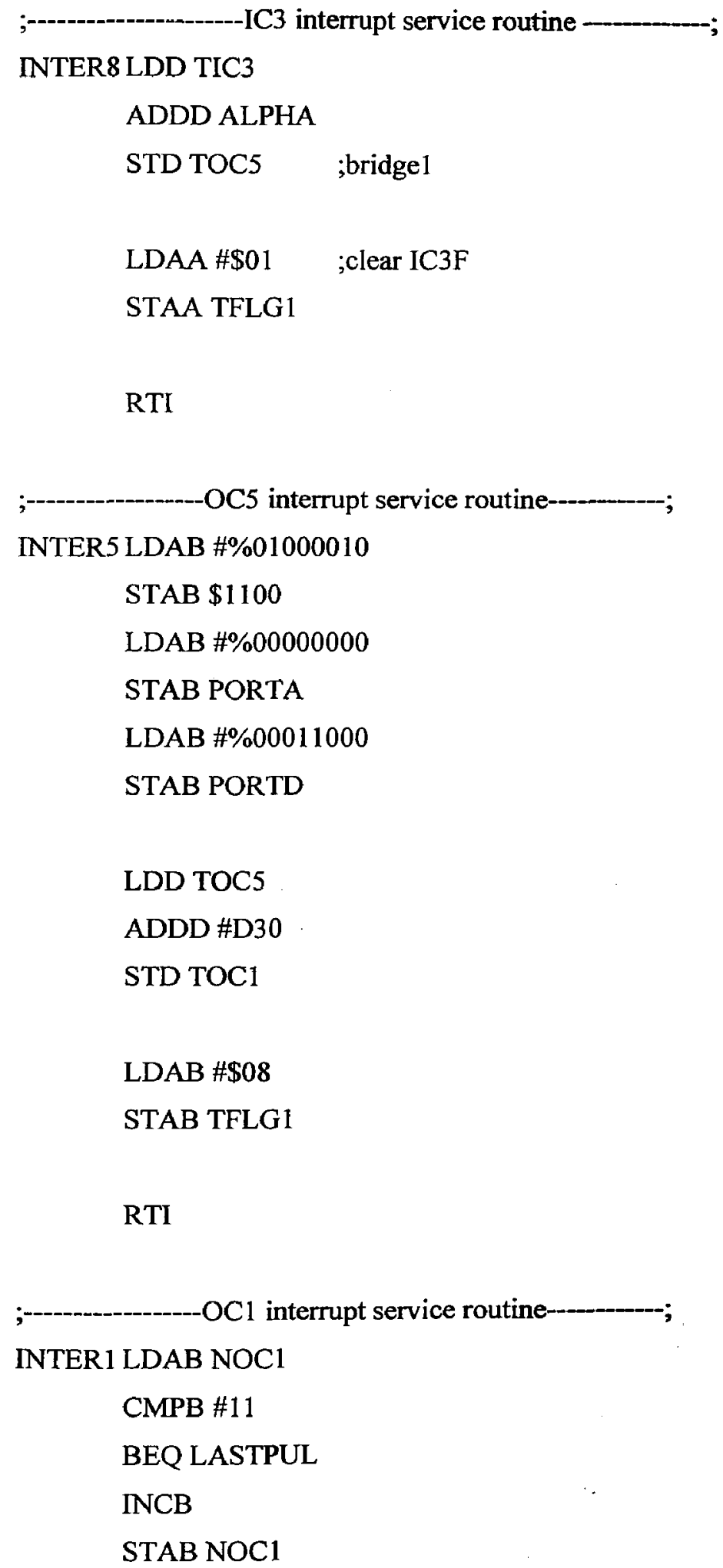


LDAB PATT1

INCB

LDY \#GATINGB

ABY

LDAA 0, Y

STAA $\$ 1100$

LDY \#GATINGA

ABY

LDAA $0, Y$

STAA PORTA

LDY \#GATINGD

ABY

LDAA $0, Y$

STAA PORTD

CMPB \#10

BLS NEXT1

LDAB \#0

NEXT1 STAB PATT1

LDD TOC1

ADDD \#D30

STD TOCI

BRA QUIT

$\begin{array}{ll}\text { LASTPUL LDAB \#0 } & \text { LTAB NOC1 } \\ & \\ \text { QUIT } & \text { LDAB \#\$80 } \\ & \text { STAB TFLG1 } \\ & \text { RTI }\end{array}$




\section{Appendix B THD and PF Curves of the Six- and Multi-pulse SCR Rectifiers}
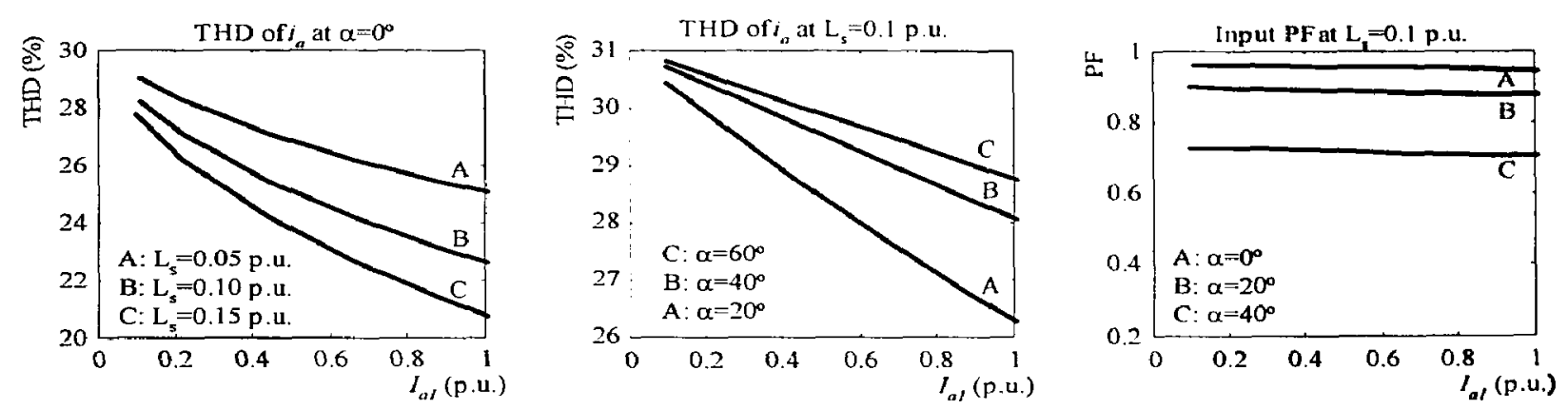

six-pulse with inductive loads
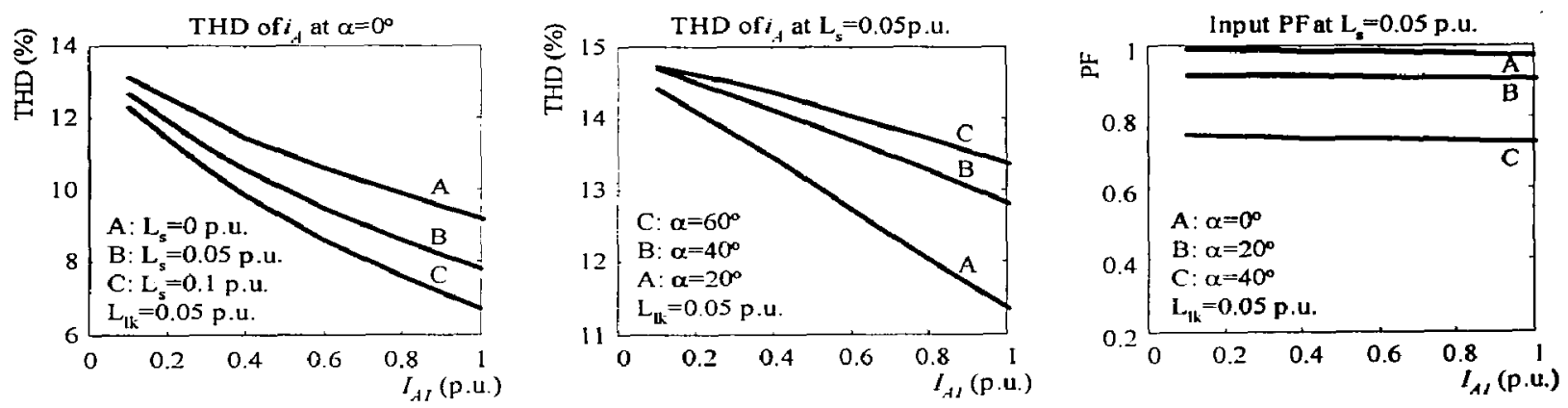

12-pulse with inductive loads
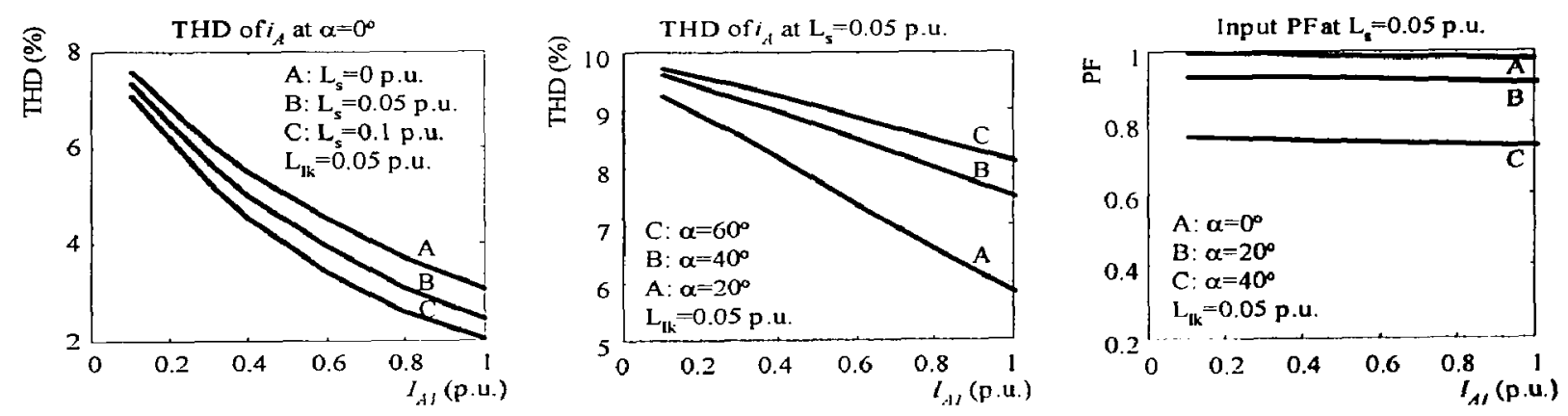

18-pulse with inductive loads
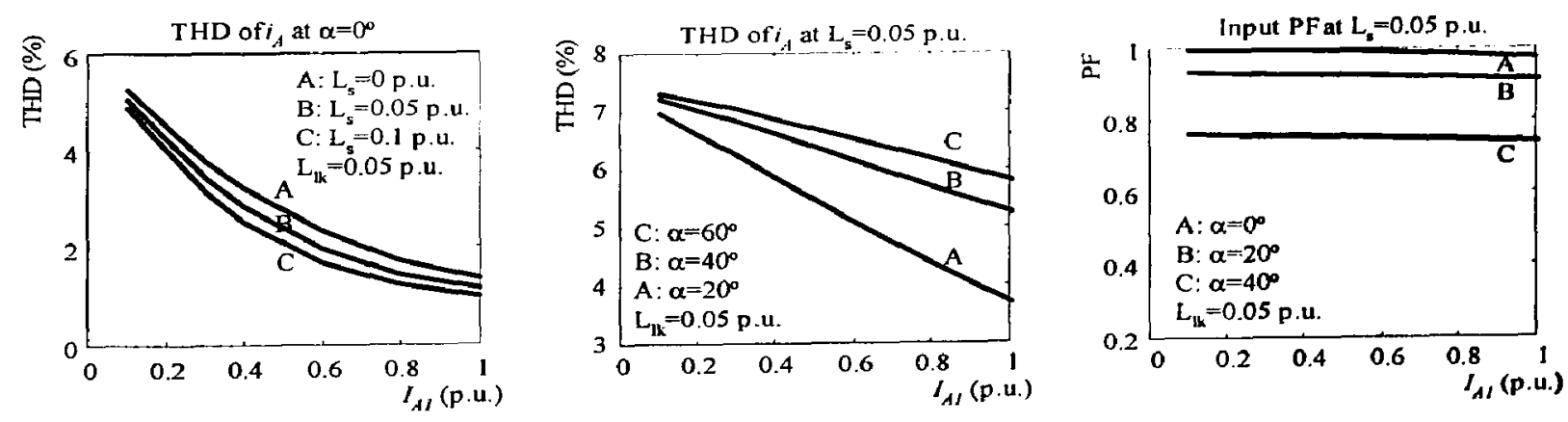

24-pulse with inductive bads 

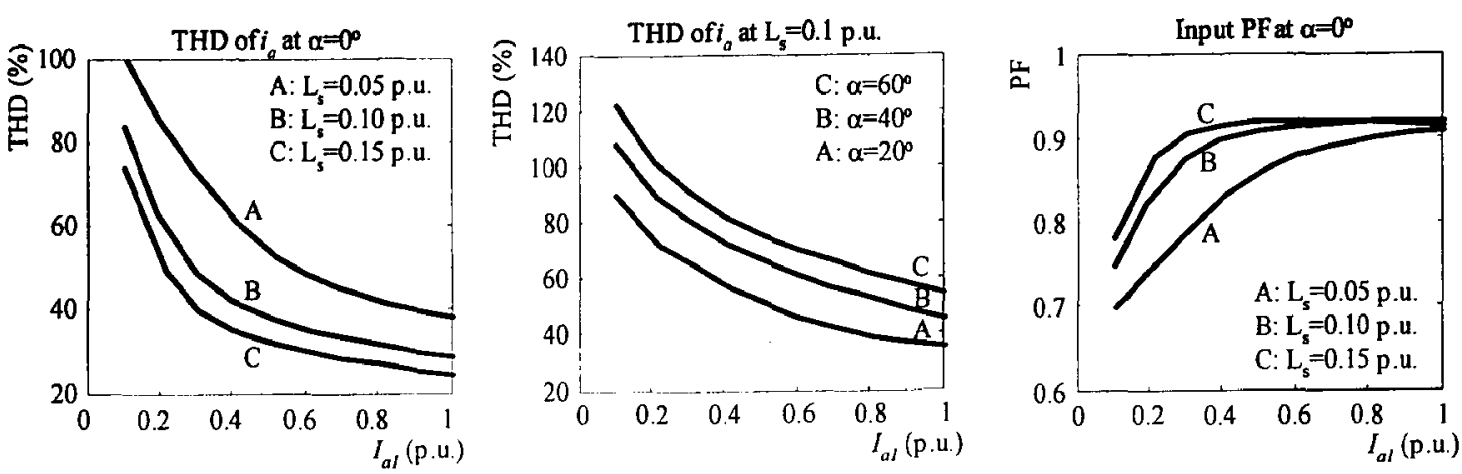

six-pulse with capacitive bads
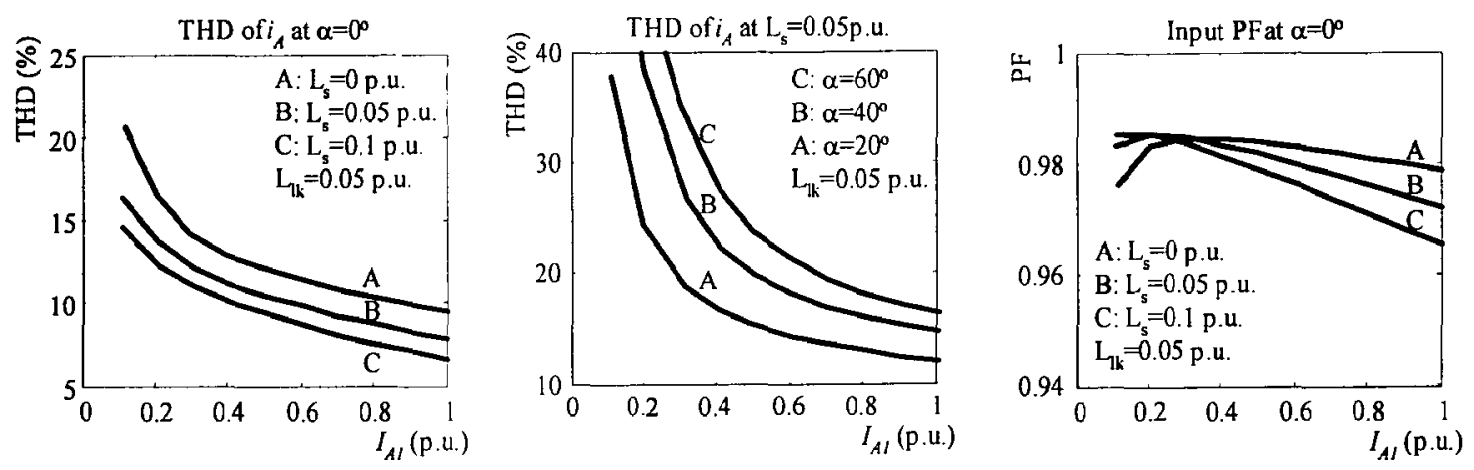

12-pulse with capacitive loads
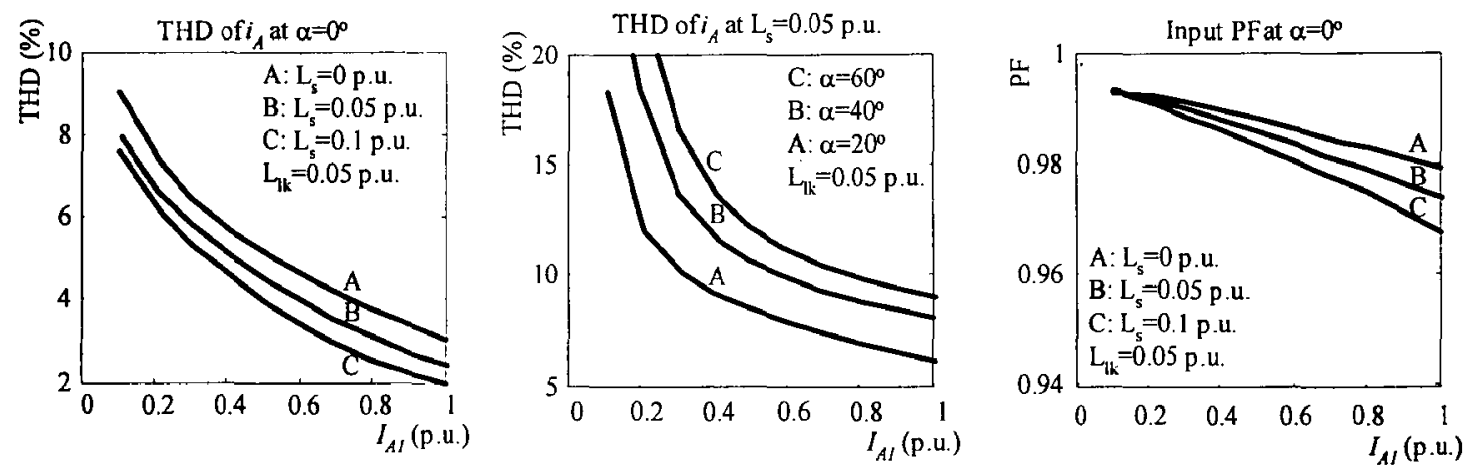

18-pulse with capacitive loads
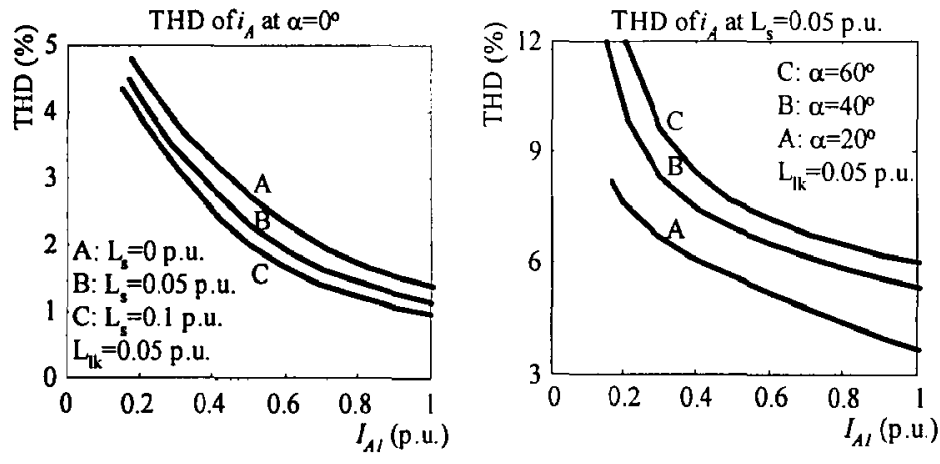

24-pulse with capacitive bads

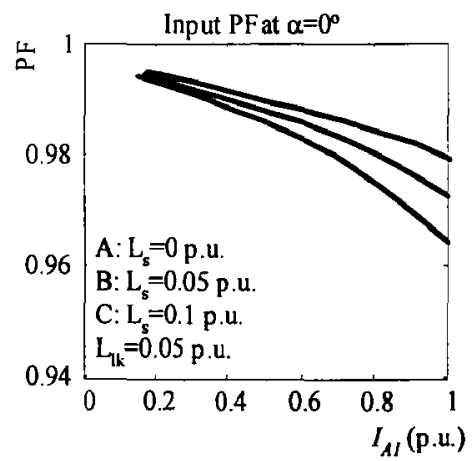




\section{References}

[1] "IEEE recommended practices and requirements for harmonic control in electrical power systems", IEEE Standard 519-1992, pp 78,22,53.

[2] D. A. Paice, "Power electronic converter harmonics: multipulse methods for clean power", Piscataway, NJ : IEEE Press, c1996, pp25-30,137-141.

[3] M. U. Robbins, "Power electronics - converters, applications and design", 2nd Edition, New York : Wiley, c 1995, pp460-463.

[4] M. M. Morcos, C. R. Mersman, N.G. Dillman, "Battery chargers for electric vehicles", IEEE Power Engineering Review, vol.20, Nov. 2000, pp8-11,18.

[5] R. Arseneau, G. T. Heydt, M.J. Kempker, "Application of IEEE Standard 5191992 harmonic limits for revenue billing meters", IEEE Transactions on Power Delivery, vol. 12, no.1, Jan. 1997, pp346-353.

[6] M. M. Swamy, S. L. Rossiter, M. C. Spencer, M. Richardson, "Case studies on mitigating harmonics in ASD systems to meet IEEE519-1992 Standards", IEEE Transactions on Industry Applications, vol. 1, Oct. 1994, pp685-692.

[7] T. Hoevenaars, K. LeDoux, M. Colosino, "Interpreting IEEE Std 519 and meeting its harmonic limits in VFD applications", IEEE Transactions on Industry Applications, Sep. 2003, pp 145-150.

[8] Y. Xiao, B. Wu, F. A. DeWinter, R. Sotudeh, " A dual GTO current-source converter topology with sinusoidal inputs for high-power applications", IEEE Trans. Ind. Applicat., vol. 34, no.4, Jul./Aug. 1998, pp878-884.

[9] R. E. Beighley, C. A. Gougler, J. R. Johnson, "Application of active harmonic filters for power quality improvement", IEEE Power Engineering Society Summer Meeting, vol. 2, Jul. 1999, pp1175-1180.

[10] P. W. Hammond, "A new approach to enhance power quality for medium voltage drives", IEEE Transactions on Industry Applications, Sep. 1995, pp231235. 
[11] "SmartWave switching amplifier - operation manual", Elgar electronics corporation, 2002, pp3-5. 